\title{
Limites Tiltings e Cotiltings
}

\author{
Clezio Aparecido Braga
}

\author{
TESE APRESENTADA \\ $A O$ \\ INSTITUTO DE MATEMÁTICA E ESTATÍSTICA \\ DA \\ UNIVERSIDADE DE SÃO PAULO \\ PARA \\ OBTENÇÃO DO GRAU DE DOUTOR \\ EM \\ MATEMÁTICA
}

\author{
Área de Concentração: Álgebra \\ Orientador: Prof. Dr. Flávio Ulhoa Coelho
}

Durante a elaboração deste trabalho o autor recebeu apoio financeiro da Fapesp de 03/2000 a 06/2002 e dispensa parcial das atividades docentes pela Unioeste. 


\section{Limites Tiltings e Cotiltings}

Este exemplar corresponde à redação final da tese devidamente corrigida, defendida por Clezio Aparecido Braga e aprovada pela comissão julgadora.

São Paulo, 03 de junho de 2005.

Banca examinadora:

- Prof. Dr. Flávio Ulhoa Coelho - Universidade de São Paulo - USP

- Prof. Dr. Eduardo do Nascimento Marcos - Universidade de São Paulo - USP

- Profa. Dra. Sonia Elisabet Trepode - Universidade de Mar del Plata - UMDP

- Prof. Dr. Miguel Angel Alberto Ferrero - Universidade Federal do Rio Grande do Sul - UFRGS

- Prof. Dr. Plamen Eminov Kochloukov - Universidade Estadual de Campinas - UNICAMP 


\section{Agradecimentos}

Gostaria de expressar meus sinceros agradecimentos a todas as pessoas que me ajudaram durante todo o processo de elaboração dessa tese.

Agradeço ao professor Flávio Ulhoa Coelho pela ajuda, paciência, pelas correções feitas no texto e pela compreensão nos momentos difíceis que permitiram que esse trabalho fosse de fato realizado.

Agradeço aos meus verdadeiros amigos que expressaram seu companheirismo durante essa caminhada.

A minha família pela compreensão.

Agradeço especialmente à minha esposa Vanessa que teve paciência em esperar enquanto eu estava debruçado sobre esse trabalho que exigiu muita dedicação. Meus agradecimentos também a Fapesp pelo apoio financeiro de abril de 2000 a junho de 2002.

A todos o meu muito obrigado! 


\begin{abstract}
This work is developed in order to present a process to obtain a tilting module infinitely generated from a countable sequence of finite type modules $T_{i}$ with some properties about their orthogonal categories $T_{i}^{\perp}$ and satisfying $\sup \left\{\operatorname{pd} T_{i}\right\} \leq n$. If the algebra satisfies the finitistic dimension conjecture the last condition is always true and, in this case, the conditions over the orthogonal categories $T_{i}^{\perp}$ are enough. When possible we will show the dual results for cotilting modules. However the central approach is to build tilting modules infinitely generated.

This thesis is organized as follows. In the first chapter we introduce the basic notions and other result not so basic, but necessary in the development of the text. The second chapter is devoted to generalize some results for tilting and cotilting modules over the category $\operatorname{Mod} R$ of all modules over a $\operatorname{ring} R$, and also to list some properties about these classes of modules.

We built, in the third chapter, a direct system of tiltings modules of finite type and describe a process to obtain infinitely generated tiltings modules from finite type ones. The fourth and last chapter were developed to show some algebras where we can build infinitely generated tiltings modules and infinitely generated cotiltings modules. We mainly deal in this chapter with tilted algebra and with some othes classes of algebras which have right stable postprojective component in their quivers.
\end{abstract}




\section{Resumo}

Este trabalho apresenta uma processo de obtenção de um módulo tilting não finitamente gerado a partir de uma seqüência enumerável de módulos $n$-tiltings $T_{i}$ de tipo finito com algumas propriedades sobre suas categorias ortogonais $T_{i}^{\perp}$ e com $\sup \left\{\operatorname{pd} T_{i}\right\} \leq n$. Em álgebras onde vale a conjectura da dimensão finitística esta limitação é sempre verificada bastando observar as condições sobre as subcategorias $T_{i}^{\perp}$. Sempre que possível apresentaremos o análogo para módulos cotiltings, entretanto o enfoque central está na construção de módulos tiltings infinitamente gerados. O trabalho está estruturado em quatro capítulos. No primeiro capítulo introduzimos algumas noções básicas necessárias ao texto, no segundo capítulo desenvolvemos e generalizamos alguns resultados sobre classes tiltings para a categoria $\operatorname{Mod} R$ e listamos uma série de propriedades sobre classes de módulos tiltings e cotiltings não finitamente gerados. No terceiro capítulo construímos o sistema direto de módulos tiltings e o processo de obtenção de tiltings infinitos com limite direto desse limite de módulos tiltings com as propriedades citadas acima. No quarto capítulo mostraremos algumas classes de álgebras onde possademos construir tais módulos tiltings e cotiltings. O enfoque central do capítulo são as álgebras inclinadas e álgebras que contenham uma componente pós-projetiva estável à direita em seu quiver de Auslander Reiten. 


\section{Sumário}

Introdução . . . . . . . . . . . . . . . . . . . . . . . . . 4

1 Preliminares $\quad 6$

1.1 Álgebras e módulos . . . . . . . . . . . . . . . . . . . . . . . 6

1.2 Categorias e funtores . . . . . . . . . . . . . . . . . 8

1.3 Classes de módulos . . . . . . . . . . . . . . . . . . . . . . . 11

1.4 Teoria de cotorção e aproximações . . . . . . . . . . . . . . . . . . 13

1.5 Limites diretos e Extensões . . . . . . . . . . . . . . . . . . . . . . . . 15

1.6 Teoria de Auslander Reiten . . . . . . . . . . . . . . . . . . 23

2 Teoria Tilting Infinitamente Gerada 28

2.1 Definições e resultados iniciais . . . . . . . . . . . . . . . . . . . . . 29

2.2 Teoria tilting e cotilting . . . . . . . . . . . . . . . 34

2.3 Propriedades das classes tiltings e cotiltings . . . . . . . . . . . 39

2.4 Módulos $n$-tiltings parciais de tipo finito . . . . . . . . . . . . 49

2.5 Módulos $n$-tiltings maximais . . . . . . . . . . . . . 50

3 Limites Tiltings e Cotiltings $\quad 53$

3.1 Construção de um sistema direto de módulos tiltings . . . . . . . . . 54

3.2 Limites diretos tiltings e limites inversos cotiltings . . . . . . . . . 56

4 Álgebras com módulos tiltings infinitos $\quad 66$

4.1 Álgebras inclinadas . . . . . . . . . . . . . . . . . . . . 67

4.2 Tilting e cotiltings infinitos em álgebras inclinadas . . . . . . . . . . 70

4.3 Seqüências tiltings . . . . . . . . . . . . . . . . . . . . 79

4.4 Módulos fg-colimites . . . . . . . . . . . . . . . . . . . . . 84

4.5 Outras álgebras com módulos t-colimites . . . . . . . . . . . 88 
Índice Remissivo 


\section{Lista de Figuras}

3.1 Seqüência tilting para uma álgebra hereditária . . . . . . . . . . . . 64

3.2 Seqüência tilting para uma álgebra inclinada . . . . . . . . . . 65

4.1 Seqüência tilting em uma componente de conexão estável à direita 83

4.2 Seqüência cotilting em uma componente de conexão estável à esquerda 83

4.3 Seqüência tilting obtida em uma componente standard generalizada estável à direita . . . . . . . . . . . . . . . . . . . 90 


\section{Introdução}

A teoria tilting é uma teoria bastante explorada e conhecida na teoria de representações de álgebras. Muito se sabe sobre ela atualmente devido aos trabalhos pioneiros de Brenner e Butler ( [17]) e Happel e Ringel ([29]) do inicio da década de 80 , que deram origem à teoria. No início do desenvolvimento da teoria, os módulos tiltings estudados eram todos de dimensão projetiva menor ou igual a 1 . Somente na segunda metade da década de 80, Miyashita ([32]) estendeu a noção de módulos tiltings e cotiltings para módulos de dimensão projetiva finita. Posteriormente, mais precisamente no início dos anos 90, Auslander e Reiten associaram a teoria tilting com a teoria de aproximações ([12]) e estabeleceram relações entre módulos tiltings e classes covariantemente finitas de módulos finitamente gerados.

Vários resultados importantes da teoria tilting e cotilting clássica ainda permanecem válidos quando passamos para contexto dos módulos não finitamente gerados sobre anéis arbitrários. Os primeiros resultados nessa direção foram mostrado por Colpi e Trlifaj ([22]) para módulos de dimensão projetiva menor ou igual a 1 (veja também [11]). A noção de módulo tilting foi então generalizada para dimensão projetiva finita para anéis arbitrários por Angeleri-Hügel e Coelho no início desse século. Apoiados no Teorema de existência de pré-envolventes ([25], Teorema 10), Angeleri-Hügel e Coelho restabeleceram a conexão existente entre as teorias tilting e de aproximações, agora no contexto dos módulos infinitamente gerados. Também conseguiram garantir a existência de complementos para qualquer módulo tilting parcial finitamente gerado, generalizando o resultado de Bongartz ([16]), embora o módulo tilting obtido possa não ser finitamente gerado.

Baseando-se em um exemplo de um módulo tilting infinito exibido por AngeleriHügel e Coelho em [6], obtido como um coproduto de um módulo tilting finitamente gerado, Buan e Solberg construíram um exemplo dual para módulos cotiltings como limite inverso de um sistema inverso de módulos cotiltings. Partindo dessa idéia, e lançando mão das ferramentas das teorias de cotorção e de aproximações e do teorema de existência de pré-envolventes, construímos um módulo tilting como limite direto de um sistema direto de módulos tiltings de tipo finito satisfazando as propriedades duais àquelas utilizadas por Buan e Solberg em [19]. Entretanto, não foi possível manter a mesma limitação da dimensão projetiva da seqüência para o módulo limite.

Embora estejamos trabalhando com um resultado dual ao trabalhado por Buan e Solberg a técnica de demonstração é completamente diferente à por eles utilizada. A idéia central da demonstração é construir a partir de uma seqüência de módulos tiltings uma nova seqüência formando um sistema direto de modo que cada termo da seqüência seja uma pré-envolvente especial do anterior. Ao calcular o colimite 
desse sistema temos um novo módulo tilting não finitamente gerado.

No capítulo 2 do texto generalizaremos alguns resultados sobre a teoria tilting que, até então, só eram conhecidos para módulos fintamente gerados e são imprescindíveis para a demonstração do teorema de construção do limite direto tilting 3.2.1. O capítulo 3 é dedicado à construção do módulo tilting infinitamente gerado. Esse capítulo é o núcleo desse trabalho e destacamos o nosso principal resultado o teorema 3.2.1 sobre o qual esta baseado o restante do texto.

Após termos estabelecido um processo de construção de módulos tiltings não finitamente gerados e estudado a construção de módulos não finitamente gerados, apresentaremos no Capítulo 4 algumas classes de álgebras onde é possível construir esses tipos de módulos. Estudaremos os módulos tiltings infinitamente gerados sobre álgebras hereditárias de tipo de representação infinito, álgebras inclinadas com uma componente de conexão estável à direita e álgebras cujos quivers de Auslander Reiten contenham uma componente pós-projetiva standard generalizada estável à direita e contendo uma seção finita, Teorema 4.5.3. Quando possível, versões duais para módulos cotiltings serão apresentadas.

Vamos explorar a estrutura da componente de conexão de uma $\mathbb{K}$-álgebra inclinada de dimensão finita, gerando uma seqüência tilting com os transladados de Auslander Reiten de um módulo tilting $T_{0}$ fixado. Esse módulo será a soma direta dos módulos sobre um slice na componente de conexão. Além disso, vamos verificar que sobre uma componente de conexão estável à direita, qualquer seqüência tilting crescente (ver definição no Capítulo 4) gera um sistema direto cujo limite direto é um módulo tilting e todos os limites obtidos dessa forma têm a mesma categoria Add. 


\section{Capítulo 1}

\section{Preliminares}

Este primeiro capítulo foi desenvolvido para apresentar a teoria básica necessária à leitura do texto e facilitar a busca dos resultados que serão utilizados no decorrer do da leitura. Os resultados apresentados aqui são em geral resultados clássicos e podem ser encontrados em [1],[13],[24],[25],[36], além disso, os resultados mais importantes, e mesmo alguns resultados clássicos que serão muito utilizados nos capítulos seguintes, serão apresentados com demonstrações e com algumas referências para facilitar a leitura.

No decorrer dos capítulos, exceto afirmação em contrário, todos os anéis considerados são anéis com identidade e sempre que falarmos em módulos estamos considerando módulos à esquerda.

Corolário 1.5.9 é uma das contribuições desse trabalho, mas por ser um resultado relativamente simples e para manter uma coerência no texto o apresentamos neste capítulo.

\section{1 Álgebras e módulos}

Seja $\mathbb{K}$ um corpo. Uma $\mathbb{K}$-álgebra é um anel $A$ (com 1$)$ que possui uma estrutura de $\mathbb{K}$-espaço vetorial de tal forma que $\alpha(a b)=(\alpha a) b=a(\alpha b)$ para todo $a, b \in A$ e $\alpha \in \mathbb{K}$.

Dizemos que $A$ é uma álgebra de dimensão finita se $A$ é um $\mathbb{K}$-espaço de dimensão finita.

Se tivermos, não um corpo $\mathbb{K}$, mas um anel artiniano comutativo $R$, induzindo sobre $A$ uma estrutura de $R$-módulo, de modo que $A$ seja um $R$-módulo finitamente gerado e $\alpha(a b)=(\alpha a) b=a(\alpha b)$ para todo $a, b \in A$ e $\alpha \in R$, dizemos que $A$ é uma 
álgebra de Artin sobre $R$.

Definição 1.1.1 Um quiver $\Delta$ é um grafo orientado. Mais precisamente, um quiver é um par de conjuntos, um conjunto $\Delta_{0}$ de "vértices" e um outro conjunto $\Delta_{1}$ de "flechas" junto com duas funções $s, t: \Delta_{1} \longrightarrow \Delta_{0}$, onde para cada elemento $\alpha \in \Delta_{1}, s(\alpha)$ é o vértice inicial de $\alpha$ e t( $\left.\alpha\right)$ é o vértice final de $\alpha$.

Usaremos a notação $\Delta=\left(\Delta_{0}, \Delta_{1}, s, t\right)$ para representar um quiver.

Definição 1.1.2 Um subquiver de um quiver $\left(\Delta_{0}, \Delta_{1}, s, t\right)$ é um novo quiver $\left(\Delta_{0}^{\prime}, \Delta_{1}^{\prime}, s^{\prime}, t^{\prime}\right)$, tal que $\Delta_{0}^{\prime} \subseteq \Delta_{0}, \Delta_{1}^{\prime} \subseteq \Delta_{1}, t^{\prime}\left(\Delta_{1}^{\prime}\right) \subseteq \Delta_{0}^{\prime}, s^{\prime}\left(\Delta_{1}^{\prime}\right) \subseteq \Delta_{0}^{\prime}$ e, para cada flecha $\alpha \in \Delta_{1}^{\prime}, s^{\prime}(\alpha)=s(\alpha)$ e $t^{\prime}(\alpha)=t(\alpha)$. Se para cada par de vértices $i, j \in \Delta_{0}^{\prime}$, o número de flechas de $i$ para $j$ em $\Delta_{1}^{\prime}$ for o mesmo número de flechas de $i$ para $j$ em $\Delta_{1}$. dizemos que $\left(\Delta_{0}^{\prime}, \Delta_{1}^{\prime}, s^{\prime}, t^{\prime}\right)$ é um subquiver pleno de $\left(\Delta_{0}, \Delta_{1}, s, t\right)$.

Exemplo: Seja $\Delta=\left(\Delta_{0}, \Delta_{1}, s, t\right)$ um quiver onde $\Delta_{0}=\{1,2\}$ e $\Delta_{1}=\{\alpha, \beta\}$ e $s(\alpha)=s(\beta)=1$ e $t(\alpha)=t(\beta)=1$.

Esse quiver pode ser representado pela figura abaixo.

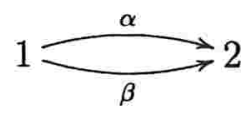

Um caminho "dirigido" de comprimento $n$ de um vértice $i$ para um vértice $j$ em um quiver $\Delta$ é uma sucessão de vértices e flechas $\left(i\left|\alpha_{1} \alpha_{2} \alpha_{3} \ldots \alpha_{n}\right| j\right)$ com $n \geq 0$ verificando as condições:

1. $s\left(\alpha_{1}\right)=i$

2. $t\left(\alpha_{k}\right)=s\left(\alpha_{k+1}\right)$ para todo $1 \leq k \leq n$.

3. $t\left(\alpha_{n}\right)=j$

Se $n=0$ então $i=j$ e temos um caminho trivial associado a cada vértice $i \in \Delta_{0}$. Um quiver é finito se o conjunto de vértice $\Delta_{0}$ e o conjunto de flechas $\Delta_{1}$ são finitos. Dizemos que um quiver $\left(\Delta_{0}, \Delta_{1}, s, t\right)$ é conexo se seu grafo subjacente for conexo e é acíclico se não existir um caminho de comprimento maior ou igual a 1 iniciando e terminando em um mesmo vértice $i$. 
Definição 1.1.3 Dado um corpo $\mathbb{K}$ e um quiver $\Delta$, denote por $\mathbb{K} \Delta$ o $\mathbb{K}$-espaço vetorial gerado por todos os caminhos dirigidos do quiver $\Delta$. Daremos a $\mathbb{K} \Delta$ uma estrutura de $\mathbb{K}$-álgebra da seguinte forma:

1) $S e\left(l\left|\beta_{1} \beta_{2} \ldots \beta_{m}\right| h\right) e\left(i\left|\alpha_{1} \alpha_{2} \ldots \alpha_{n}\right| j\right)$ são dois caminhos dirigidos em $\Delta$ então

$$
\left(i\left|\alpha_{1} \alpha_{2} \ldots \alpha_{n}\right| j\right)\left(l\left|\beta_{1} \beta_{2} \ldots \beta_{m}\right| h\right)=\left\{\begin{array}{l}
0 \quad \text { se } j \neq l \\
\left(i\left|\alpha_{1} \alpha_{2} \ldots \alpha_{n} \beta_{1} \beta_{2} \ldots \beta_{m}\right| h\right) \quad \text { se } j=l
\end{array}\right.
$$

2) Estendemos por linearidade a operação de produto para todos os elementos do $\mathbb{K}$-espaços vetorial $\mathbb{K} \Delta$.

Com a definição acima, $\mathbb{K} \Delta$ torna-se uma $\mathbb{K}$-álgebra, a qual chamaremos de álgebra de caminhos (do quiver $\Delta$ ) (Veja [4]).

Observação: Se $\mathbb{K}$ for um corpo algebricamente fechado, então toda $\mathbb{K}$-álgebra básica de dimensão finita pode ser realizada como um quociente de uma álgebra de caminhos por um ideal "admissível" ([28]).

\subsection{Categorias e funtores}

Seja $\mathcal{C}$ uma classe de elementos quaisquer e para cada par de elementos $A, B \in \mathcal{C}$ seja $\operatorname{Hom}_{\mathcal{C}}(A, B)$ um conjunto: chamaremos os elementos de $\operatorname{Hom}_{\mathcal{C}}(A, B)$ de morfismos e escreveremos $f: A \longrightarrow B$ para os quais $A$ será chamado de domínio e $B$ de contradomínio. Suponha que para cada tripla de elementos $A, B$ e $C$ em $\mathcal{C}$ existe uma função

$$
\circ: \operatorname{Hom}_{\mathcal{C}}(B, C) \times \operatorname{Hom}_{\mathcal{C}}(A, B) \longrightarrow \operatorname{Hom}_{\mathcal{C}}(A, C) .
$$

O elemento de $\operatorname{Hom}_{\mathcal{C}}(A, C)$ imagem do par $(f, g) \in \operatorname{Hom}_{\mathcal{C}}(B, C) \times \operatorname{Hom}_{\mathcal{C}}(A, B)$ pela função "o" será denotado por $f \circ g$ e usaremos $\operatorname{Hom}_{\mathcal{C}}$ para denotar $\bigcup_{A, B \in \mathcal{C}} \operatorname{Hom}_{\mathcal{C}}(A, B)$.

O sistema $\mathfrak{C}=\left(\mathcal{C}, \operatorname{Hom}_{\mathcal{C}}, 0\right)$ é uma categoria se satisfaz:

$\left(\mathrm{C}_{1}\right)$ Para cada tripla $h: C \longrightarrow D, g: B \longrightarrow C$ e $f: A \longrightarrow B$,

$$
h \circ(g \circ f)=(h \circ g) \circ f \text {. }
$$

$\left(\mathrm{C}_{2}\right)$ Para cada $A \in \mathcal{C}$, existe uma única $1_{A} \in \operatorname{Hom}_{\mathcal{C}}(A, A)$ tal que se $f: A \longrightarrow B$ e $g: C \longrightarrow A$, então

$$
f \circ 1_{A}=f \text { e } 1_{A} \circ g=g .
$$


Se $\mathfrak{C}$ for uma categoria, então os elementos da classe $\mathcal{C}$ serão chamados de objetos, a função "o" de composição e os morfismos $1_{A}$ de identidades da categoria.

Um morfismo $f: A \longrightarrow B$ em uma categoria $\mathfrak{C}$ é chamado de isomorfismo quando existe pelo menos um morfismo $f^{-1}: B \longrightarrow A$ tal que $f^{-1} \circ f=1_{A}$ e $f \circ f^{-1}=1_{B}$.

Uma subcategoria de $\mathfrak{C}$ é uma subclasse $\mathcal{C}^{\prime}$ de objetos de $\mathcal{C}$ tal que

$$
\operatorname{Hom}_{\mathcal{C}^{\prime}}(A, B) \subseteq \operatorname{Hom}_{\mathcal{C}}(A, B),
$$

para todo $A \in \mathcal{C}^{\prime}, 1_{A} \in \operatorname{Hom}_{\mathcal{C}^{\prime}}(A, A)$ e $\mathcal{C}^{\prime}$ é fechada para a função composição "o".

Uma subcategoria $\mathcal{C}^{\prime}$ de $\mathcal{C}$ é uma subcategoria plena se

$$
\operatorname{Hom}_{\mathcal{C}^{\prime}}(A, B)=\operatorname{Hom}_{\mathcal{C}}(A, B)
$$

para qualquer $A, B \in \mathcal{C}^{\prime}$.

Um funtor entre duas categorias é uma espécie de "homomorfismo de categorias". Sejam $\mathfrak{C}=\left(\mathcal{C}, \operatorname{Hom}_{\mathcal{C}}, \circ\right)$ e $\mathfrak{D}=\left(\mathcal{D}, \operatorname{Hom}_{\mathcal{D}}, 0\right)$ duas categorias. Um par de funções $\left(F^{\prime}, F^{\prime \prime}\right)$ é um funtor covariante de $\mathfrak{C}$ em $\mathfrak{D}$ se $F^{\prime}$ é uma função de $\mathcal{C}$ em $\mathcal{D}$ e $F^{\prime \prime}$ é uma função de $\operatorname{Hom}_{C}$ em $\operatorname{Hom}_{D}$ tais que para quaisquer $A, B, C \in \mathcal{C}$ e para toda $f: A \longrightarrow B$ e $g: B \longrightarrow C$ em $\mathcal{C}$,

$\mathbf{F}_{1} F^{\prime \prime}(f): F^{\prime}(A) \longrightarrow F^{\prime}(B)$ em $\mathfrak{D}$.

$\mathbf{F}_{2} F^{\prime \prime}(g \circ f)=F^{\prime \prime}(g) \circ F^{\prime \prime}(f)$.

$\mathbf{F}_{3} F^{\prime \prime}\left(1_{A}\right)=1_{F^{\prime}(A)}$.

Um funtor contravariante é um par $\left(F^{\prime}, F^{\prime \prime}\right)$ satisfazendo:

$\mathbf{F}_{1}^{*} F^{\prime \prime}(f): F^{\prime}(B) \longrightarrow F^{\prime}(A)$ em $\mathfrak{D}$.

$\mathbf{F}_{2}^{*} F^{\prime \prime}(g \circ f)=F^{\prime \prime}(f) \circ F^{\prime \prime}(g)$.

$\mathbf{F}_{3}^{*} F^{\prime \prime}\left(1_{A}\right)=1_{F^{\prime}(A)}$.

Se $F, G: \mathfrak{C} \longrightarrow \mathfrak{D}$ são dois funtores, então uma transformação natural de $F$ para $G$ é uma função $\sigma: \mathcal{C} \longrightarrow \operatorname{Hom}_{\mathfrak{D}}$ que associa a cada objeto $A \in \mathcal{C}$ um morfismo $\sigma(A): F(A) \longrightarrow G(A)$ tal que para qualquer morfismo $f \in \operatorname{Hom}_{\mathcal{C}}(A, B)$ existe um diagrama comutativo

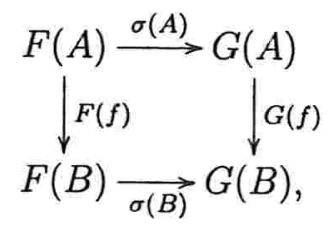


isto é, $\sigma(B) F(f)=G(f) \sigma(A)$.

Dois funtores $F$ e $G$ são isomorfos se existem transformações naturais $\sigma: F \rightarrow$ $G$ e $\zeta: G \rightarrow F$ tais que $\zeta \sigma=i d_{F}$ e $\sigma \zeta=i d_{G}$, onde $i d_{F}$ é a transformação natural de $F$ em $F$ definida pelas aplicações identidades $i d_{F(A)}(A): F(A) \longrightarrow$ $F(A)$. Chamaremos esse isomorfismo de isomorfismo natural ou isomorfismo funtorial. Os funtores $F$ e $G$ são quase inversos se o funtor composto $G F$ for isomorfo ao funtor $1_{\mathfrak{C}}$ e $F G$ for isomorfo a $1_{\mathfrak{D}}$.

Um funtor covariante $F: \mathfrak{C} \longrightarrow \mathfrak{D}$ é uma equivalência de categorias se existir um funtor $G: \mathfrak{D} \longrightarrow \mathfrak{C}$ necessariamente covariante tal que

$$
G F \cong 1_{\mathfrak{C}} \quad \text { e } \quad F G \cong 1_{\mathfrak{D}}
$$

Se isso ocorre dizemos que as categoria $\mathfrak{C}$ e $\mathfrak{D}$ são categorias equivalentes e que $F$ e $G$ são equivalências quase inversas.

Uma par de funtores contravariantes $D: \mathfrak{C} \longrightarrow \mathfrak{D}$ e $D^{\prime}: \mathfrak{D} \longrightarrow \mathfrak{C}$ é uma dualidade entre as categorias $\mathfrak{C}$ e $\mathfrak{D}$ se

$$
D^{\prime} D \cong 1_{\mathfrak{C}} \quad \text { e } \quad D D^{\prime} \cong 1_{\mathfrak{D}}
$$

Temos vários exemplos de categorias, podemos citar entre as mais conhecidas a categoria Set de todos os conjuntos, a categoria Top dos espaços topológicos, a categoria $\operatorname{Mod} R$ dos módulos sobre um anel $R$, a categoria $\bmod A$ a categoria dos módulo finitamente gerados sobre uma álgebra $A$, etc.

Nesse texto trabalharemos com categorias de módulos sobre um anel ou uma álgebra com unidade. Sugerimos uma leitura de [5] ou [24] para maiores detalhes sobre a teoria de categorias.

Usaremos nesse texto as seguintes notações: $\operatorname{Mod} R$ para denotar a categoria de todos os $R$-módulos à esquerda; $\bmod R$ para a subcategoria de todos os módulos finitamente gerados; ind $R$ para subcategorias dos módulos indecomponíveis finitamente gerados; $\mathrm{FP}_{\infty}(R)$ para denotar a subcategoria de $\bmod R$ constituída pelos módulos finitamente gerados que possuem uma resolução projetiva constituída por módulos finitamente gerados; $\mathrm{FP}_{1}(R)$ à categoria dos módulos finitamente apresentados; $\mathcal{P}$ para denotar a subcategoria de $\operatorname{Mod} R$ dos módulos de dimensão projetiva finita; $\mathcal{I}$ para denotar a subcategoria de $\operatorname{Mod} R$ dos módulos de dimensão injetiva finita; $\mathcal{P}_{n}$ para a subcategoria de $\operatorname{Mod} R$ dos módulos de dimensão projetiva menor ou igual a $n ; \mathcal{I}_{n}$ para a subcategoria de $\operatorname{Mod} R$ dos módulos de dimensão injetiva menor ou igual a $n ; \mathcal{P}^{<\infty}$ e $\mathcal{I}^{<\infty}$ para a subcategoria de $\bmod R$ dos módulos de dimensão projetiva e dimensão injetiva finita respectivamente e por fim $\mathcal{P}_{n}^{<\infty}$ e $\mathcal{I}_{n}^{<\infty}$ para as subcategorias de $\bmod R$ dos módulos de dimensão projetiva menor ou igual a $n$ e dimensão injetiva menor o igual a $n$ respectivamente. 
Também usaremos a noção de caminho em uma categoria de módulos. Um caminho de $X$ para $Y$ em $\operatorname{Mod} R$ é uma sucessão de morfismos

$$
X=X_{1} \rightarrow X_{2} \rightarrow \ldots \rightarrow X_{n}=Y
$$

Para um $R$-módulo $M$, usaremos Add $M$ para a subcategoria de todos os somandos diretos de coprodutos de $M$ e $\operatorname{Prod} M$ para a subcategoria de todos os somandos diretos de produtos de $M$. No caso de $M$ ser finitamente gerado usaremos a notação add $M$ para denotar os somandos diretos de somas finitas de cópias de $M$.

Vejamos a seguir duas categorias obtidas a partir da categoria de módulos.

Seja $A$ uma álgebra de Artin. Denotarmos $\mathcal{P}(X, Y)$ ao subgrupo de $\operatorname{Hom}(X, Y)$ constituído pelos morfismos de $X$ em $Y$ que se fatoram através de um projetivo e $\mathcal{I}(A, B)$ ao subgrupo de $\operatorname{Hom}(A, B)$ formado pelos morfismos que se fatoram através de algum injetivo. Considere os grupos quocientes

$$
\underline{\operatorname{Hom}}(X, Y)=\frac{\operatorname{Hom}(X, Y)}{\mathcal{P}(X, Y)} \text { e } \overline{\operatorname{Hom}}(X, Y)=\frac{\operatorname{Hom}(X, Y)}{\mathcal{I}(X, Y)} .
$$

Defina as categorias $\underline{\bmod } A$, onde os objetos são os mesmos $\operatorname{de} \bmod A$ e como morfismos em

$$
\underline{\operatorname{Hom}}(X, Y)=\frac{\operatorname{Hom}(X, Y)}{\mathcal{P}(X, Y)}
$$

e $\overline{\bmod } A$ como os mesmos objetos $\operatorname{de} \bmod A$ e com morfismos em

$$
\overline{\operatorname{Hom}}(X, Y)=\frac{\operatorname{Hom}(X . Y)}{\mathcal{I}(X, Y)} .
$$

Observe que as translações de Auslander Reiten $\tau: \underline{\bmod } A \rightarrow \overline{\bmod } A$, e $\tau^{-}$: $\overline{\bmod } \rightarrow \underline{\bmod }$ são equivalências quase inversas entre essas categorias. Uma apresentação detalhada sobre a construção dos funtores de Auslander Reiten pode ser encontrada em [13]. No final desse capítulo voltaremos a falar da teoria de Auslander Reiten e então teremos mais detalhes sobre as características do funtores $\tau^{n} \mathrm{e}$ $\tau^{-n}$.

\subsection{Classes de módulos}

Definição 1.3.1 Uma classe de módulos $\mathcal{C}$ é resolvente se:

i) $\mathcal{C}$ contém todos os módulos projetivos. 
ii) $\mathcal{C}$ é fechada para somandos diretos e extensões, $e$

iii) $\mathrm{Se} 0 \longrightarrow X \longrightarrow Y \longrightarrow Z \longrightarrow 0$ é uma seqüência exata com $Y$ e $Z \in \mathcal{C}$, então $X \in \mathcal{C}$

A condição iii) pode ser interpretada dizendo que $\mathcal{C}$ é fechada para núcleos de epimorfismos.

O item iii) é equivalente a: se existe um seqüência exata

$$
0 \longrightarrow X \longrightarrow P \longrightarrow Y \longrightarrow 0
$$

com $P$ um projetivo e $Y \in \mathcal{C}$, então $X \in \mathcal{C}$.

Definição 1.3.2 Para uma classe $\mathcal{C} \subseteq \operatorname{Mod} R$, definimos $\mathcal{C}^{\perp_{1}}=\operatorname{KerExt}^{1}\left(\mathcal{C},{ }_{-}\right)$, $\left.\left.{ }^{\perp_{1}} \mathcal{C}=\operatorname{KerExt}^{1}{ }_{-}, \mathcal{C}\right), \mathcal{C}^{\perp}=\bigcap_{i \geq 1} \operatorname{KerExt}^{i}\left(\mathcal{C},{ }_{-}\right), e^{\perp} \mathcal{C}=\bigcap_{i \geq 1} \operatorname{KerExt}^{i}{ }_{-}, \mathcal{C}\right)$

Um fato importante sobre classes de módulos é que se $\mathcal{C}$ for uma classe qualquer em $\operatorname{Mod} R$, a classe ${ }^{\perp} \mathcal{C}$ de $\operatorname{Mod} R$ satisfaz as condições i), ii) e iii) da definição acima e será, portanto, resolvente. Outro fato bastante importante é que toda classe resolvente de $\bmod R$ está contida em $\mathrm{FP}_{\infty}(R)$, portanto temos que ${ }^{\perp} \mathcal{C} \cap \bmod R \subseteq$ $\mathrm{FP}_{\infty}(R)$ (Veja [8] Lema 1.1).

Definição 1.3.3 Dualmente uma classe de módulos $\mathcal{C}$ é corresolvente se:

i) C contém todos os módulos injetivos;

ii) $\mathcal{C}$ é fechada para somandos diretos e extensões;

iii) $S e 0 \longrightarrow X \longrightarrow Y \longrightarrow Z \longrightarrow 0$ é uma seqüência exata com $X \quad$ e $Y \in \mathcal{C}$, então $Z \in \mathcal{C}$.

A condição iii) pode ser interpretada dizendo que $\mathcal{C}$ é fechada para conúcleos de monomorfismos.

Dualmente temos que iii) é equivalente a: se existe um seqüência exata

$$
0 \longrightarrow X \longrightarrow I \longrightarrow Y \longrightarrow 0
$$


com $I$ um injetivo, e $X \in \mathcal{C}$, então $Y \in \mathcal{C}$.

Por dualidade $\mathcal{C}^{\perp}$ é corresolvente para qualquer classe $\mathcal{C}$.

Sejam $M$ um $R$-módulo e $i \in \mathbb{N}$, denotaremos por $\Omega^{i}$ à classe de todos os $i$-ésimos módulos syzygies ocorrendo em alguma resolução projetiva de $M$ e $\Omega^{0}=\{M\}$. De forma análoga denotaremos a classe de todos os $i$-ésimos módulos cosyzygies que ocorrem em alguma corresolução injetiva de $M$ por $\Omega^{-i}(M)$. Veja maiores detalhes sobre as classes $\Omega^{i}$ s em [13].

Quando $M \in \mathrm{FP}_{\infty}(R)$ ou se $R$ for um anel coerente e $M \in \mathrm{FP}_{1}(R)$, vamos considerar somente resoluções, onde os módulos sygygies pertencem a $\mathrm{FP}_{\infty}(R)$ e $\mathrm{FP}_{1}(R)$ respectivamente.

Seja $\mathcal{C} \subseteq \operatorname{Mod} R$ uma subclasse de módulos. Para um número inteiro $i$ definimos $\Omega^{i}(\mathcal{C})=\bigcup_{M \in \mathcal{C}} \Omega^{i}(M)$

Diremos que uma classe $\mathcal{C}$ é s-fechada se $\Omega^{1}(\mathcal{C}) \subseteq \mathcal{C}$. Analogamente $\mathcal{C}$ é dita cfechada se $\Omega^{-1}(\mathcal{C}) \subseteq \mathcal{C}$. É conseqüência imediata da definição que se $\mathcal{C}$ é s-fechada, então todas as classes $\Omega^{i}(\mathcal{C})$ serão s-fechadas e que se $\mathcal{C}$ é c-fechada, então todas as classes $\Omega^{-i}(\mathcal{C})$ serão c-fechadas.

\subsection{Teoria de cotorção e aproximações}

Introduziremos a seguir o conceito de pré-envolventes e pré-coberturas, também usaremos a noção de classes pré-envolventes e classes de recobrimento (precover class em inglês). Esses conceitos foram introduzidos independentemente por Enochs, para a categoria $\operatorname{Mod} R$ sobre um anel $R$ em [23] e por Auslander e Smalø em [14] e [15] para a categoria $\bmod \Lambda$ para uma álgebra de Artin $\Lambda$. Essas noções foram diretamente motivadas pelo conceito de envolvente injetiva e de modo geral, envolventes injetivas, cobertura projetivas, envolventes injetivas puras, coberturas planas e coberturas livres podem ser interpretadas como casos específicos de préenvolventes e pré-coberturas.

Definição 1.4.1 Seja $\mathcal{C}$ uma classe de módulos.

1. Uma $\mathcal{C}$-pré-envolvente para um $R$-módulo $X$, é um par $(M, f)$ com $M \in \mathcal{C}$ e $f: X \longrightarrow M$, tal que qualquer morfismo $h: X \longrightarrow Y$ com $Y \in \mathcal{C}$ fatorase através de $M$. Equivalentemente, o morfismo de grupos $\operatorname{Hom}(X, M) \stackrel{f_{\bullet}}{\longrightarrow}$ $\operatorname{Hom}(X, Y)$ é sobrejetivo. 
Se além disso $f$ for um monomorfismo $e \operatorname{Coker}(f) \in^{\perp} \mathcal{C}$, então dizemos que $(M, f)$ é uma pré-envolvente especial.

Uma classe $\mathcal{C}$ é uma classe pré-envolvente se todo $R$-módulo possui uma $\mathcal{C}$-pré-envolvente especial.

2. Uma $\mathcal{C}$-pré-cobertura para um $R$-módulo $X$ é um par $(M, g)$ com $M \in \mathcal{C} e$ $g: M \longrightarrow X$, tal que todo morfismo $h: Y \longrightarrow X$ com $Y \in \mathcal{C}$ fatora-se através de $M$. Equivalentemente $\operatorname{Hom}(M, X) \stackrel{f_{*}}{\longrightarrow} \operatorname{Hom}(Y, X)$ é sobrejetivo.

Se além disso $g$ for um epimorfismo e $\operatorname{Ker}(f) \in \mathcal{C}^{\perp}$, então dizemos que $(M, g)$ é uma pré-cobertura especial.

Uma classe $\mathcal{C}$ é uma classe de recobrimento se todo $R$-módulo possui uma $\mathcal{C}$-pré-cobertura especial.

No contexto de álgebras de Artin as terminologias usuais (introduzidas por Auslander e Smalø) para os conceitos de pré-envolventes e pré-coberturas são aproximações à esquerda e aproximações à direita respectivamente.

Um par de classes de módulos $\mathcal{C}=(\mathcal{A}, \mathcal{B})$ é um par de cotorção se $\mathcal{A}={ }^{{ }_{1}} \mathcal{B}$ e $\mathcal{B}=\mathcal{A}^{\perp_{1}}$. Um par de cotorção e dito cogerado por uma classe (ou por um conjunto) $\mathcal{S}$ se $\mathcal{B}=\mathcal{S}^{\perp_{1}}$.

Dizemos que um par de cotorção $\mathcal{C}=(\mathcal{A}, \mathcal{B})$ é completo se: $\mathcal{B}$ é uma classe pré- envolvente e $\mathcal{A}$ é de recobrimento. Esse conceito estabelece uma ligação entre a teoria de cotorção e a teoria de aproximações. A ligação entre essas teorias é um fato relativamente recente e tem origem em Salce [37] e pode ser vista como uma substituta à não existência de dualidades na categoria $\operatorname{Mod} R$, permitindo a dualização de resultados que na categoria de módulos finitamente gerados era conseqüência do funtor de dualidade $D$.

Definição 1.4.2 Uma classe $\mathcal{B}$ em $\operatorname{Mod} R$ é de tipo finito se existe uma classe de módulos $\mathcal{S} \subseteq \mathrm{FP}_{\infty}(R)$ tal que $\mathcal{B}=S^{\perp_{1}}$, isto é, $\mathcal{B}$ é cogerada por uma classe $\mathcal{S} \subseteq \mathrm{FP}_{\infty}(R)$.

Se $\mathcal{S}$ for uma classe s-fechada então $\mathcal{S}^{\perp_{1}}=S^{\perp}$ e como $\mathcal{S}^{\perp}$ é corresolvente fechada para produtos diretos e contém todos os injetivos, ${ }^{\perp_{1}}\left(\mathcal{S}^{\perp}\right)={ }^{\perp}\left(\mathcal{S}^{\perp}\right)$. Portanto o par $\left({ }^{\perp}\left(\mathcal{S}^{\perp}\right), \mathcal{S}^{\perp}\right)$ é um par de cotorção cogerado por $\mathcal{S}$. 
Proposição 1.4.3 ([25] teorema 10) Seja $(\mathcal{A}, \mathcal{B})$ um par de cotorção cogerado por um conjunto de módulos, então $\mathcal{B}$ é uma classe pré-envolvente e $\mathcal{A}$ é uma classe de recobrimento. Em outras palavras $(\mathcal{A}, \mathcal{B})$ é completo.

Ainda é um problema em aberto, quando o dual da Proposição 1.4.3 é válido para qualquer conjunto de módulos. Entretanto, a existência de algumas classes de recobrimento é garantida por [2]. As informações de [2] foram sintetizadas por Angeleri-Hügel e Coelho em [6] e conseguiu-se uma forma dual de 1.4.3 para a classe ${ }^{\perp} M$ quando $M$ for um módulo cotilting. Esse resultado é apresentado pela Proposição 1.4.4 abaixo.

Proposição 1.4.4 Se $M \in \operatorname{Mod} R$ é um módulo cotilting, então todo $R$-módulo possui uma ${ }^{\perp} M$-pré-cobertura.

Como estamos interessados em módulos tiltings e cotiltings as proposições 1.4.3 e 1.4.4 serão suficientes para nosso propósito.

\subsection{Limites diretos e Extensões}

Os resultados que apresentaremos abaixo utilizam-se da noção de limite direto e caracterizam o comportamento desse módulos com relação aos funtores de extensão Ext $^{i}$.

Vamos ver primeiramente a definição de um sistema direto.

Um conjunto $I$ é dito quase ordenado se $I$ é dotado de uma relação binária $\preccurlyeq$ que seja reflexiva e transitiva. Denotaremos um conjunto quase ordenado $I$ por $(I, \preccurlyeq)$.

Um conjunto quase ordenado $(I, \preccurlyeq)$ é dirigido se para cada $i, j \in I$, existir um $k \in I$ tal que $i \preccurlyeq k$ e $j \preccurlyeq k$.

Seja $(I, \preccurlyeq)$ um conjunto quase ordenado. Podemos construir uma categoria $\mathcal{A}$, na qual os objetos são os elementos de $I$. E se $x$ e $y$ são objetos em $\mathcal{A}$, definimos $\operatorname{Hom}(x, y)=\left\{i_{y}^{x}\right\}$, o conjunto unitário contendo o elemento $i_{y}^{x}$ se $x \preccurlyeq y$, e $\operatorname{Hom}(x, y)=\phi$ caso contrário. A composição em $\mathcal{A}$ é dada por $i_{z}^{y} i_{y}^{x}=i_{z}^{x}$, sempre que $x \preccurlyeq y \preccurlyeq z$.

Definição 1.5.1 Sejam $(I, \preccurlyeq)$ um conjunto quase ordenado, $\mathcal{C}$ uma categoria e $\mathcal{A}$ a categoria definida acima. Um sistema direto em $\mathcal{C}$ com índices em I é um funtor 
$F: \mathcal{A} \rightarrow \mathcal{C}$, que para cada objeto $x \in \mathcal{A}$ associa $F(x)=F_{x}$, o qual é um objeto em $\mathcal{C}$, e para cada morfismo $i_{y}^{x} \in \mathcal{A}, F\left(i_{y}^{x}\right)=\varphi_{y}^{x}: F_{x} \rightarrow F_{y}$ é um morfismo em $H_{0} m_{\mathcal{C}}$ satisfazendo:

(a) $\varphi_{x}^{x}: F_{x} \rightarrow F_{x}$ é a identidade para todo $x \in \mathcal{A}$.

(b) Se $x \preccurlyeq y \preccurlyeq z$, o seguinte diagrama comuta

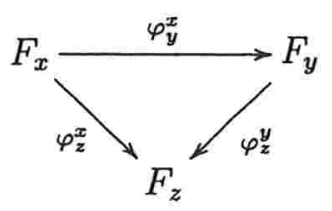

Definição 1.5.2 Seja $F=\left\{F_{i}, \varphi_{j}^{i}\right\}$ um sistema direto em uma categoria $\mathcal{C} . \quad O$ limite direto deste sistema, denotado por $\lim _{\bar{i} \in \mathfrak{I}} F_{i}$, é um objeto em $\mathcal{C}$ e uma familia de morfismos $\alpha_{i}: F_{i} \rightarrow \lim _{\bar{i} \in \vec{I}} F_{i}$ tais que:

(a) $\alpha_{i}=\alpha_{j} \varphi_{j}^{i}$ sempre que $i \preccurlyeq j$.

(b) Para cada objeto $X$ e toda familia de morfismos $f_{i}: F_{i} \rightarrow X$, tal que $f_{i}=f_{j} \varphi_{j}^{i}$ sempre que $i \preccurlyeq j$, existe um único morfismo $\beta: \lim _{i \in i} F_{i} \rightarrow X$ de modo que o diagrama abaixo comuta.

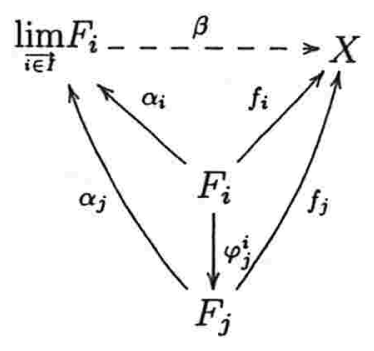

A noção de limite inverso é dual.

No nosso caso trataremos com limites diretos e inversos sobre categorias de módulos, ou seja $\mathcal{C}=\operatorname{Mod} R$ para algum anel $R$, nesse caso simbolizaremos o módulo $F_{i}$ por $X_{i}$. Vale lembrar que o limite direto de um sistema de módulos sempre existe. Veja por exemplo, [36].

Usaremos a noção de limite direto em associação com os funtores de extensão $\operatorname{Ext}(-,-$-). O seguinte lema é uma generalização do caso Noetheriano e é um dos principais resultados que associa limites diretos e homologias ([24] Lema 10.2.4). 
Lema 1.5.3 Sejam $R$ um anel, $M$ um módulo em $\operatorname{FP}_{\infty}(R) e \underset{i \in 1}{\lim } N_{i}$ um limite direto de R-módulos. Então

$$
\operatorname{Ext}_{R}^{j}\left(M, \lim _{i \in I} N_{i}\right) \cong \lim _{i \in \mathbb{I}} \operatorname{Ext}_{R}^{j}\left(M, N_{i}\right) \text { para todo } j>0 .
$$

Prova: Como $M \in \mathrm{FP}_{\infty}$ existe uma resolução projetiva

$$
\cdots \longrightarrow P_{1} \longrightarrow P_{0} \longrightarrow M \longrightarrow 0
$$

constituída de módulos finitamente gerados, então é possível obter uma resolução

$$
\cdots \longrightarrow F_{1} \longrightarrow F_{0} \longrightarrow M \longrightarrow 0
$$

com $F_{i} R$-módulos livres finitamente gerados.

Considere o complexo

$$
0 \longrightarrow \operatorname{Hom}\left(M, \lim _{i \in I} N_{i}\right) \longrightarrow \operatorname{Hom}\left(F_{0}, \lim _{i \in I} N_{i}\right) \longrightarrow \operatorname{Hom}\left(F_{1}, \lim _{i \in I} N_{i}\right) \longrightarrow \cdots,
$$

onde cada $F_{k}=\coprod_{l=1}^{n_{k}} R$. Então

$$
\begin{gathered}
\operatorname{Hom}\left(F_{k}, \lim _{\overrightarrow{i \in I}} N_{i}\right) \cong \operatorname{Hom}\left(\coprod_{l=1}^{n_{k}} R, \lim _{i \in I} N_{i}\right) \cong \coprod_{l=1}^{n_{k}} \lim _{i \in I} N_{i} \cong \\
\coprod_{l=1}^{n_{k}} \lim _{i \in I} \operatorname{Hom}\left(R, N_{i}\right) \cong \lim _{\overrightarrow{i \in I}} \operatorname{Hom}\left(\coprod_{l=1}^{n_{k}} R, N_{i}\right) \cong \lim _{\vec{i} I} \operatorname{Hom}\left(F_{k}, N_{i}\right) \text { para todo } j>0 .
\end{gathered}
$$

Como limite direto comuta com homologia temos que

$$
\operatorname{Ex}^{j}\left(M, \underset{i \in I}{\lim } N_{i}\right) \cong \underset{i \in I}{\lim \operatorname{Ext}^{j}}\left(M, N_{i}\right)
$$

Se $R$ for um anel coerente à esquerda, todo módulo finitamente apresentado está em $\mathrm{FP}_{\infty}(R)$. Lembrando que um anel $R$ é corente à esquerda se todo submódulo à esquerda de um $R$ módulo finitamente apresentado é finitamente apresentado. Em particular Álgebras de Artin são anéis coerentes. Por isso, não há restrição em trabalhar com a subcategoria $\mathrm{FP}_{\infty}(R)$ para essas álgebras.

Corolário 1.5.4 Sejam $R$ um anel coerente, $M$ um módulo em $\operatorname{FP}_{1}(R)$ e $\lim _{i \in I} N_{i} u m$ limite direto de R-módulos. Então 


$$
\operatorname{Ext}_{R}^{j}\left(M, \lim _{i \in 1} N_{i}\right) \cong \lim _{i \in I} \operatorname{Ext}_{R}^{j}\left(M, N_{i}\right) \text { para todo } j>0
$$

Corolário 1.5.5 $\mathrm{Se} \mathcal{C} \subseteq \mathrm{FP}_{\infty}(R)$, então $\mathcal{C}^{\perp}$ é fechada para limites diretos.

Definição 1.5.6 Seja $\mu$ um ordinal. Um sistema $\left.\left\{\left(M_{\alpha}, f_{\alpha \beta}\right) \mid \alpha \leq \mu\right)\right\}$ é um sistema direto contínuo se, $M_{\alpha}=\lim _{\bar{\delta}<\alpha} M_{\delta}$ para todo ordinal limite $\alpha \leq \mu$.

O seguinte lema desempenhará um papel importantíssimo no desenvolvimento do texto e para completude apresentaremos sua demonstração

Lema 1.5.7 ([25] Teorema 17) Seja $\left(A_{\alpha} \mid \alpha \leq \mu\right)$ uma familia de módulos, e seja $\left(f_{\alpha \beta} \mid \alpha \leq \beta \leq \mu\right)$ uma seqüência de monomorfismo tais que $\left\{\left(A_{\alpha}, f_{\alpha \beta}\right) \mid \alpha \leq \beta \leq \mu\right\}$ forma um sistema direto contínuo.

Seja $C \in \operatorname{Mod} R$ tal que

$\operatorname{Ext}^{1}\left(A_{\alpha+1} / f_{\alpha \alpha+1}\left(A_{\alpha}\right), C\right)=0$ para todo $\alpha+1 \leq \mu$. Então $\operatorname{Ext}^{1}\left(A_{\mu}, C\right)=0$.

Prova: A demonstração será feita por indução transfinita. Provaremos que para um ordinal $\beta \leq \mu, \operatorname{Ext}^{1}\left(A_{\beta}, C\right)=0$.

Como por hipótese $\operatorname{Ext}^{1}\left(A_{0}, C\right)=0$ basta verificar os passos indutivos.

1) Se $\beta$ é sucessor de algum ordinal $\gamma$, isto é, $\beta=\gamma+1$. Supomos que $\operatorname{Ext}^{1}\left(A_{\gamma}, C\right)=$ 0 e consideremos a seqüência exata

$$
0 \longrightarrow A_{\gamma} \stackrel{f_{\gamma \beta}}{\longrightarrow} A_{\beta} \longrightarrow \frac{A_{\beta}}{f_{\gamma \beta}\left(A_{\gamma}\right)} \longrightarrow 0
$$

Da seqüência longa do cohomologia temos que $\operatorname{Ext}^{1}\left(A_{\beta}, C\right)=0$

2) Se $\beta$ é um ordinal limite. Mostraremos novamente por uma indução transfinita que a seqüência 1.2 cinde.

$$
0 \longrightarrow A \stackrel{\iota}{\longrightarrow} N \stackrel{\pi}{\longrightarrow} A_{\beta} \longrightarrow 0
$$

Para verificar que 1.2 cinde definiremos por indução transfinita uma seqüência contínua crescente de homomorfismos $\rho_{\alpha}: A_{\alpha} \longrightarrow N$ tais que $\pi \circ \rho_{\alpha}=f_{\alpha \beta}$. Suponha que $\rho_{\alpha}$ esteja definido para $\alpha<\tau$. Se $\tau$ é um ordinal limite então $\rho_{\tau}=\lim _{\alpha<\tau} \rho_{\alpha}$. Se $\tau=\gamma+1<\beta$ então por hipótese de indução existe $\sigma: A_{\tau} \longrightarrow N$ tal que $\pi \circ \sigma=f_{\tau \beta}$ 


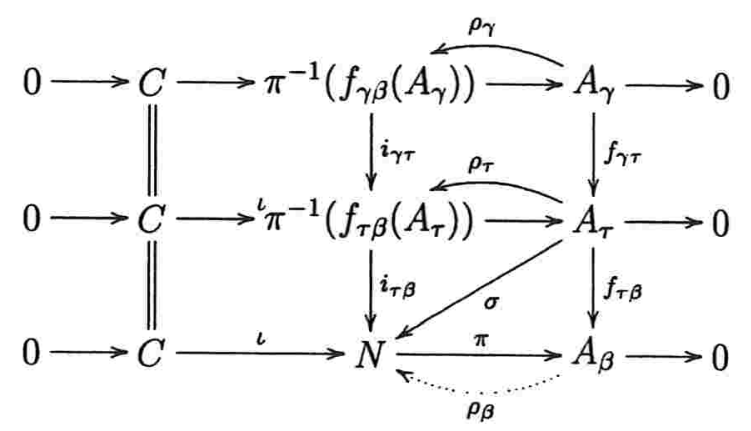

Temos também que $\rho_{\gamma}$ é uma cisão da primeira seqüência no Diagrama $1.3 \mathrm{e}$ os morfismos do tipo $i_{\gamma \beta}$ são inclusões de $\pi^{-1}\left(f_{\gamma \beta}\left(A_{\gamma}\right)\right)$ em $N$. Então $f^{-1} \pi i_{\gamma \beta} \rho_{\alpha}=$ $f^{-1} \pi i_{\gamma \beta} \rho_{\gamma}=i d_{\gamma}$ o que implica que $\pi i_{\gamma \beta} \rho_{\alpha}=f_{\gamma \beta}$. Além disso, $\sigma f_{\gamma \tau}: A_{\gamma} \longrightarrow N$ é tal que $\pi\left[i_{\gamma \beta} \rho_{\gamma}-\sigma f_{\gamma \tau}\right]=f_{\gamma \beta}-f_{\tau \beta} f_{\gamma \tau}=f_{\gamma \beta}-f_{\gamma \beta}=0$. Logo existe $\theta: A_{\gamma} \longrightarrow C$ tal que $i \theta=i_{\gamma \beta} \rho_{\gamma}-\sigma f_{\gamma \tau}$. Aplicando $\operatorname{Hom}(-, C)$ na seqüência

$$
0 \longrightarrow A_{\gamma} \longrightarrow A_{\tau} \longrightarrow \frac{A_{\tau}}{f_{\gamma \beta}\left(A_{\gamma}\right)} \longrightarrow 0
$$

obtemos

$$
\cdots \longrightarrow \operatorname{Hom}\left(A_{\tau}, C\right) \stackrel{\left(f_{\gamma \tau}\right)^{*}}{\longrightarrow} \operatorname{Hom}\left(A_{\gamma}, C\right) \longrightarrow \operatorname{Ext}^{1}\left(\frac{A_{\tau}}{f_{\gamma \beta}\left(A_{\gamma}\right)}, C\right)=0
$$

Portanto $\left(f_{\gamma \tau}\right)^{*}$ é sobrejetora e como conseqüência, $\theta$ se estende por $f_{\gamma \tau}$ a $\theta^{\prime}$, ou seja $\theta=\theta^{\prime} f_{\gamma \tau}$.

Definiremos $\rho_{\tau}$ pela seguinte expressão:

$$
\rho_{\tau}=\sigma+\iota \theta^{\prime}
$$

Observe que $\pi i_{\tau \beta}\left(\rho_{\tau} f_{\gamma \tau}\right)=f_{\tau \beta} f_{\gamma \tau}=f_{\gamma \beta}, \pi i_{\tau \beta} \rho_{\tau}\left(A_{\tau}\right) \subseteq f_{\tau \beta}\left(A_{\tau}\right)$ e $\pi i_{\tau \beta} \rho_{\tau} f_{\gamma \tau}\left(A_{\gamma}\right) \subseteq$ $f_{\alpha \beta}\left(A_{\gamma}\right)$ e que

$$
\rho_{\tau} f_{\gamma \tau}=\left(\sigma+\iota \theta^{\prime}\right) f_{\gamma \tau}=\sigma f_{\gamma \tau}+\iota \theta=\sigma f_{\gamma \tau}+\left[i_{\gamma \beta} \rho_{\gamma}-\sigma f_{\gamma \tau}\right]=i_{\gamma \beta} \rho_{\gamma},
$$

ou seja os quadros superiores do diagrama 


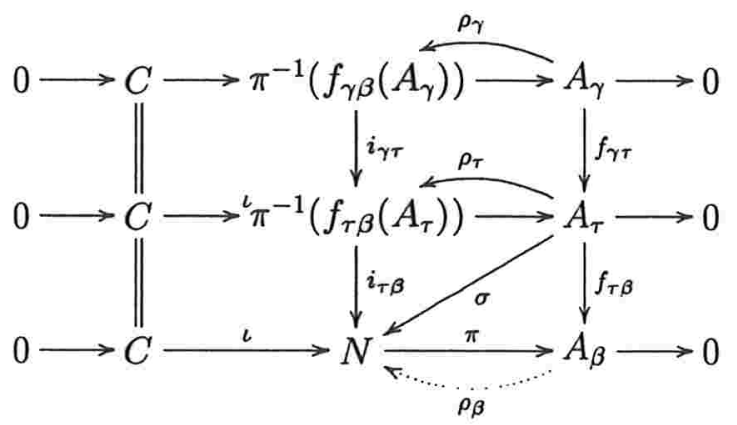

comutam. Se definirmos $\rho_{\beta}=\varliminf_{\overline{\alpha<\beta}}$, então $\pi \rho_{\beta}$ e $i d_{A_{\beta}}$ fazem comutar o diagrama de limite direto formado pelas linhas pontilhadas abaixo, então $\pi \rho_{\beta}=i d_{A_{\beta}}$, e disso segue que $\operatorname{Ext}^{1}\left(A_{\beta}, C\right)=0$

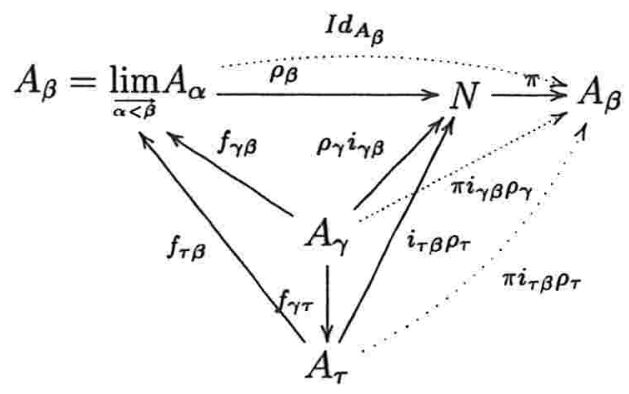

O próximo lema é bem conhecido em álgebra homológica e utiliza-se do argumento dimension shifting (veja [36]), entretanto apresentaremos aqui sua demostração por se tratar de um resultado essencial no desenvolvimento do texto.

\section{Lema 1.5.8 (Lema de Dimension Shifting)}

1) Sejam $A$ e $Y$ dois $R$-módulos. Se

$$
0 \longrightarrow A \stackrel{f_{0}}{\longrightarrow} B_{0} \stackrel{f_{1}}{\longrightarrow} B_{1} \stackrel{f_{2}}{\longrightarrow} B_{2} \stackrel{f_{3}}{\longrightarrow} \cdots \stackrel{f_{n}}{\longrightarrow} B_{n} \longrightarrow 0
$$

é uma seqüência exata com $B_{i} \in Y^{\perp}$ para $0 \leq i<n$, então

$$
\operatorname{Ext}^{i}\left(Y, B_{n}\right) \cong \operatorname{Ext}^{i+n}(Y, A)
$$

para cada $i>0$. Em particular, se $\operatorname{pd} Y \leq n$, então $B_{n} \in Y^{\perp}$. 
2) Sejam A e $Y$ dois $R$-módulos. Se

$$
0 \longrightarrow B_{n} \stackrel{g_{n}}{\longrightarrow} B_{n-1} \stackrel{g_{n-1}}{\longrightarrow} \cdots \stackrel{f_{2}}{\longrightarrow} B_{1} \stackrel{g_{1}}{\longrightarrow} B_{0} \stackrel{g_{0}}{\longrightarrow} A \longrightarrow 0
$$

é uma seqüência exata com $B_{i} \in^{\perp} Y$ para $0 \leq i<n$, então

$$
\operatorname{Ext}^{i}\left(B_{n}, Y\right) \cong \operatorname{Ext}^{i+n}(A, Y)
$$

para cada $i>0$. Em particular, se id $Y \leq n$, então $B_{n} \in^{\perp} Y$.

Prova: Faremos somente a prova de 1) sendo que 2) é dual.

Considere a seqüência $0 \longrightarrow A \longrightarrow B_{0} \longrightarrow K_{1} \longrightarrow 0$, onde $K_{1}=\operatorname{Im}\left(f_{1}\right)$. Aplicando o funtor $\operatorname{Hom}(Y,-)$ temos a seqüência exata

$$
\cdots \longrightarrow \operatorname{Ext}^{i}(Y, A) \longrightarrow \operatorname{Ext}^{i}\left(Y, B_{0}\right) \longrightarrow \operatorname{Ext}^{i}\left(Y, K_{1}\right) \longrightarrow \operatorname{Ext}^{i+1}(Y, A) \longrightarrow 0
$$

Como $\operatorname{Ext}^{i}\left(Y, B_{0}\right)=\operatorname{Ext}^{i+1}\left(Y, B_{0}\right)=0$, então $\operatorname{Ext}^{i}\left(Y, K_{1}\right) \cong \operatorname{Ext}^{i+1}(Y, A)$. Seguindo por indução temos para $K_{j}=\operatorname{Im}\left(f_{i j}\right)$, a sequiência

$$
0 \longrightarrow K_{j} \longrightarrow B_{j} \longrightarrow K_{j+1} \longrightarrow 0 \text {. }
$$

Aplicando novamente o funtor $\operatorname{Hom}\left(Y_{-}\right)$temos pela seqüência exata longa de homologia que $\operatorname{Ext}^{i}\left(Y, K_{j+1}\right) \cong \operatorname{Ext}^{i+1}\left(Y, K_{j}\right)$. Observe que $K_{n}=B_{n}$, logo $\operatorname{Ext}^{i}\left(Y, B_{n}\right) \cong$ $\operatorname{Ext}^{i+n}(Y, A)$.

Finalmente, se $\operatorname{pd} Y \leq n$, então $\operatorname{Ext}^{i+n}(Y, A)=0$ e temos que $B_{n} \in Y^{\perp}$.

O próximo corolário é decorrência do Lema 1.5 .7 e do Lema de Dimension Shifting 1.5.8 acima, entretanto é uma pequena contribuição desse trabalho e é de essencial importância para o desenvolvimento do texto, pois estende o Lema 1.5.7 para todos os funtores Ext ${ }^{i}$.

Corolário 1.5.9 Seja $\left(A_{\alpha} \mid \alpha \leq \mu\right)$ uma seqüência de módulos, e seja $\left(f_{\alpha \beta} \mid \alpha \leq \beta \leq\right.$ $\mu$ ) uma seqüência de monomorfismo tais que $\left\{\left(A_{\alpha}, f_{\alpha \beta}\right) \mid \alpha \leq \beta \leq \mu\right\}$ é um sistema direto contínuo.

Seja $C \in \operatorname{Mod} R$ tal que tal que $\operatorname{Ext}^{i}\left(A_{\alpha+1} / f_{\alpha \alpha+1}\left(A_{\alpha}\right), C\right)=0$ para todo $\alpha+1 \leq \mu$. Então $\operatorname{Ext}^{i}\left(A_{\mu}, C\right)=0$. 
Prova: Consideremos a seqüência exata obtida de uma corresolução injetiva de $C$

$$
0 \longrightarrow C \longrightarrow I_{0} \longrightarrow I_{1} \longrightarrow I_{2} \longrightarrow \cdots \longrightarrow I_{i-1} \longrightarrow Q_{i-1} \longrightarrow 0 \text {. }
$$

Então pelo Lema de Dimension Shifting

$$
0=\operatorname{Ext}^{i}\left(A_{\alpha+1} / f_{\alpha \alpha+1}\left(A_{\alpha}\right), C\right) \cong \operatorname{Ext}^{1}\left(A_{\alpha+1} / f_{\alpha \alpha+1}\left(A_{\alpha}\right), Q_{i-1}\right) .
$$

Pelo Lema 1.5.7, $\operatorname{Ext}^{1}\left(A_{\mu}, Q_{i-1}\right)=0$. Usando novamente o Lema de Dimension Shifting temos $\operatorname{Ext}^{i}\left(A_{\mu}, C\right)=0$.

Lema 1.5.10 Seja $\left\{X_{i}, \phi_{j}^{i}\right\}_{i \in I}$ um sistema direto de módulos, onde $(I, \leq)$ é um conjunto dirigido parcialmente ordenado e toda cadeia em I é infinita. Suponha que para cada cadeia $\mathcal{C} \subset I$, exista apenas um número finito de indices $i \in \mathcal{C}$ tais que $X_{i} \neq 0$. Então $\lim _{\bar{i} \in \mathbb{I}}\left(X_{i}\right) \cong 0$. Em particular se $I=\mathbb{N}, I$ é totalmente ordenado e possui uma única cadeia $\mathcal{C}=\mathbb{N}$. Segue que se $X_{i}=0$ para todo $i \geq n_{0}$, então $\lim _{\bar{i} \in \mathbb{N}} X_{i}=0$.

Prova: Vamos mostrar diretamente que $\lim _{\bar{i} \in \mathbb{1}}\left(X_{i}\right)=0$. Pela definição de sistema direto, se $i \leq k \leq j$, então o diagrama

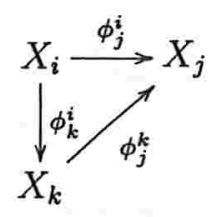

comuta. Como toda cadeia $\mathcal{C}$ em $I$ deve ser infinita, existe um índice $j_{0} \in \mathcal{C}$ tal que $X_{j} \cong 0$ para todo $j \geq j_{0}$.

O limite de um sistema direto de módulos é um $R$-módulo, denotado por $\lim _{i \in I}\left(X_{i}\right)$, e um conjunto de homomorfismos $\alpha_{i}: X_{i} \rightarrow \lim _{i \in I}\left(X_{i}\right)$ comutando o diagrama

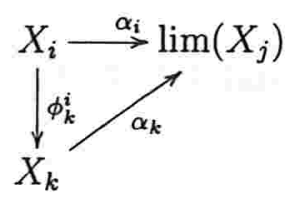

satisfazendo a propriedade universal: 
Para qualquer $X$ e homomorfismos $\beta_{i}: X_{i} \rightarrow X$ comutando o diagrama

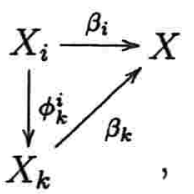

existe um único $\alpha: \lim _{i \in l}\left(X_{j}\right) \rightarrow X$ tal que o diagrama abaixo comuta.

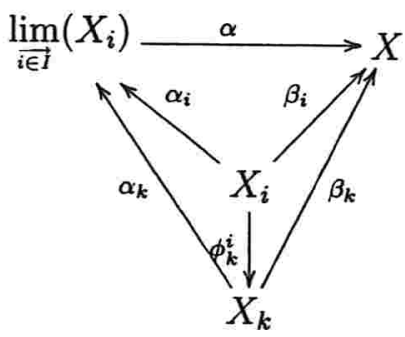

Dado $X$ como acima, como toda cadeia $\mathcal{C} \subseteq I$ é infinita e só possui um número finito de índices $i \operatorname{com} X_{i} \neq 0$, então $\beta_{i}$ é nulo, pois $\beta_{i}$ fatora-se através de algum $X_{j} \cong 0$ para algum $j>i$ em $\mathcal{C}$. Segue que $\beta_{i}=0$ para qualquer $i \in I$.

Se considerarmos $X=\lim _{\bar{i} \in \mathbb{I}}\left(X_{i}\right)$ no diagrama (1.5), temos que cada $\alpha_{i}$ fatora-se através de algum $\phi_{j}^{i} \operatorname{com} X_{j} \cong 0$, então $\alpha_{i}=0$ para todo $i \in I$. Logo o morfismo nulo

$$
\alpha=0: \lim _{\bar{i} \in I}\left(X_{i}\right) \rightarrow \lim _{\bar{i} \in I}\left(X_{i}\right)
$$

faz o diagrama (1.5) comutar. Como o morfismo identidade

$$
\text { id }: \lim _{i \in I}\left(X_{i}\right) \rightarrow \lim _{i \in I}\left(X_{i}\right)
$$

também faz o diagrama (1.5) comutar, segue pela propriedade universal que $0=$ $\alpha=$ id. Mas id $=0$ se e somente se $\underset{i \in I}{\lim }\left(X_{i}\right)=0$.

\subsection{Teoria de Auslander Reiten}

Apresentaremos agora um resumo da teoria de Auslander Reiten que utilizaremos no decorrer do trabalho, ressaltamos porém, que para uma melhor compreensão do 
assunto, que uma bibliografia mais especializada seja consultada, sugerimos [13] ou [4] para uma introdução mais detalhada dessa teoria.

Vimos no início do capítulo que $\mathbb{K}$-álgebras (básicas e de dimensão finita) podem ser representadas por quivers, ou de outra forma como sendo isomorfas a quociente de $\mathbb{K}$-álgebras de caminhos. Veremos agora como podemos representar a categoria ind $A$ para uma $\mathbb{K}$-álgebra $A$.

Apresentaremos abaixo um rápida noção da teoria e Auslander Reiten para um $\mathbb{K}$-álgebra, entretanto, lembramos que essa teoria é válida para álgebras de Artin. Mas, como a utilizaremos somente no contexto de $\mathbb{K}$-álgebras, não apresentaremos no contexto de álgebras de Artin, pois para $\mathbb{K}$-álgebras a descrição dos morfismos irredutíveis é facilitada e simplifica a descrição do quiver de Auslander Reiten. No caso das álgebras de Artin é necessário trabalharmos com quivers valuados (Veja [13]).

Seja $A$ uma $\mathbb{K}$-álgebra e $\bmod A$ a categoria dos módulos finitamente gerados sobre $A$. Uma seção (retração) em $\bmod A$ é um morfismo que admite um inverso à esquerda (direita) em $\bmod A$.

Definição 1.6.1 Seja A uma $\mathbb{K}$-álgebra

(i) Um morfismo $f: L \longrightarrow M$ é dito ser minimal à esquerda se para todo $h \in \operatorname{Hom}(M, M)$ tal que $h f=f$ é um automorfismo.

(ii) Um morfismo $g: M \longrightarrow N$ é dito ser minimal à direita se para todo $k \in$ $\operatorname{Hom}(M, M)$ tal que $g k=g$ é um automorfismo.

(iii) Um morfismo $f: L \longrightarrow M$ é chamado de quase cindido à esquerda se:

a) $f$ não é uma seção.

b) Para todo $u: L \longrightarrow V$ que não seja uma seção, existe $u^{\prime}: M \longrightarrow U$ tal que $u^{\prime} f=u$

(iv) Um morfismo $g: M \longrightarrow N$ é chamado de quase cindido à direita se:

a) $f$ não é uma retração.

b) Para todo $v: V \longrightarrow N$ que não seja uma retração, existe $v^{\prime}: V \longrightarrow M$ tal que $g v^{\prime}=v$ 
(v) Um morfismo $f: L \longrightarrow M$ é chamado de minimal quase cindido à esquerda se for ambos: minimal e quase cindido à esquerda.

(vi) Um morfismo g: $M \longrightarrow N$ é chamado de minimal quase cindido à direita se for ambos: minimal e quase cindido à direita.

Definição 1.6.2 Um morfismo $f: X \longrightarrow Y$ em $\bmod A$ é irredutível se

a) $f$ não é nem seção nem retração.

b) Se $f=f_{1} f_{2}$ então $f_{1}$ é uma retração ou $f_{2}$ é uma seção.

Sejam $A$ uma $\mathbb{K}$-álgebra e $X, Y A$-módulos finitamente gerados. Definimos $\operatorname{rad}(X, Y)$, o radical de $\operatorname{Hom}(X, Y)$ por $\operatorname{rad}(X, Y)=\{f \in \operatorname{Hom}(X, Y) / h f g$ não é um isomorfismo para quaisquer $g: M \rightarrow X$ e $h: Y \rightarrow M \operatorname{com} M \in \operatorname{ind} A\}$.

Para quaisquer $X$ e $Y$ em $\bmod A$ e $n \in \mathbb{N}$, definimos a $n$-ésima potência do radical indutivamente por $\operatorname{rad}(X, Y)^{n}=\{f \in \operatorname{Hom}(X, Y)$ tais que existem $M \in$ $\bmod A$ e morfismos $g \in \operatorname{rad}(X, M)$ e $h \in \operatorname{rad}^{n-1}(M, Y)$ com $\left.f=h g\right\}$ e finalmente $\operatorname{rad}^{\infty}(X, Y)=\bigcap_{n \in \mathbb{N}} \operatorname{rad}^{n}(X, Y)$.

Proposição 1.6.3 Sejam $X$ e $Y$ módulos irredutíveis em $\bmod A$. Um morfismo $f: X \longrightarrow Y$ é irredutível se e somente se $f \in \frac{\operatorname{rad}(X, Y)}{\operatorname{rad}^{2}(X, Y)}$

Definição 1.6.4 Um seqüência exata curta em $\bmod A$

$$
0 \longrightarrow L \stackrel{f}{\longrightarrow} M \stackrel{g}{\longrightarrow} N \longrightarrow 0
$$

é chamada de seqüência quase cindida ou seqüência de Auslander Reiten se:

a) $f$ é um morfismo minimal quase cindido à esquerda.

b) g é um morfismo minimal quase cindido à direita. 
As seqüências quase cindidas estão relacionadas aos funtores de translação de Auslander Reiten $\tau=D \operatorname{Tr}$ e $\tau^{-}=\operatorname{Tr} D$. Essa relação se dá seguinte forma. Se

$$
0 \longrightarrow L \stackrel{f}{\longrightarrow} M \stackrel{g}{\longrightarrow} N \longrightarrow 0
$$

for uma seqüência quase cindida, então $L$ e $N$ são indecomponíveis e $L \cong \tau N$ e $N \cong \tau^{-} L$. Veja [13] ou [4] para maiores detalhes.

As translações de Auslander Reiten $\tau: \underline{\bmod } A \rightarrow \overline{\bmod } A$ e $\tau^{-}: \overline{\bmod } A \rightarrow \underline{\bmod } A$ são equivalências quase inversas entre essas categorias. Pode se concluir a partir disso que todo módulo não projetivo $M$ tem um transladado $\tau M$ e todo módulo não injetivo $N$ tem um transladado $\tau^{-} N$.

Essas propriedades permitem construir um quiver $\Gamma$ para representar a categoria $\operatorname{ind} A$ dos módulos indecomponíveis de $\bmod A$. Esse quiver é conhecido como quiver da Auslander Reiten e é definido da seguinte forma: Os vértice de $\Gamma$ correspondem bijetivamente às classe de isomorfismos $[M]$ dos módulo indecomponíveis $M$ de $\bmod A$. As flechas entre $[M]$ e $[N]$ estão em correspondência bijetiva com os vetores uma base do $\mathbb{K}$-espaço vetorial $\frac{\operatorname{rad}(M, N)}{\operatorname{rad}(M, N)^{2}}$. Uma componente conexa $\mathbb{P}$ do quiver de Auslander Reiten $\Gamma$ é chamada de pós-projetiva se for acíclica e todo vértice $[M] \in \mathbb{P}$ for da forma $\left[\tau^{n} P\right]$ para algum projetivo indecomponível $P$ e algum $n \in \mathbb{N}$. Analogamente, uma componente conexa II do quiver de Auslander Reiten é chamada de pré-injetiva se for acíclica e todo vértice $[M] \in \mathbb{I}$ for da forma $\left[\tau^{-n} I\right]$ para algum projetivo indecomponível $I$ e algum $n \in \mathbb{N}$.

Para finalizar esse capítulo seguem abaixo alguns resultados que serão utilizados nos capítulos seguintes. As demonstrações podem ser encontradas em [13] ou [1].

Lema 1.6.5 Sejam A uma $\mathbb{K}$-Álgebra e $M$ um A-módulo, então:

i) $\operatorname{pd} M \leq 1$ se e somente se $\operatorname{Hom}(D A, \tau M)=0$.

ii) $\operatorname{id} M \leq 1$ se e somente se $\operatorname{Hom}\left(\tau^{-} M, A\right)=0$.

Proposição 1.6.6 (Fórmula de Auslander Reiten) Sejam A uma KK-álgebra e $M, N$ dois $A$-módulos, então existe um isomorfismo

$$
\operatorname{Ext}^{1}(M, N) \cong D \underline{\operatorname{Hom}}\left(\tau^{-} N, M\right) \cong D \overline{\operatorname{Hom}}(N, \tau M)
$$

funtorial em ambas as variáveis. 
Corolário 1.6.7 Nas hipóteses do teorema acima valem as seguintes afirmações:

i) $S e \operatorname{pd} M \leq 1$ e $N$ é um módulo arbitrário, então existe um isomorfismo

$$
\operatorname{Ext}^{1}(M, N) \cong D \operatorname{Hom}(N, \tau M) \text {. }
$$

ii) $S e \operatorname{id} N \leq 1$ e $M$ é módulo arbitrário, então $\operatorname{Ext}^{1}(M, N) \cong D \operatorname{Hom}\left(\tau^{-} N, M\right)$. 


\section{Capítulo 2}

\section{Teoria Tilting Infinitamente Gerada}

Este capítulo foi desenvolvido para recordar a teoria tilting para módulos infinitamente gerados e apresentar novos resultados que generalizam antigos resultados que, até então, só estavam provados para módulos fintamente gerados.

A teoria tilting infinitamente gerada é uma generalização natural da teoria tilting clássica [17] e [29], e da teoria tilting de dimensão projetiva finita [32], para o contexto da categoria $\operatorname{Mod} R$. A teoria tilting infinitamente gerada foi introduzida inicialmente por Colpi e Trilifaj em [22] para dimensão projetiva menor ou igual a 1, estendendo a noção clássica para $\operatorname{Mod} R$ e estendida, posteriormente, por AngeleriHügel e Coelho para dimensão projetiva finita [6].

A maioria dos conceitos que generalizamos aqui puderam ser obtidos sem grandes problemas a partir dos seus análogos ou duais finitamente gerados, mas para isso contamos com uma ajuda bastante importante, o teorema de existência de préenvolvente, que garante que qualquer classe do tipo $M^{\perp}$ é pré-envolvente (veja a proposição 1.4.3). Esse resultado foi demonstrado por Eklof e Trlifaj em [25] Teorema 10 e vem permitindo um avanço significativo na teoria tilting infinitamente gerada. Outro fator decisivo foi o teorema de existência de pré-coberturas para qualquer classe do tipo ${ }^{\perp} C$ com $C$ um módulo cotilting 1.4.4, resultado esse que é uma decorrência de [2] 2.1,2.2,2.3 e 2.5 e foi demonstrado por Angeleri-Hügel e Coelho em [6] Proposição 3.3. Vale lembrar que ainda é um problema em aberto quando uma classe do tipo ${ }^{\perp} M$ é uma classe de recobrimento para um módulo $M$ qualquer. Outro ponto importante desse capítulo é a existência de complemento de módulos tilting parciais de tipo finito e a obtenção de alguns módulos tiltings maximais. 
Ao longo do capítulo $R$ denotará um anel com unidade e quando nada for mencionado os módulos serão módulos em $\operatorname{Mod} R$.

\subsection{Definições e resultados iniciais}

Nessa seção apresentaremos a definição de módulos tiltings e cotilting e descreveremos algumas propriedades sobre essas classe de módulos.

Dizemos que um $R$-módulo $M$ é sincero se todos os $R$-módulos simples aparecem como fator de composição de $M$ e $M$ é dito ser fiel se ann $M=0$, em particular se existe um monomorfismo $f: A \longrightarrow M^{(I)}$ para algum conjunto $I$, então $M$ é fiel. Algumas das proposições e lemas serão apresentados com demonstrações devido a sua grande importância no texto ou porque essas demonstrações possuem idéias e argumentos muito utilizados do desenvolvimento do texto.

Definição 2.1.1 Seja $n<\infty$. Um módulo $T$ é $n$-tilting se satisfaz:

$\left.\mathbf{T}_{1}\right) \operatorname{pd} T \leq n$

$\left.\mathrm{T}_{2}\right) \operatorname{Ext}_{R}^{i}\left(T, T^{(I)}\right)=0$ para cada $i \geq 1$ e para todo conjunto de índices I;

$\mathbf{T}_{3}$ ) Existe $r>0$ e uma seqüência exata longa

$$
0 \longrightarrow R \stackrel{f_{0}}{\longrightarrow} T_{0} \stackrel{f_{1}}{\longrightarrow} \cdots \stackrel{f_{r}}{\longrightarrow} T_{r} \longrightarrow 0
$$

com $T_{i} \in \operatorname{Add} T$ para cada $0 \leq i \leq r ;$

$O$ módulo $T$ é chamado de tilting parcial se satisfaz somente os ítens $\mathbf{T}_{1}$ ) e $\mathbf{T}_{2}$ ). Dizemos que uma classe de módulos $\mathcal{T}$ é $n$-tilting se existe um módulo $n$-tilting $T$ tal que $\mathcal{T}=T^{\perp}$ e que $T$ é de tipo finito se $T^{\perp}$ é uma classe de tipo finito.

Observe que todo módulo tilting é fiel e sincero por $\mathrm{T}_{3}$.

Como todo par de cotorção cogerado por um conjunto é completo, então o par de cotorção $\mathcal{C}=\left({ }^{\perp} \mathcal{T}, \mathcal{T}\right)$ é completo pela Proposição 1.4.3, visto que $\mathcal{C}$ é gerada pelo conjunto de syzygies de uma resolução projetiva de $T$.

Definição 2.1.2 Seja $n<\infty$. Um módulo $T$ é $n$-cotilting se satisfaz: 
$\left.\mathrm{C}_{1}\right) \operatorname{id} T \leq n$

$\left.\mathrm{C}_{2}\right) \operatorname{Ext}_{R}^{i}\left(T^{I}, T\right)=0$ para todo $i \geq 1$ e para todo conjunto de indices $I$;

$\mathrm{C}_{3}$ ) Existe $r>0$ e uma seqüência exata longa

$$
0 \longrightarrow T_{r} \stackrel{f_{r}}{\longrightarrow} \cdots \longrightarrow T_{1} \stackrel{f_{1}}{\longrightarrow} T_{0} \stackrel{f_{0}}{\longrightarrow} Q \longrightarrow 0
$$

com $T_{i} \in \operatorname{Prod} T$ para cada $0 \leq i \leq r$ e $Q$ um cogerador injetivo de $\operatorname{Mod} R$;

Dizemos que uma classe de módulos $\mathcal{T}$ é $n$-cotilting se existe um módulo $n$ cotilting $T$ tal que $\mathcal{T}={ }^{\perp} T$.

Lema 2.1.3 ([6] Lema 2.2) Seja $M \in \operatorname{Mod} R$.

a) Denote por $\mathcal{X}=M^{\perp}$. Se $\operatorname{pd} M=n$, então para todo $Y \in{ }^{\perp} \mathcal{X} \operatorname{pd} Y \leq n$;

b) Denote por $\mathcal{X}={ }^{\perp} M . S e$ id $M=n$, então para todo $Y \in \mathcal{X}^{\perp}$ id $Y \leq n$;

Prova: a) Seja $A \in \operatorname{Mod} R$ e considere uma seqüência exata longa

$$
0 \longrightarrow A \longrightarrow I_{0} \longrightarrow \cdots \longrightarrow I_{n-1} \longrightarrow Q_{n} \longrightarrow 0
$$

com $I_{j}$ módulos injetivos, portanto pertencentes a $\mathcal{X}$. Como pd $M \leq n$, pelo Lema 1.5.8, $Q_{n} \in \mathcal{X}$. Se $Y \in \in^{\perp} \mathcal{X}$, então $I_{j}, Q_{n} \in Y^{\perp}$. Segue novamente pelo Lema 1.5.8 que $\operatorname{Ext}^{i+n}(Y, A)=0$ para todo $i>0$. Portanto $\operatorname{pd} Y \leq n$. A prova de (b) é dual. 
Observações:

1. Se $T$ é um módulo tilting, então $T^{\perp} \subseteq$ GenT. Veja [6] Lema 2.3) a);

2. Se $T$ é um módulo cotilting, então ${ }^{\perp} T \subseteq \operatorname{Cogen} T$.Veja [6] Lema 2.3) b);

Lema 2.1.4 ([6] Lema 2.4)) Seja $M$ um R-módulo.

(a) Suponha que $M$ satisfaça a condição $\mathrm{T}_{2}$ ) da definição de módulo tilting e denote $M^{\perp}$ por $\mathcal{X}$. Se $\mathcal{X} \subset$ GenM, então:

(i) Para cada $X \in \mathcal{X}$, existe uma seqüência exata curta,

$$
0 \longrightarrow K \longrightarrow M^{\prime} \longrightarrow X \longrightarrow 0
$$

com $M^{\prime} \in \operatorname{Add} M$ e $K \in \mathcal{X}$;

(ii) Todo morfismo $A \rightarrow X$ com $A \in^{\perp} \mathcal{X} e X \in \mathcal{X}$ fatora-se através de Add $M$. Em particular $\operatorname{Add} M=\mathcal{X} \cap^{\perp} \mathcal{X}$;

(b) Suponha $M$ satisfaça a condição $C_{2}$ ) da definição de módulo cotilting e denote ${ }^{\perp_{M}}$ por $\mathcal{X}$. Se $\mathcal{X} \subseteq \operatorname{Cogen} M$, então:

(i) Para cada $X \in \mathcal{X}$, existe uma seqüência exata curta

$$
0 \longrightarrow X \longrightarrow M^{\prime} \longrightarrow L \longrightarrow 0
$$

$\operatorname{com} M^{\prime} \in \operatorname{Prod} M$ e $L \in \mathcal{X}$;

(ii) Todo morfismo $X \rightarrow C$ com $C \in \mathcal{X}^{\perp}$ e $X \in \mathcal{X}$ fatora-se através de $\operatorname{Prod} M$. Em particular, temos que $\operatorname{Prod} M=\mathcal{X} \cap \mathcal{X}^{\perp}$;

Prova: (a) (i) Seja $X \in \mathcal{X}$ e considere uma Add $M$-pré-cobertura $\left(M^{\prime}, g\right)$ de $X$. Como $X \in \operatorname{Gen} M$, então $g$ é um epimorfismo. Vejamos que $K=\operatorname{Ker}(g) \in \mathcal{X}$. De fato, observemos que $\operatorname{Ext}^{1}(M, K)=0$, pois $\operatorname{Hom}(M, g)$ é um epimorfismo e $\operatorname{Ext}^{i+1}\left(M, M^{\prime}\right)=0$ por hipótese. Para $i \geq 1$ considere a seqüência exata longa de homologia

$$
\cdots \longrightarrow \operatorname{Ext}^{i}(M, X) \longrightarrow \operatorname{Ext}^{i+1}(M, K) \longrightarrow \operatorname{Ext}^{i+1}\left(M, M^{\prime}\right) \longrightarrow \cdots
$$


sendo $X \in \mathcal{X}, \operatorname{Ext}^{i}(M, X)=0$ e de $\left.\mathrm{T}_{2}\right)$ segue que $\operatorname{Ext}^{i+1}\left(M, M^{\prime}\right)=0$. Portanto $\operatorname{Ext}^{i}(M, K)=0$. Para todo $i \geq 1$.

(ii) Seja agora $f: A \rightarrow X \operatorname{com} A \in^{\perp} \mathcal{X}$ e $X \in \mathcal{X}$ e considere a seqüência exata curta

$$
0 \longrightarrow K \longrightarrow M^{\prime} \stackrel{g}{\longrightarrow} X \longrightarrow 0
$$

com $M^{\prime} \in \operatorname{Add} M$. Como $\operatorname{Ext}^{1}(A, K)=0, f$ fatora-se através de $g$ como queríamos. Para mostrar que Add $M=\mathcal{X} \cap \mathcal{X}^{\perp}$, observe que $A \in \mathcal{X} \cap \mathcal{X}^{\perp}$, então a aplicação identidade de $A$ fatora-se através de $\operatorname{Add} M$ e, assim, $A \in \operatorname{Add} M$. A outra inclusão segue por $\mathrm{T}_{2}$ ). A prova de (b) é dual.

Classes tilting podem ser caracterizadas pelo seguinte resultado.

Teorema 2.1.5 ([6] 4.2 e 4.3)

1) Uma classe de módulos $\mathcal{T}$ é $n$-tilting se e somente se:

a) $\left({ }^{\perp} \mathcal{T}, \mathcal{T}\right)$ é um par de cotorção completo;

b) $\mathcal{T}$ é fechada para somas diretas arbitrárias, e $\mathcal{T}$ é fechada para conúcleos de monomorfismos;

c) ${ }^{\perp} \mathcal{T} \subseteq \mathcal{P}_{n}$

2) Uma classe de módulos $\mathcal{F}$ é n-cotilting se e somente se:

a) $\left(\mathcal{F}, \mathcal{F}^{\perp}\right)$ é um par de cotorção completo;

b) $\mathcal{F}$ é fechada para produtos diretos, e para núcleos de epimorfismos;

c) $\mathcal{F}^{\perp} \subseteq \mathcal{I}_{n}$

Prova: Faremos a prova de 1) sendo que 2) é dual.

$\left(\Rightarrow\right.$ ) Suponha $\mathcal{T}$ uma classe $n$-tilting, então $\mathcal{T}=T^{\perp}$ para algum $R$-módulo tilting $T$.

a) $\mathrm{O} \operatorname{par}\left({ }^{\perp} \mathcal{T}, \mathcal{T}\right)$ é uma par de cotorção cogerado por um conjunto. Segue pela Proposição 1.4.3 que $\left({ }^{\perp} \mathcal{T}, \mathcal{T}\right)$ é completo. 
b) Seja $0 \longrightarrow X \longrightarrow Y \longrightarrow Z \longrightarrow 0$ com $X$ e $Y \in \mathcal{T}$, então $\operatorname{Ext}^{i}(T, X)=$ $0=\operatorname{Ext}^{i}(T, Y)$. Da seqüência exata longa de homologia

$$
\operatorname{Ext}^{i-1}(T, Z) \longrightarrow \operatorname{Ext}^{i}(T, X) \longrightarrow \operatorname{Ext}^{i}(T, Y) \longrightarrow \operatorname{Ext}^{i}(T, Z) \longrightarrow \operatorname{Ext}^{i+1}(T, X)
$$

temos que $\operatorname{Ext}^{i}(T, Z)=0$ para todo $i>0$. Portanto $\mathcal{T}$ é fechada para conúcleos de monomorfismos.

Considere agora uma família $\left\{X_{i}\right\}_{i \in I} \subseteq \mathcal{T}$. Como cada $X_{i} \in \mathcal{T}$, existe, para cada $i \in I$, uma seqüência exata

$$
0 \longrightarrow K_{i}^{1} \longrightarrow T_{i}^{1} \longrightarrow X_{i}^{1} \longrightarrow 0
$$

com $K_{i}^{1} \in \mathcal{T}$ e $T_{i}^{1} \in \operatorname{Add} T$, por 2.1.4. Então podemos construir uma seqüência exata

$$
0 \longrightarrow \coprod_{i \in I} K_{i}^{1} \longrightarrow \coprod_{i \in I} T_{i}^{1} \longrightarrow \coprod_{i \in I} X_{i} \longrightarrow 0
$$

como coproduto das seqüências em (2.1). Sendo que cada $K_{i}^{1} \in \mathcal{T}$, repetindo o argumento anterior podemos construir outra seqüência exata

$$
0 \longrightarrow \coprod_{i \in I} K_{i}^{2} \longrightarrow \coprod_{i \in I} T_{i}^{2} \longrightarrow \coprod_{i \in I} K_{i}^{1} \longrightarrow 0 \text {. }
$$

Seguindo por indução, conseguimos construir uma seqüência exata longa

$$
0 \longrightarrow \coprod_{i \in I} K_{i}^{n} \longrightarrow \coprod_{i \in I} T_{i}^{n} \longrightarrow \cdots \longrightarrow \coprod_{i \in I} T_{i}^{1} \longrightarrow \coprod_{i \in I} X_{i} \longrightarrow 0
$$

$\operatorname{com} \coprod_{i \in I} T_{i}^{m} \in \operatorname{Add} T$ para cada $1 \leq m \leq n$. Segue pelo Lema 1.5.8 que

$$
\operatorname{Ext}^{j+n}\left(T, \coprod_{i \in I} K_{i}^{n}\right) \cong \operatorname{Ext}^{j}\left(T, \coprod_{i \in I} X_{i}\right)
$$

Mas, pd $T \leq n$, portanto $\operatorname{Ext}^{j+n}\left(T, \coprod_{i \in I} K_{i}^{n}\right)=0$ e então $\operatorname{Ext}^{j}\left(T, \coprod_{i \in I} X_{i}\right)=0$. Finalmente, c) segue pelo Lema 2.1.3.

Teorema 2.1.6 Sejam $R$ um anel e $n$ um número inteiro positivo.

As classes $n$-tiltings de tipo finito de $\operatorname{Mod} R$ correspondem bijetivamente às subclasses resolventes de $\mathcal{P}_{n} \cap \mathrm{FP}_{\infty}(R)$. Essa associação é dada pelas aplicações inversas $\alpha: \mathcal{T} \rightarrow^{\perp} \mathcal{T} \cap \mathrm{FP}_{\infty}(R)$ e $\beta: \mathcal{C} \rightarrow \mathcal{C}^{\perp}$. 
Prova: Para qualquer classe $n$-tilting temos ${ }^{\perp} \mathcal{T} \subseteq \mathcal{P}_{n}$, pelo Lema 2.1.3. Dessa forma $\alpha(\mathcal{T})$ é uma subclasse resolvente de $\mathcal{P}_{n} \cap F_{\infty}(R)$. Portanto $\alpha$ está bem definida. Suponha agora que $\mathcal{C} \subseteq \mathcal{P}_{n}^{\infty} \cap \mathrm{FP}_{\infty}(R)$ uma subclasse, então $\mathcal{C}^{\perp}$ é fechada para coprodutos pelo Corolário 1.5.5 e $\left({ }^{\perp}\left(\mathcal{C}^{\perp}\right), \mathcal{C}^{\perp}\right)$ é um par de cotorção completo. Além disso $\mathcal{C}^{\perp}$ é fechada para conúcleos de monomorfismos e ${ }^{\perp}\left(\mathcal{C}^{\perp}\right) \subseteq \mathcal{P}_{n}$, então por [6] Teorema 4.1, $\mathcal{C}^{\perp}$ é uma classe $n$-tilting de tipo finito. Logo $\beta$ está bem definida.

Seja agora $\mathcal{T}$ uma classe $n$-tilting de tipo finito e $\mathcal{S} \subset F P_{\infty}(R)$ tal que $\mathcal{S}^{\perp}=\mathcal{T}$. Então $\mathcal{S} \subset \alpha(\mathcal{T})$. Por outro lado, $\alpha(\mathcal{T})={ }^{\perp} \mathcal{T} \subset\left({ }^{\perp} \mathcal{T}\right)^{\perp} \subset \alpha(\mathcal{T})^{\perp}=\beta \alpha(\mathcal{T})$, então $\mathcal{S} \subset \mathcal{P}_{n}$ e $\beta \alpha=i d$. Reciprocamente se $\mathcal{C}$ é resolvente e $\mathcal{C} \subset \mathcal{P}_{n} \cap \mathrm{FP}_{\infty} R$, temos que $\alpha \beta((C))=\alpha\left(\mathcal{C}^{\perp}\right)={ }^{\perp}\left(\mathcal{C}^{\perp}\right) \cap \mathrm{FP}_{\infty}=\mathcal{C}$. Segue por [8] Teorema 1.3 (3) que $\alpha \beta=i d$.

Como vimos anteriormente, um par de cotorção é um par de subcategorias $(\mathcal{A}, \mathcal{B})$ satisfazendo a condição $\mathcal{A}=\mathcal{B}^{\perp_{1}} \mathcal{B}={ }^{\perp_{1}} \mathcal{A}$. Nesse capítulo trabalharemos com pares de categorias $(\mathcal{A}, \mathcal{B})$ onde $\mathcal{A}=\mathcal{B}^{\perp}$ e $\mathcal{B}={ }^{\perp} \mathcal{A}$, aqui $X^{\perp}=\bigcap_{i \in \mathbb{N}} X^{\perp_{i}}$.

\subsection{Teoria tilting e cotilting}

Apresentaremos a seguir algumas generalizações para módulos infinitamente gerados de resultados que se sabia serem válidos para módulos tiltings e cotiltings finitamente gerados e que não era conhecido se o comportamento na categoria $\operatorname{Mod} R$ era o mesmo que para a categoria dos módulos finitamente gerados sobre um anel $R$.

Seguindo algumas idéias já exploradas para classes tiltings infinitamente geradas de dimensão projetiva menor ou igual a 1 e outras da teoria finitamente gerada estabelecemos uma série de lemas que definem uma boa relação entre classes tiltings.

Em primeiro lugar, observe que um par do tipo $\left({ }^{\perp} \mathcal{X}, \mathcal{X}\right)$, onde $\mathcal{X}=M^{\perp}$, é um par de torção cogerado pelo conjunto $\Omega(M)$. Assumiremos que $\Omega(M)$ é formado pelos syzygies de alguma resolução projetiva de $M$, isso garante que $\Omega(M)$ seja de fato um conjunto. Não faz diferença qual resolução tomamos, pois duas resoluções projetivas quaisquer de um mesmo módulo serão sempre homotópicas no sentido homológico e, conseqüentemente, os funtores $\operatorname{Ext}^{n}(M,-)$ não dependerão da escolha da resolução projetiva.

O resultado a seguir é uma generalização do caso finitamente gerado (veja [19]). Provamos que para a $T$-corresolução do $R$-módulo regular $R$ a menor subcategoria em $\operatorname{Mod} R$ fechada para somandos, coprodutos e extensões contendo seus módulos é AddT. Esse resultado é, de certa forma, uma versão fraca para o caso infinito do teorema de caracterização de módulos tiltings em termos do número de somandos indecomponíveis. 
Lema 2.2.1 Seja T um R-módulo r-tilting, então existe uma seqüência exata

$$
0 \longrightarrow R \stackrel{f_{0}}{\longrightarrow} T_{0} \stackrel{f_{1}}{\longrightarrow} \cdots \stackrel{f_{k}}{\longrightarrow} T_{k} \longrightarrow 0
$$

com $T_{i} \in \operatorname{Add} T$ tal que

(a) $k \leq r$

(b) cada $f_{i}$ é a composição de coker $\left(f_{i-1}\right)$ com uma $T^{\perp}$-pré-envolvente especial do conúcleo de $f_{i-1}$.

(c) $\operatorname{Add}\left(\coprod_{i=1}^{r} T_{i}\right)=\operatorname{Add} T$.

Prova: a) Seja $(\mathcal{A}, \mathcal{B})$ o par de cotorção cogerado por $\Omega(T)$, então $\mathcal{B}$ é uma classe fechada para conúcleos de monomorfismos, contém todos os $R$-módulos injetivos e é fechada para somandos diretos e extensões. Pela Proposição 1.4.3, todo $R$ módulo admite uma $\mathcal{B}$-pré-envolvente especial e, além disso, $\mathcal{B}=T^{\perp}$ e $\mathcal{A}={ }^{\perp} \mathcal{B}$. Considere $\left(X_{0}, f_{0}\right)$ uma $\mathcal{B}$-pré-envolvente especial do módulo regular $R$. Então $f_{0}$ é um monomorfismo. Como $\operatorname{Coker}\left(f_{0}\right) \in \mathcal{A}, \mathcal{A}$ é fechada para extensões e tanto $R$ quanto $\operatorname{Coker}\left(f_{0}\right)$ estão em $\mathcal{A}$, então $X_{0} \in \mathcal{A} \cap \mathcal{B}$ e como $T$ é tilting, segue que $X_{0} \in$ AddT.

Considerando $K_{0}=\operatorname{Coker}\left(f_{0}\right)$ e $\left(X_{1}, f_{1}^{\prime}\right)$ uma $\mathcal{B}$-pré-envolvente especial de $K_{0}$ e $K_{1}=\operatorname{Coker}\left(f_{1}^{\prime}\right)$, então $K_{1} \in \mathcal{A}$, portanto $X_{1} \in \mathcal{A} \cap \mathcal{B}=\operatorname{Add} T$. Fazendo por indução $f_{i}=f_{i}^{\prime} \operatorname{coker}\left(f_{i-1}\right)$ e $K_{i}=\operatorname{Coker}\left(f_{i}\right)$, e seguindo o argumento, teremos uma seqüência exata

$$
0 \longrightarrow R \stackrel{f_{0}}{\longrightarrow} X_{0} \stackrel{f_{1}}{\longrightarrow} \cdots \stackrel{f_{r-1}}{\longrightarrow} X_{r-1} \longrightarrow K_{r-1} \longrightarrow 0
$$

com $K_{r-1} \in \mathcal{A}$.

Como $\operatorname{Ext}^{i}\left(T, K_{r-1}\right) \cong \operatorname{Ext}^{i+r}(T, R)$ pelo Lema de Dimension Shifting $1.5 .8 \mathrm{e}$ $\operatorname{Ext}^{i+r}(T, R)=0$, pois $\operatorname{pd}(T)=r$, temos que $\operatorname{Ext}^{i}\left(T, K_{r-1}\right)=0$. Portanto $X_{r}=$ $K_{r-1} \in \mathcal{B}$. Dessa forma $X_{i} \in \mathcal{A} \cap \mathcal{B}=\operatorname{Add} T$ para todos $i$ em $\{0,1,2, \ldots, r\}$. Assim temos a seqüência exata procurada. 
b) Obviamente pela definição das $f_{i}$ 's acima, cada $f_{i}$ é a composição de $f_{i}^{\prime}$ com o epimorfismo coker $\left(f_{i-1}\right)$ para todo $i \in\{1,2, \ldots, r-1\}$ e $f_{0}$ é uma $\mathcal{B}$-pré-envolvente especial ${ }^{1}$ do módulo $R$. rado.

c) Vejamos agora que $\operatorname{Add}\left(\coprod_{i=0}^{r} T_{i}\right)=\operatorname{Add} T$ também no caso não finitamente ge-

Considere $\mathcal{X}=T^{\perp} \subseteq \operatorname{Mod} R$, então $\mathcal{X}$ é fechada para conúcleos de monomorfismos. Sendo $T$ um módulo tilting $\mathcal{X} \cap^{\perp} \mathcal{X}=\operatorname{Add} T$ e portanto, fechada para coprodutos. Também sabemos que $\coprod_{i=0}^{r} T_{i} \subseteq \operatorname{Add} T$. Então $\operatorname{Add}\left(\coprod_{i=0}^{r} T_{i}\right) \subseteq \operatorname{Add} T$.

Falta verificar que $\mathcal{X} \cap^{\perp} \mathcal{X} \subseteq \operatorname{Add}\left(\coprod_{i=0}^{r} T_{i}\right)$. Para isso seja $X \in \mathcal{X} \cap^{\perp} \mathcal{X}$ e considere a resolução

$$
0 \longrightarrow K_{r} \longrightarrow M_{r} \longrightarrow \cdots \longrightarrow M_{1} \longrightarrow M_{0} \longrightarrow X \longrightarrow 0
$$

$\operatorname{com} K_{r} \in\left(\coprod_{i=0}^{r} T_{i}\right)^{\perp}$ e $M_{i} \in \operatorname{Add}\left(\coprod_{i=0}^{r} T_{i}\right)$, que existe pelo lema 2.1.4.

Como $X \in^{\perp} \mathcal{X}$, então $\operatorname{pd} X \leq r$ pelo Lema 2.1.3, logo $\operatorname{Ext}^{r+1}\left(X, K_{r}\right)=0$. Pelo Lema de Dimension Shifting 1.5.8,

$$
\operatorname{Ext}^{1}\left(K_{r-1}, K_{r}\right) \cong \operatorname{Ext}^{r+1}\left(X, K_{r}\right)
$$

então $\operatorname{Ext}^{1}\left(K_{r-1}, K_{r}\right)=0$ Portanto a seqüência

$$
0 \longrightarrow K_{r} \longrightarrow M_{r} \longrightarrow K_{r-1} \longrightarrow 0
$$

cinde. Então $K_{r} \in \operatorname{Add}\left(\coprod_{i=0}^{n} T_{i}\right) \subseteq\left(\coprod_{i=0}^{n} T_{i}\right)^{\perp}$. Também obtemos que $K_{0} \in\left(\coprod_{i=0}^{n} T_{i}\right)^{\perp}$ pois $\left(\coprod_{i=0}^{r} T_{i}\right)^{\perp}$ é fechado para conúcleos de monomorfismos. Assim $\operatorname{Ext}^{1}\left(X, K_{0}\right)=0$ e portanto, $X \in \operatorname{Add}\left(\coprod_{i=0}^{r} T_{i}\right)$

\footnotetext{
${ }^{1}$ estamos usando aqui a palavra $\mathcal{B}$-pré-envolvente especial para designar o monomorfismo da $\mathcal{B}$-pré-envolvente especial $\left(X_{0}, f_{0}\right)$.
} 
Seqüências como as do Lema 2.2.1 acima, serão denominadas de $T$-corresolução. Se nessa seqüência todos os $T_{i}$ forem finitamente gerados, então chamaremos essa resolução de $T$-corresolução finitamente gerada.

O resultado acima se restrito ao caso finitamente gerado sobre uma álgebra de Artin, teremos a noção de $T$-corresolução finitamente gerada (veja [18] versão dual do Lema 1). O próximo corolário nos garante essa afirmação.

Corolário 2.2.2 Se T é um módulo tilting finitamente gerado sobre uma álgebra de Artin, então, com a notação acima, $T_{i} \in \operatorname{add} T e \operatorname{add} T=\operatorname{add}\left(\coprod_{i=1}^{n} T_{i}\right)$.

Prova: Por [12], $T^{\perp} \cap \bmod R$ é uma subcategoria pré-envolvente de $\bmod R$, portanto todas as pré-envolventes usadas na demonstração acima podem ser tomadas finitamente geradas, logo cada $T_{i}$ é finitamente gerado, portanto estão em add $T$.

Lembrado que a terminologia usada em [12] para subcategoria pré-envolvente é subcategoria covariantemente finitas.

Corolário 2.2.3 Seja T um módulo tilting e considere uma corresolução

$$
0 \longrightarrow R \longrightarrow T_{0} \longrightarrow T_{1} \longrightarrow \cdots \longrightarrow T_{r} \longrightarrow 0 .
$$

Se $T^{\prime}=\coprod_{i=0}^{r} T_{i}$, então $T^{\perp}=\left(T^{\prime}\right)^{\perp}$.

Prova: Conseqüência imediata de 2.2.1.

O lema abaixo demonstra a versão dual para módulos cotiltings do Lema 2.2.1. Veja em [19] Lemas 1.3 e 1.4 uma versão desse resultado para módulos cotiltings injetivos puros.

Lema 2.2.4 Seja $C$ um $R$-módulo n-cotilting. Então existe uma seqüência exata

$$
0 \longrightarrow C_{k} \stackrel{f_{k}}{\longrightarrow} \cdots \longrightarrow C_{1} \stackrel{f_{1}}{\longrightarrow} C_{0} \stackrel{f_{0}}{\longrightarrow} Q \longrightarrow 0,
$$

onde $Q$ é um cogerador injetivo, tal que 
a) $k \leq n$;

b) Cada $f_{i}$ é uma ${ }^{\perp} C$-pré-cobertura especial de sua imagem;

c) $\operatorname{Prod}\left(\coprod_{i=0}^{k} C_{i}\right)=\operatorname{Prod}(C)$;

Prova: Como $C$ é um $R$-módulo $n$-cotilting, então $\mathcal{X}={ }^{\perp} C$ é uma classe de recobrimento ${ }^{2}$. Podemos então construir por indução uma seqüência exata

$$
\cdots \longrightarrow X_{1} \stackrel{f_{1}}{\longrightarrow} X_{0} \stackrel{f_{0}}{\longrightarrow} Q \longrightarrow 0
$$

onde $\left(X_{0}, f_{0}\right)$ é uma ${ }^{\perp} C$-pré-cobertura especial de $Q$ e $\left(X_{i}, f_{i}\right)$ é também uma ${ }^{\perp} C$ pré cobertura especial de $\operatorname{Im}\left(f_{i}\right)$ para todo $i>0$. Considere a seqüência truncada

$$
0 \longrightarrow K_{n} \longrightarrow X_{n} \longrightarrow \cdots \longrightarrow X_{1} \stackrel{f_{1}}{\longrightarrow} X_{0} \stackrel{f_{0}}{\longrightarrow} Q \longrightarrow 0
$$

com $K_{i}=\operatorname{Im}\left(f_{i+1}\right)=\operatorname{Ker}\left(f_{i}\right)$. Sendo cada $\left(X_{i}, f_{i}\right)$ uma ${ }^{\perp} C$-pré-cobertura especial, $K_{i} \in \mathcal{X}^{\perp}$ para cada $i \geq 0$ e $Q \in \mathcal{X}^{\perp}$, temos que cada $X_{i} \in \mathcal{X} \cap \mathcal{X}^{\perp}$. Como $\mathcal{X} \cap \mathcal{X}^{\perp}=\operatorname{Prod}(\mathrm{C})$ por [6], então cada $X_{i} \in \operatorname{Prod}(C)$. Pelo Lema de Dimension Shifting 1.5.8, $\operatorname{Ext}^{j}\left(K_{n}, C\right) \cong \operatorname{Ext}^{j+k}(Q, C)$. Mas $\operatorname{Ext}^{j+k}(Q, C)=0$, pois id $C \leq n$. Portanto $K_{n}$ e $K_{n-1}$ pertencem a $\operatorname{Prod} C$ e por indução cada $K_{i} \in \operatorname{Prod} C$. Logo a seqüência

$$
0 \longrightarrow K_{n} \longrightarrow X_{n} \longrightarrow \cdots \longrightarrow X_{1} \stackrel{f_{1}}{\longrightarrow} X_{0} \stackrel{f_{0}}{\longrightarrow} Q \longrightarrow 0
$$

satisfaz as condições a) e b).

Sabemos que $\mathcal{X}$ é fechada para núcleos de epimorfismos e $\mathcal{X} \cap \mathcal{X}^{\perp}$ é fechada para produtos pela Proposição 2.1.5 item 2), então $\operatorname{Prod}\left(\coprod_{i-0}^{n} X_{i}\right) \subseteq \mathcal{X} \cap \mathcal{X}^{\perp}$.

Para a outra inclusão seja $X \in \mathcal{X} \cap \mathcal{X}^{\perp}$ e considere a seqüência

$$
0 \longrightarrow X \longrightarrow M_{0} \longrightarrow \cdots \longrightarrow M_{n-1} \longrightarrow K_{n} \longrightarrow 0
$$

\footnotetext{
${ }^{2}$ Veja a Proposição 1.4.4 sobre a existência de pré-coberturas.
} 
$\operatorname{com} M_{i} \in \operatorname{Prod}\left(\coprod_{i=0}^{n} X_{i}\right) \subseteq \operatorname{Prod} C$ e $K_{n} \in \mathcal{X}$. Como $X \in \mathcal{X} \cap \mathcal{X}^{\perp}, \operatorname{id} X \leq n$ e,

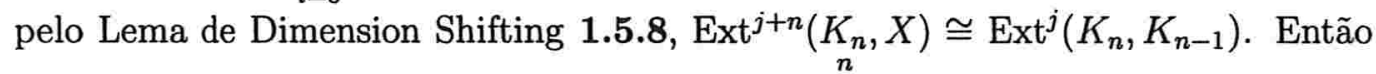
$\operatorname{Ext}^{j}\left(K_{n}, K_{n-1}\right)=0$. Logo $K_{n}$ e $K_{n-1} \in \operatorname{Prod}\left(\coprod_{i=0}^{n} X_{i}\right)$. Como $\mathcal{X}$ é fechada para núcleo de epimorfismo, por indução temos que $K_{0} \in \mathcal{X}$, então $\operatorname{Ext}^{1}\left(K_{0}, X\right)=0$. Portanto a seqüência exata

$$
0 \longrightarrow X \longrightarrow M_{0} \longrightarrow K_{0} \longrightarrow 0
$$

cinde e conseqüentemente $X \in \operatorname{Prod} \coprod_{i=0}^{n} X_{i}$. Logo $\mathcal{X} \cap \mathcal{X}^{\perp} \subseteq \operatorname{Prod} \coprod_{i=0}^{n} X_{i}$. Concluímos assim que Prod $\coprod_{i=0}^{n} X_{i}=\mathcal{X} \cap \mathcal{X}^{\perp}=\operatorname{Prod} C$ o que prova $\mathrm{c}$ ).

\subsection{Propriedades das classes tiltings e cotiltings}

Apresentaremos nessa seção uma série de lemas importantes que nos ajudarão a relacionar as classes ortogonais de dois módulos tiltings (ou cotiltings) $T$ e $N$ e associar uma $T$-corresolução do $R$-módulo $R$ com uma $N$-corresolução de $R$ (ou $T$ - e $N$-resoluções de um cogerador injetivo $Q$ no caso cotilting). Esses lemas nos fornecerão critérios mais simples para se verificar a validade de algumas condições sobre as classes ortogonais desses módulos. Mais à frente essas tais condições serão utilizadas para definirmos as chamadas seqüências tiltings e seqüências cotiltings.

Lema 2.3.1 Sejam $R$ um anel e $T$ um $R$-módulo tilting tais que $\mathcal{T}=T^{\perp}$ seja uma classe $n$-tilting de tipo finito. Então $\mathcal{T}$ é fechada para limites diretos.

Prova: Como $T$ é de tipo finito, então $T^{\perp}=\mathcal{S}^{\perp}$ para algum conjunto $\mathcal{S}$ de módulos em $\mathrm{FP}_{\infty}(R)$. Nesse caso, todos os funtores $\operatorname{Ext}^{i}\left(S,{ }_{-}\right)$comutam com limites diretos para todo $S \in \mathcal{S}$ e para todo $i \in \mathbb{N}$. Portanto $\mathcal{T}$ é fechada para limites diretos. 
Lema 2.3.2 Sejam $U$ e $T$ dois módulos tiltings em $\operatorname{Mod} R$, tais que $U \in T^{\perp}$. Então $U^{\perp} \subseteq T^{\perp}$ e $\operatorname{pd} T \leq \operatorname{pd} U$

Prova: Suponha $X \in U^{\perp}$, então existe uma seqüência exata para a cobertura projetiva de $X$.

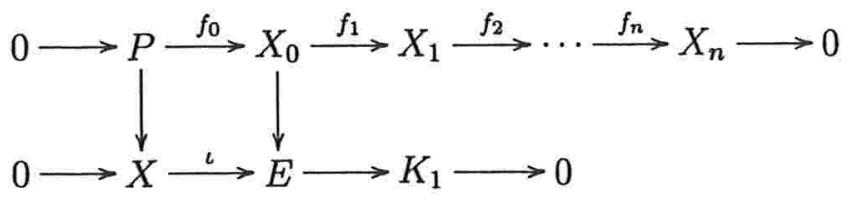

com $X_{i} \in \operatorname{Add} U$ e $K_{i} \in^{\perp}\left(U^{\perp}\right)$, onde $K_{i}=\operatorname{Coker} f_{i-1}$.

Como $X \in U^{\perp}$ e $K_{1} \in^{\perp}\left(U^{\perp}\right)$, então $\operatorname{Ext}^{i}\left(K_{1}, X\right)=0$ para todos $i \geq 1$. Em particular a seqüência

$$
0 \longrightarrow X \longrightarrow E \longrightarrow K_{1} \longrightarrow 0
$$

cinde. Então existe $j: E \rightarrow X$ tal que $j \circ \iota=\mathrm{id} X$. Mas nesse caso, $j$ é um epimorfismo e portanto existe um epimorfismo $f_{0}: X_{0} \rightarrow X$. Logo $X \in \operatorname{Gen} U$. Concluímos então que $U^{\perp} \subseteq \operatorname{Gen} U$. Como $U^{\perp} \subseteq \operatorname{Gen} U$ segue do Lema 2.1.4 que existe um seqüência exata

$$
0 \longrightarrow K \longrightarrow U^{\prime} \stackrel{l}{\longrightarrow} X \longrightarrow 0
$$

com $U^{\prime} \in \operatorname{Add} U, K \in U^{\perp}$ e, mais que isso, $U^{\prime}$ é uma $\operatorname{Add} U$-pré-cobertura. Assim na seqüência exata

$$
\operatorname{Hom}\left(U, U^{\prime}\right) \stackrel{l_{*}}{\longrightarrow} \operatorname{Hom}(U, X) \longrightarrow \operatorname{Ext}^{1}(U, K) \longrightarrow \operatorname{Ext}^{1}\left(U, U^{\prime}\right) \longrightarrow \operatorname{Ext}^{1}(U, X)
$$

$l_{*}$ é sobrejetora e, portanto, $\operatorname{Ext}^{1}(U, K)=0$. Como para $i>1 \operatorname{Ext}^{i}(U, X) \cong$ $\operatorname{Ext}^{i}\left(U, U^{\prime}\right)$ e $\operatorname{Ext}^{i}\left(U, U^{\prime}\right)=0$, pois $U$ é tilting, então $\operatorname{Ext}^{i}(U, K)=0$. Logo $K \in U^{\perp}$.

Seguindo esse argumento teremos, por indução finita, uma seqüência exata

$$
\cdots \longrightarrow U_{1} \stackrel{f_{1}}{\longrightarrow} U_{0} \stackrel{f_{0}}{\longrightarrow} X \longrightarrow 0
$$


Com $U_{i} \in \operatorname{Add} U$.

Seja $K_{i}$ o núcleo de $f_{i}$. Se $\operatorname{pd}(T)=r$, da seqüência exata 2.2 acima, temos $\operatorname{Ext}^{i}(T, X) \cong \operatorname{Ext}^{r+i}\left(T, K_{r}\right)=0$ e portanto, $X \in T^{\perp}$. Logo $U^{\perp} \subseteq T^{\perp}$ e então ${ }^{\perp}\left(U^{\perp}\right) \supseteq^{\perp}\left(T^{\perp}\right)$. Dessa forma $X \in^{\perp}\left(T^{\perp}\right)$ e isso implicará que $X \in \in^{\perp}\left(U^{\perp}\right)$. Portanto $\operatorname{pd}(X) \leq \operatorname{pd}(U)$. Em particular $\operatorname{pd}(T) \leq \operatorname{pd}(U)$.

$\mathrm{O}$ resultado acima ainda permanece válido quando trabalhamos na categoria de módulos finitamente gerados sobre uma álgebra de Artin $\Lambda$ e considerarmos as classes $T^{\perp} \mathrm{e}^{\perp}\left(T^{\perp}\right)$ restritas a $\bmod \Lambda$. Isso porque a classe $T^{\perp}$ restrita a $\bmod \Lambda$ ainda é uma classe pré-envolvente. Para maiores detalhes veja [12].

O seguinte lema é uma generalização para $\operatorname{Mod} R$ de Lema 2.2 de [19].

Lema 2.3.3 Sejam $U$ e $T$ dois módulos cotiltings em $\operatorname{Mod} R$, tais que $U \in^{\perp} T$. Então ${ }^{\perp} U \subseteq{ }^{\perp} T$ e id $T \leq \operatorname{id} U$.

Prova: Seja $X \in \in^{\perp} U$. Como $U$ é um $R$-módulo cotilting ${ }^{\perp} U \subseteq \operatorname{Cogen}(U)$. Portanto $\operatorname{Hom}(X, U) \neq 0$. Pelo Lema 2.1.4, existe uma seqüência

$$
0 \longrightarrow X \longrightarrow U_{0} \longrightarrow L \longrightarrow 0
$$

com $U_{0} \in \operatorname{Prod} U$ uma $\operatorname{Prod} U$-pré-envolvente e $L \in^{\perp} U$. Como $U_{0}$ é uma $\operatorname{Prod} U$ pré-envolvente de $X$, aplicando o funtor $\operatorname{Hom}(-, U)$ temos a seqüência exata longa de cohomologia

$$
\operatorname{Hom}\left(U_{0}, U\right) \stackrel{l}{\longrightarrow} \operatorname{Hom}(X, U) \longrightarrow \operatorname{Ext}^{1}(L, U) \longrightarrow \operatorname{Ext}^{1}\left(U_{0}, U\right) \longrightarrow \operatorname{Ext}^{1}(X, U)
$$

com $l$ é sobrejetora e $\operatorname{Ext}^{j}\left(U_{0}, U\right)=0$ para todo $j>0$. Logo $\operatorname{Ext}^{1}(L, U)=0$ e para todo $j>1 \operatorname{Ext}^{j}(L, U) \cong \operatorname{Ext}^{j-1}(X, U)=0$, pois $X \in^{\perp} U$. Portanto $L \in^{\perp} U$. Repetindo o argumento para $X_{1}=L$ temos $U_{1}$ nas mesmas condições acima, e portanto um $L_{2} \in^{\perp} U$. Seguindo por indução finita teremos uma seqüência exata

$$
0 \longrightarrow X \longrightarrow U_{0} \longrightarrow U_{1} \longrightarrow \cdots
$$


$\operatorname{com} U_{i} \in \operatorname{Prod} U$.

Como $T$ é $n$-cotilting para algum $n \in \mathbb{N}$, obtemos da seqüência acima que $\operatorname{Ext}^{j}(X, T) \cong \operatorname{Ext}^{j+n}\left(L_{n}, T\right)=0$. Portanto $X \in^{\perp} T$ e então ${ }^{\perp} U \subseteq^{\perp} T$.

Como ${ }^{\perp} U \subseteq{ }^{\perp} T$, então $\left({ }^{\perp} U\right)^{\perp} \subseteq\left({ }^{\perp} T\right)^{\perp}$. Segue pelo Lema 2.1.3 b) que idT $\leq \operatorname{id} U$.

Lema 2.3.4 Sejam $U$ e $T$ dois módulos tiltings em $\operatorname{Mod} R$, tais que $U \in T^{\perp}$. Assuma que $\operatorname{pd}(U)=r$ para algum $r \in \mathbb{N}$.

Seja

$$
0 \longrightarrow R \stackrel{\gamma}{\longrightarrow} T_{0} \stackrel{f_{0}}{\longrightarrow} T_{1} \stackrel{f_{1}}{\longrightarrow} \cdots \longrightarrow T_{s} \longrightarrow 0
$$

uma $T$-corresolução do módulo regular $R$. Então $s \leq r$ e existe uma $U$-corresolução

$$
0 \longrightarrow R \stackrel{\delta}{\longrightarrow} U_{0} \stackrel{g_{0}}{\longrightarrow} U_{1} \stackrel{g_{1}}{\longrightarrow} \cdots \longrightarrow U_{r} \longrightarrow 0
$$

do módulo regular $R$, comutando diagrama

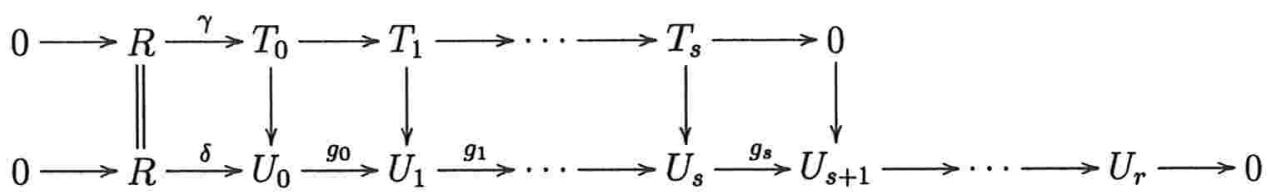

e com as seguintes propriedades:

(i) $\left(U_{0}, \delta\right)$ é uma $U^{\perp}$-pré-envolvente especial de $R$. Para todo $i>1,\left(U_{i}, \overline{g_{i}}\right)$ é uma $U^{\perp}$-pré-envolvente especial de $\operatorname{Coker}\left(g_{i-1}\right) e\left(U_{1}, \bar{g}_{0}\right)$ é uma $U^{\perp}$-pré-envolvente especial de $\operatorname{Coker}(\delta)$. Para cada $i>0, g_{i}=\bar{g}_{i} \circ \operatorname{coker}\left(g_{i-1}\right)$ e $g_{0}=\bar{g}_{0} \circ \operatorname{coker}(\gamma)$;

(ii) As aplicações verticais são $U^{\perp}$-pré-envolventes injetoras ou são nulas; 
Prova: Seja

$$
0 \longrightarrow R \stackrel{\lambda}{\longrightarrow} \widetilde{U}_{0} \stackrel{g_{1}}{\longrightarrow} \widetilde{U}_{1} \stackrel{g_{2}}{\longrightarrow} \widetilde{U}_{2} \stackrel{g_{3}}{\longrightarrow} \widetilde{U}_{3} \longrightarrow \cdots \stackrel{g_{r}}{\longrightarrow} \widetilde{U}_{r} \longrightarrow 0
$$

uma $U$-corresolução de $R$, que sabemos existir pelo Lema 2.2.1, e seja $K_{0}=\operatorname{Coker}(\lambda)$. Existe um diagrama

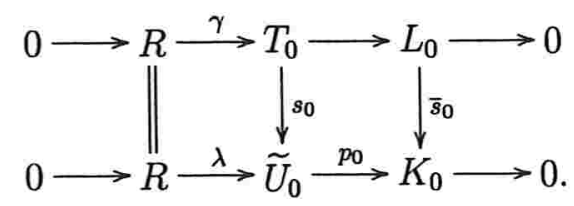

comutativo, pois $U^{\perp} \subseteq T^{\perp}$ e $\left(T_{0}, \gamma\right)$ é uma $T^{\perp}$-pré-envolvente especial de $R$. Assim, como $\widetilde{U}_{0} \in T^{\perp}$, existe $s_{0}: T_{0} \rightarrow \widetilde{U}_{0}$ tal que $\lambda=s_{0} \circ \gamma$. Passando ao conúcleo temos $\bar{s}_{0}: L_{0} \rightarrow K_{0}$ comutando o diagrama acima.

Seja $L_{i}=\operatorname{Coker}\left(f_{i}\right)$ e $K_{i}=\operatorname{Coker}\left(g_{i}\right)$, onde os homomorfismos $f_{i}$ e $g_{i}$ são os definidos pelas corresoluções acima. Então temos por indução cada $i>0$ um diagrama

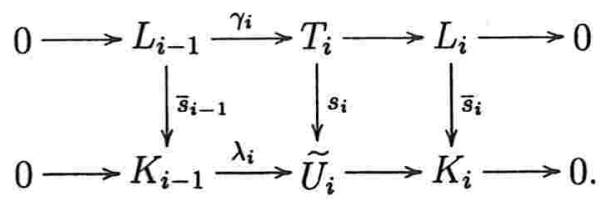

Como anteriormente o par $\left(T_{i}, f_{i}\right)$ é uma $T^{\perp}$-pré-envolvente especial de $L_{i-1}$ e como $\widetilde{U}_{i} \in T^{\perp}$, existe $s_{i}: T_{i} \rightarrow \widetilde{U}_{i}$ tal que $\lambda_{i} \bar{s}_{i-1}=s_{i} \circ \gamma_{i}$.

Como $U^{\perp} \subseteq T^{\perp}$, então $s \leq r$.

Concatenando os diagramas obtidos acima conseguimos montar o diagrama

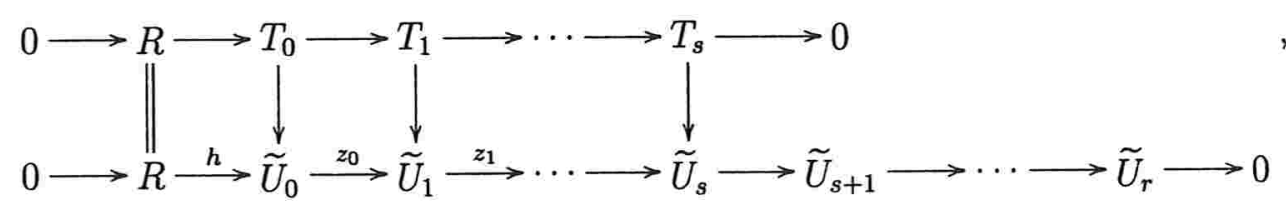

satisfazendo a condição (i) do enunciado do lema. Vamos ajustá-lo de modo a obter um diagrama satisfazendo também as condições (ii) e (iii) do enunciado acima. 
Seja $\left(\bar{U}_{0}, j_{0}\right)$ uma $U^{\perp}$-pré-envolvente especial de $L_{0}$. Primeiro observemos que $L_{0} \in^{\perp}\left(T^{\perp}\right) \subset^{\perp}\left(U^{\perp}\right)$. Como $\operatorname{Coker}\left(j_{0}\right) \in^{\perp}\left(U^{\perp}\right)$, que por sua vez é fechada para extensões, então $\bar{U}_{0} \in U^{\perp} \cap^{\perp}\left(U^{\perp}\right)=\operatorname{Add} U$. Logo conseguimos o diagrama comutativo

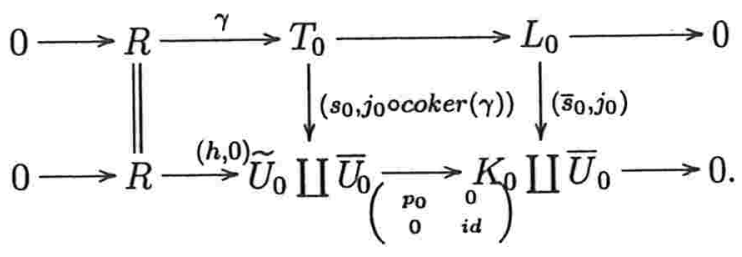

O morfismo $\left(\bar{s}, j_{0}\right)$ é um monomorfismo, pois $j_{0}$ o é. Pelo Lema dos Cinco (Five Lema veja [36] Lema 6.15) $\left(s_{0}, j_{0} \circ \operatorname{coker}(\gamma)\right)$ é monomorfismo.

Provaremos agora que o $\operatorname{par}\left(\widetilde{U}_{0} \amalg \bar{U}_{0},\left(s_{0}, j_{0}\right.\right.$ ocoker $\left.\left.(\gamma)\right)\right)$ é uma $U^{\perp}$-pré-envolvente especial de $T_{0}$.

De fato, seja $l: T_{0} \rightarrow M$ com $M \in U^{\perp}$ um morfismo qualquer. Consideremos a composição $l \circ \gamma: R \rightarrow M$. Como $M \in U^{\perp}$, então $l \circ \gamma$ se fatora por $\lambda$. Então existe $i_{\widetilde{U}_{0}}: \widetilde{U}_{0} \rightarrow M$ tal que $l \circ \gamma=i_{\widetilde{U}_{0}} \circ \lambda$. Sendo $\lambda=s_{0} \circ \gamma$ temos que $l \circ \gamma=i_{\widetilde{U}_{0}} \circ s_{0} \circ \gamma$ e portanto $\left(l-i_{\widetilde{U}_{0}} \circ s_{0}\right) \circ \gamma=0$. Pela propriedade dos conúcleos, $\left(l-i_{\widetilde{U}_{0}} \circ s_{0}\right)$ se fatora por $L_{0}$, ou seja, existe $h_{0}: L_{0} \rightarrow M$ tal que $\left(l-i_{\widetilde{U}_{0}} \circ s_{0}\right)=h_{0} \circ \operatorname{coker}(\gamma)$. Como $\left(\bar{U}_{0}, j_{0}\right)$ uma $U^{\perp}$-pré-envolvente especial de $L_{0}$, existe $b_{0}: \bar{U}_{0} \rightarrow M$ tal que $h_{0}=b_{0} \circ j_{0}$. Logo

$$
l=\left(i_{\widetilde{U}_{0}} \circ s_{0}+b_{0} \circ j_{0} \circ \operatorname{coker}(\gamma)\right)=\left(\begin{array}{ll}
i_{\widetilde{U}_{0}}, & b_{0}
\end{array}\right) \circ\left(\begin{array}{c}
s_{0} \\
j_{0} \circ \operatorname{coker}(\gamma)
\end{array}\right)
$$

Isso implica que $l$ se fatora por $U^{\perp}$ e portanto $\left(\widetilde{U}_{0} \amalg \bar{U}_{0},\left(s_{0}, j_{0} \circ \operatorname{coker}(\gamma)\right)\right)$ é uma $U^{\perp}$-pré-envolvente especial de $T_{0}$.

Observe que o $\operatorname{par}\left(\widetilde{U}_{0} \amalg \bar{U}_{0},(h, 0)\right)$, ainda continua sendo uma $U^{\perp}$-pré-envolvente especial de $R$, pois $\operatorname{Ker}(h, 0) \cong \operatorname{Ker}(h) \amalg U_{0}$ que pertence a ${ }^{\perp}\left(U^{\perp}\right)$.

Faremos agora o passo indutivo da construção dos diagramas.

Considere o conúcleo do passo anterior. Então podemos construir o seguinte diagrama. 


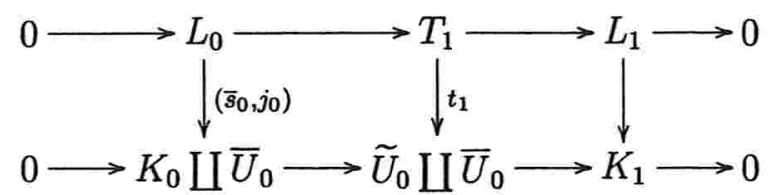

Note que para o morfismo $t:\left(K_{0} \amalg \bar{U}_{0}\right) \rightarrow \widetilde{U}_{0} \amalg \bar{U}_{0}$ no diagrama acima, o par $\left(\widetilde{U}_{0} \amalg \bar{U}_{0}, t\right)$ é uma $U^{\perp}$ - pré-envolvente especial de $K_{0} \amalg \bar{U}_{0}$.

Considere um monomorfismo $j_{1}: L_{1} \longrightarrow \bar{U}_{1}$ tal que o par $\left(\bar{U}_{1}, j_{1}\right)$ seja uma $U^{\perp}$-pré-envolvente especial de $L_{1}$. Então $L_{1}$ e $\operatorname{Coker}\left(j_{1}\right) \in \perp^{\perp}\left(U^{\perp}\right)$. Como ${ }^{\perp}\left(U^{\perp}\right)$ é fechada para extensões temos que $\bar{U}_{1} \in^{\perp}\left(U^{\perp}\right)$, logo $\bar{U}_{1} \in^{\perp}\left(U^{\perp}\right) \cap U^{\perp}=\operatorname{Add} U$.

Como no caso anterior construímos o diagrama

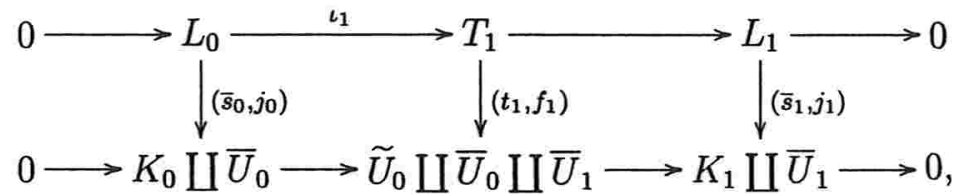

onde $f_{1}=j_{1} \circ \operatorname{coker}\left(\iota_{1}\right)$.

Um argumento similar mostra que $\left(\widetilde{U}_{1} \amalg \bar{U}_{0} \amalg \bar{U}_{1},\left(t_{1}, f_{1}\right)\right)$ é uma $U^{\perp}$-pré-envolvente de $T_{1}$.

Como podemos ver no diagrama essa situação se repetirá para todos $i \in\{1,2, \ldots, r\}$ e por indução teremos

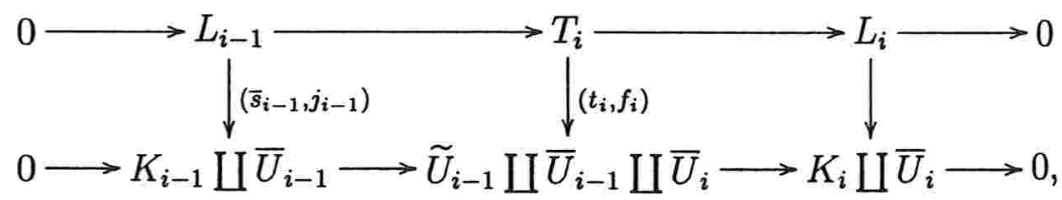

com $\left(\widetilde{U}_{i-1} \amalg \bar{U}_{i-1} \amalg \bar{U}_{i},\left(t_{i}, f_{i}\right)\right.$ uma $U^{\perp}$-pré-envolvente de $T_{i}$.

Apresentaremos a seguir a versão correspondente para módulos cotiltings.

Lema 2.3.5 Sejam $U$ e $T$ dois módulos cotiltings em $\operatorname{Mod} R$, tais que $U \in^{\perp} T$. Assuma que $\operatorname{id}(U)=r$ para algum $r \in \mathbb{N}$. 
Seja

$$
0 \longrightarrow T_{s} \stackrel{f_{s}}{\longrightarrow} \cdots \stackrel{f_{1}}{\longrightarrow} T_{0} \stackrel{f_{0}}{\longrightarrow} Q \longrightarrow 0
$$

uma T-corresolução de um cogerador injetivo $Q$. Então $s \leq r$ e existe uma $U$ resolução do cogerador injetivo $Q$

$$
0 \longrightarrow U_{r} \stackrel{g_{r}}{\longrightarrow} \cdots \stackrel{g_{1}}{\longrightarrow} U_{0} \stackrel{g_{0}}{\longrightarrow} Q \longrightarrow 0
$$

comutando o diagrama

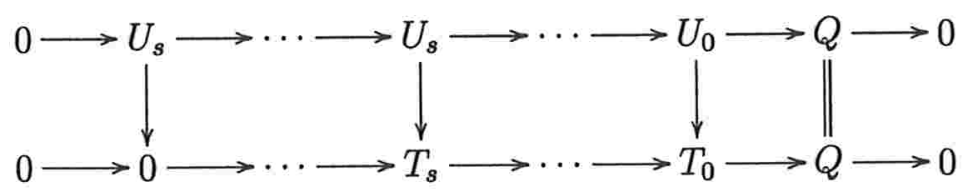

e com as seguintes propriedades:

(i) Todos os pares $\left(U_{i}, g_{i}\right)$ são ${ }^{\lfloor} U$-pré-coberturas especiais da imagem de $g_{i}$;

(ii) As aplicações verticais são ${ }^{\perp} U$-pré coberturas sobrejetoras. ${ }^{3}$

Prova: Seja

$$
0 \longrightarrow \widetilde{U}_{r} \longrightarrow \cdots \longrightarrow \widetilde{U}_{1} \stackrel{z_{1}}{\longrightarrow} \widetilde{U}_{0} \stackrel{z_{0}}{\longrightarrow} Q \longrightarrow 0
$$

uma $U$-corresolução de um cogerador injetivo $Q$. Seja $L_{1}=\operatorname{Ker}\left(z_{0}\right)$ e para cada $0 \leq i \leq r-1 L_{i}=\operatorname{Ker}\left(z_{i}\right)$ e $K_{i+1}=\operatorname{Ker}\left(f_{i}\right)$. Sendo que $\widetilde{U}_{0} \in^{\perp} T$, existe $h: \widetilde{U}_{0} \rightarrow T_{0}$ e $q_{1}: L_{1} \rightarrow K_{1}$ tal que o diagrama abaixo comuta.

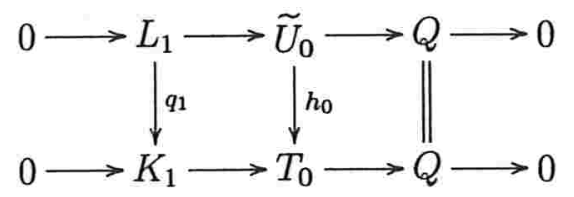

Utilizando-se do mesmo argumento podemos construir o diagrama

\footnotetext{
${ }^{3}$ Novamente, estamos nos referindo apenas ao morfismo da pré-cobertura.
} 


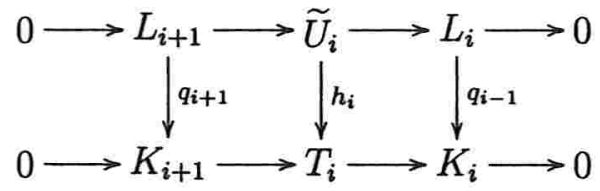

para cada $i$. Como no caso tilting esses diagramas podem ser concatenados de forma a satisfazer a condição $(i)$. Vamos modificá-los de forma a satisfazer as condições (i) e (ii) simultaneamente.

Seja $\left(\bar{U}_{0}, p_{0}\right)$ uma ${ }^{\perp} U$-pré-cobertura especial de $K_{1}$. Primeiro observemos que $K_{1} \in\left({ }^{\perp} T\right)^{\perp} \subseteq\left({ }^{\perp} U\right)^{\perp}$. Como $\operatorname{Ker}\left(p_{0}\right) \in\left({ }^{\perp} U\right)^{\perp}$, então $\bar{U}_{0} \in\left({ }^{\perp} U\right)^{\perp} \cap^{\perp} U=\operatorname{Prod} U$. Então obtemos um diagrama

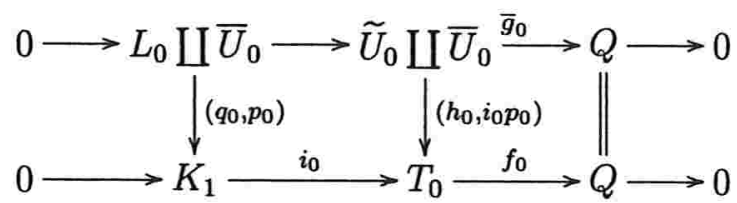

onde $\bar{g}_{0}=\left(g_{0}, 0\right)$. sendo que $p_{0}$ é um epimorfismo, então $\left(q_{0}, p_{0}\right)$ é necessariamente um epimorfismo. Sendo $\operatorname{id}_{Q}$ também sobrejetora, novamente pelo Lema do Cinco (Five Lema [36] Lema 6.15), $\left(h_{0}, i_{0} p_{0}\right)$ é sobrejetora.

Mostraremos que $\left(\widetilde{U}_{0} \amalg \bar{U}_{0},\left(h_{0}, \iota_{0} p_{0}\right)\right.$ é uma ${ }^{\perp} U$-pré-cobertura de $T_{0}$.

De fato, seja $b: X \rightarrow T_{0}$ um morfismo com $X \in^{\perp} U$, então $f_{0} b$ se fatora por $\widetilde{U}_{0}$. Logo existe $l: X \rightarrow \widetilde{U}_{0}$ tal que $f_{0} b=g_{0} l$. Temos assim uma aplicação

$$
\left(\begin{array}{l}
l \\
0
\end{array}\right): X \rightarrow \widetilde{U}_{0} \coprod \bar{U}_{0}
$$

satisfazendo a igualdade $\bar{g}_{0} \circ\left(\begin{array}{l}l \\ 0\end{array}\right)=f_{0} b$ e tal que

$$
f_{0}\left(h_{0}, \iota_{0} p_{0}\right) \circ\left(\begin{array}{l}
l \\
0
\end{array}\right)=\bar{g}_{0} \circ\left(\begin{array}{l}
l \\
0
\end{array}\right)=g_{0} l=f_{0} b .
$$

Logo $f_{0}\left[\left(h_{0}, \iota_{0} p_{0}\right) \circ\left(\begin{array}{l}l \\ 0\end{array}\right)-b\right]=0$ e portanto $\operatorname{Im}\left[\left(h_{0}, \iota_{0} p_{0}\right) \circ\left(\begin{array}{l}l \\ 0\end{array}\right)-b\right] \subset \operatorname{Ker} f_{0}=K_{1}$. Sendo que $\left(\bar{U}_{0}, p_{0}\right)$ é uma ${ }^{\perp} U$-pré cobertura especial $K_{1}$ existe $s: X \rightarrow \bar{U}_{0}$ tal que 


$$
\begin{aligned}
\iota_{0} p_{0} s=\left(h_{0}, \iota_{0} p_{0}\right) & \circ\left(\begin{array}{l}
l \\
0
\end{array}\right)-b, \text { portanto } \\
\qquad & =\iota_{0} p_{0} s-\left(h_{0}, \iota_{0} p_{0}\right) \circ\left(\begin{array}{l}
l \\
0
\end{array}\right)=\left(\iota_{0} p_{0}, h_{0}\right) \circ\left(\begin{array}{c}
s \\
-l
\end{array}\right) .
\end{aligned}
$$

Logo $b$ fatora-se através $\left(h_{0}, \iota_{0} h_{0}\right)$. Segue que $\left(\widetilde{U}_{0} \amalg \bar{U}_{0},\left(h_{0}, \iota_{0} p_{0}\right)\right)$ é uma ${ }^{\perp} U$-pré cobertura sobrejetora.

Por indução construímos o diagrama

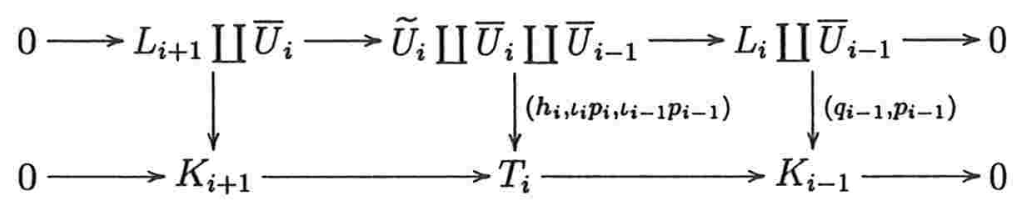

Com o mesmo argumento utilizado acima podemos mostrar que

$$
\left(\widetilde{U}_{i} \coprod \bar{U}_{i} \coprod \bar{U}_{i-1},\left(h_{i}, \iota_{i} p_{i}, \iota_{i-1} p_{i-1}\right)\right)
$$

é uma ${ }^{\perp} U$-pré cobertura sobrejetora de $T_{i}$.

Lema 2.3.6 Sejam $U$ e $T$ módulos cotiltings em $\bmod \Lambda$ para uma álgebra de Artin $\Lambda$. Se $U \in^{\perp} T \cap \bmod \Lambda$, então $T \in \operatorname{gen}(U)$

Prova: Como ${ }^{\perp} U \cap \bmod \Lambda$ é uma classe de recobrimento em $\bmod \Lambda$ (veja [12]), existe uma seqüência exata

$$
0 \longrightarrow Y \longrightarrow X \stackrel{f}{\longrightarrow} T \longrightarrow 0
$$

com $(X, f)$ uma ${ }^{\perp} U \cap \bmod \Lambda$-pré cobertura especial de $T$. Então $X \in^{\perp} U \cap \bmod \Lambda$ e $Y \in\left({ }^{\perp} U\right)^{\perp} \cap \bmod \Lambda$. Pelo Lema 2.3.3 ${ }^{\perp} U \subseteq \subseteq^{\perp} T$, portanto $\left({ }^{\perp} T\right)^{\perp} \subseteq\left({ }^{\perp} U\right)^{\perp}$. Logo $T \in\left({ }^{\perp} U\right)^{\perp} \cap \bmod \Lambda$. Sendo $\left({ }^{\perp} U\right)^{\perp} \cap \bmod \Lambda$ fechada para extensões

$$
X \in{ }^{\perp} U \cap\left({ }^{\perp} U\right)^{\perp} \cap \bmod \Lambda=\operatorname{add} U .
$$


Portanto $U$ gera $T$.

Observe que se $T$ e $U$ são módulos finitamente gerados sobre uma álgebra de Artin $\Lambda$, então $U \in^{\perp} T \cap \bmod \Lambda$ e pelo Lema 2.3.6, $U$ gera $T$, então o Lema 2.3.5 se reduz a [19] Lema 2.3, ou seja, o Lema 2.3.5 e válido para a categoria de módulos finitamente gerados.

\subsection{Módulos $n$-tiltings parciais de tipo finito}

Vamos verificar a validade de alguns resultados sobre módulos tiltings parciais finitamente gerados para a classe dos módulos tiltings de tipo finito e explorar uma certa unicidade para módulos tiltings infinitamente gerados.

Proposição 2.4.1 Sejam $N$ um R-módulo n-tilting parcial e $\mathcal{X}=N^{\perp}$, então $N$ é tilting se e somente se $\operatorname{Add} N=\mathcal{X} \cap \perp \mathcal{X}$.

Prova: Se $N$ é tilting então $\operatorname{Add} N=\mathcal{X} \cap^{\perp} \mathcal{X}$ por 2.1.4

Suponha então que $\operatorname{Add} N=\mathcal{X} \cap^{\perp} \mathcal{X}$, como $\operatorname{pd} N \leq n$, então existe uma seqüência

$$
0 \longrightarrow R \longrightarrow N_{0} \longrightarrow \cdots \longrightarrow N_{n} \longrightarrow 0
$$

com cada $N_{i} \in \mathcal{X} \cap{ }^{\mathcal{L}} \mathcal{X}$. Sabemos que ${ }^{\mathcal{X}} \mathcal{X} \subseteq \mathcal{P}_{n}$ por 2.1.3. Sendo $N$ de tipo finito $\mathcal{X}$ é fechada para somas diretas e somandos, portanto $\operatorname{Add}\left(\coprod_{i=0}^{n} N_{i}\right) \subseteq \mathcal{X} \cap \perp \mathcal{X}=\operatorname{Add} N$ e nesse caso $N_{i} \in \operatorname{Add} N$ para todo $i=0,1, \ldots, n$, logo $N$ é tilting.

A proposição abaixo, foi demonstrada recentemente por Oyvind Solberg, mas como essa demonstração foi produzida de forma independente e a utilizaremos no Capítulo 4, resolvemos apresentá-la aqui. A proposição é uma decorrência quase que imediata de [7] 2.1.

Proposição 2.4.2 Todo módulo n-tilting parcial de tipo finito pode ser completado a um n-tilting de tipo finito. 
Prova: Seja $M$ um $R$-módulo $n$-tilting de tipo finito, então $\mathcal{X}=M^{\perp}$ é fechada para limites diretos e, portanto, fechada para coprodutos.

Considere a seqüência exata

$$
0 \longrightarrow R \stackrel{f}{\longrightarrow} N \longrightarrow C \longrightarrow 0,
$$

onde $f$ é uma $\mathcal{X}$-pré-envolvente especial de $R$, então $\operatorname{Ext}^{i}(C, \mathcal{X})=0$ para todo $i \in \mathbb{N}$. Portanto $C \in^{\perp} \mathcal{X}$. Sendo ${ }^{\perp} \mathcal{X}$ fechada para extensões temos que $N \in^{\perp} \mathcal{X}$ e por [6] $4.1 \mathrm{~b}) \operatorname{pd} N \leq n$. Também sabemos que $\mathcal{X} \in \operatorname{Gen}(N)$ se $T=N \amalg M$, então $T^{\perp}=M^{\perp}$ e Gen $T=\operatorname{Gen} N$. Portanto $T^{\perp} \subset \operatorname{Gen} T$. Segue por [6] 4.4 que $T$ é $n$-tilting, e como $T^{\perp}=M^{\perp}$, então $T$ é necessariamente de tipo finito.

Além disso $\operatorname{Add} T=\mathcal{X} \cap^{\perp} \mathcal{X}$ e existe uma seqüência

$$
0 \longrightarrow R \stackrel{f_{0}}{\longrightarrow} C_{0} \stackrel{f_{1}}{\longrightarrow} \cdots \stackrel{f_{n}}{\longrightarrow} C_{n} \longrightarrow 0
$$

$\operatorname{com}\left(C_{i}, f_{i}\right)$ uma $\mathcal{X}$-pré-envolvente especial de $\operatorname{Coker}\left(f_{i-1}\right)$. Então $\prod_{i=0}^{n} C_{i}$ é $n$-tilting $\operatorname{e} \operatorname{Add}\left(\coprod_{i=0}^{n} C_{i}\right)=\operatorname{Add}(T)=\operatorname{Add} M \coprod N$.

\subsection{Módulos $n$-tiltings maximais}

Quando trabalhamos com teoria de módulos finitamente gerados, módulos tiltings são somas diretas de módulos indecomponíveis e então podemos sempre considerar um representante básico para esse módulo, isto é, um módulo sem somandos diretos repetidos. Então sua classe tilting pode ser representada através desse módulo básico. Isso entretanto no caso infinito não é tão simples. Mas existe uma outra forma de se obter unicidade com relação a uma classe tilting $\mathcal{X}$. Essa nova noção foi introduzida em [8] para módulos tiltings com dimensão projetiva menor ou igual a $1 \mathrm{em} \operatorname{Mod} R$.

Definiremos abaixo a mesma noção para módulos $n$-tiltings fazendo uma ligeira 
adaptação na definição. Substituiremos a classe Gen $T$ pela classe $T^{\perp}$, pois se $\operatorname{pd} T \leq$ 1 então as classes $T^{\perp}$ e GenT coincidem.

Sejam $R$ um anel e $M \in \operatorname{Mod} R$. Definimos o $\operatorname{spn}(M)$ como sendo a menor cardinalidade dos conjuntos de $R$-geradores de $M$ e para uma classe $n$-tilting $\mathcal{X}$ definimos $\operatorname{trank}(\mathcal{X})=\min \left\{\operatorname{spn} T / T\right.$ é $n-$ tilting e $\left.T^{\perp}=\mathcal{X}\right\}$.

Definição 2.5.1 Dizemos que um módulo $T$ é tilting maximal se $T=\coprod_{\alpha<\kappa} T_{\alpha}$, onde a familia $\left\{T_{\alpha}\right\}_{\alpha<\kappa}$ satisfaz:

1) $T_{\alpha}$ é n-tilting $\forall \alpha<\kappa$.

2) $T_{\alpha} \not T_{\beta} \operatorname{mas} T_{\alpha}^{\perp}=T_{\beta}^{\perp}$

3) $\operatorname{spn}\left(T_{\alpha}\right)=\operatorname{trank}\left(T^{\perp}\right) \forall \alpha<\kappa$

4) Se $T^{\prime}$ é um $R$-módulo tilting tal que $\left(T^{\prime}\right)^{\perp}=T^{\perp}$ e $\operatorname{spn}\left(T^{\prime}\right)=\operatorname{trank}\left(T^{\perp}\right)$, então existe $\alpha<\kappa$ tal que $T^{\prime} \cong T_{\alpha}$.

Seja $\mathcal{M}_{n}$ a classe formada por todas as classes de isomorfismos de módulos $n$ tiltings maximais e $\mathcal{I}_{n}$ a classe formada por todas as classes de isomorfismos de módulos $n$-tiltings.

Proposição 2.5.2 Seja $R$ um anel. Então a função de classes

$$
b: \mathcal{M}_{n} \rightarrow \mathcal{I}_{n}
$$

dada por $b(T)=T^{\perp}$ é uma bijeção

Prova: 1) Vamos verificar que $b$ está bem definida. Suponha $T_{1} \cong T_{2}$, então $T_{1}^{\perp}=\bigcap_{i \in \mathbb{M} \backslash\{0\}} \operatorname{Ext}^{i}\left(T_{1},-\right)=\bigcap_{i \in \mathbb{N} \backslash\{0\}} \operatorname{Ext}^{i}\left(T_{2},-\right)=T_{2}^{\perp}$. Portanto definem a mesma classe $n$-tilting.

2) $b$ é injetora. De fato, sejam $T_{1}$ e $T_{2}$ dois módulos $n$-tiltings maximais tais que $b\left(T_{1}\right)=b\left(T_{2}\right)$, ou seja, $T_{1}^{\perp}=T_{2}^{\perp}$. Como $T_{1}$ e $T_{2}$ são tiltings maximais $T_{1}=\coprod_{\alpha<\kappa_{1}} T_{\alpha}$ e $T_{2}=\coprod_{\alpha<\kappa_{2}} T_{\alpha}$. 
Como para cada $T_{\alpha}^{1},\left(T_{\alpha}^{1}\right)^{\perp}=T_{\alpha}^{\perp}$ e por hipótese, $T_{1}^{\perp}=T_{2}^{\perp}$ e $\operatorname{spn}\left(T_{\alpha}^{1}\right)=$ $\operatorname{trank}\left(T_{1}\right)=\operatorname{trank}\left(T_{2}\right)=\operatorname{spn}\left(T_{\lambda_{\alpha}}\right)$, existe $\lambda_{\alpha}$ tal que $T_{\alpha}^{1} \cong T_{\lambda_{\alpha}}^{2}$. Nesse caso $T_{\alpha}^{1}$ é um somando de $T^{2}$, e portanto $T_{1}$ é é um somando de $T^{2}$. De forma análoga $T^{2}$ é um somando de $T^{1}$. Isso prova que $b$ é injetora.

3) A função $b$ é sobrejetora. Seja $\mathcal{T}$ uma classe $n$-tilting, então existe um $R$ módulo $n$-tilting $T_{0}$ tal que $\mathcal{T}=T_{0}^{\perp}$. Escolha um representante de cada classe de equivalência de isomorfismo de $\mathcal{A}$.

Seja $\mathcal{A}$ um conjunto representativo da classe de isomorfismos dos $R$-módulos $n$ tilting $M$ tais que $M^{\perp}=T_{0}^{\perp}$ e $\operatorname{spn}(M)=\operatorname{trank}(\mathcal{T})$ e seja $T=\coprod_{M \in \mathcal{A}} M$, então $T$ é $n$-tilting e $T^{\perp}=\mathcal{T}$.

Vamos indicar o módulo $n$-tilting maximal associado a um módulo $n$-tilting de tipo finito $T$ por $T_{\max }$.

Como conseqüência, cada $n$-tilting está associado a um $n$-tilting maximal, então para cada $n$-tilting de tipo finito temos um $n$-tilting de maximal de tipo finito que está associado a uma subclasse resolvente de módulos em $\bmod R$. Portanto existe uma correspondência bijetiva entre os módulos tiltings de tipo finito e as subclasses resolventes de módulos $\bmod R$ contidas em $\mathcal{P}_{n}$.

Vamos indicar o módulo n-tilting maximal associado a um módulo n-tilting de tipo finito por $T_{\max }$.

Em particular, se $T$ e $N$ são tiltings com $\operatorname{Add} T=\operatorname{Add} N$, então os tiltings $T_{\max }$ e $N_{\max }$ são isomorfos.

Veremos no Capítulo 4 que componentes de conexão de álgebras inclinadas (tilted) estáveis à direita definem um único tilting maximal de tipo finito. 


\section{Capítulo 3}

\section{Limites Tiltings e Cotiltings}

Como já mencionamos anteriormente, a noção de módulos tiltings foi estendida para módulos de dimensão projetiva menor ou igual a $n$, para um número natural $n$, por Angeleri-Hügel e Coelho em [6]. Nesse trabalho eles exibem um exemplo de um módulo $n$-tilting infinito, partindo de um módulo finitamente gerado $M$ com $\operatorname{pd} M \leq n$ (Exemplo 2.1). A idéia é bastante simples, pois considera o módulo $n$ tilting $M^{(I)}$, ou seja, um coproduto do módulo original $M$. Entretanto, essa idéia, mesmo sendo simples, inspirou Buan e Solberg a construírem um exemplo dual para módulos cotiltings, como um somando de um produto de módulos $n$-cotiltings para os quais a dimensão projetiva é limitada. Eles construíram um limite inverso de uma seqüência de módulos cotiltings satisfazendo algumas "boas" propriedades. Esse limite inverso é um módulo injetivo puro e portanto será um somando do produto dos módulos cotiltings da sequiência. Partimos dessa idéia para tentar construir um módulo tilting como limite direto de um sistema direto de módulos tiltings satisfazendo as propriedades duais àquelas utilizadas por Buan e Solberg em [19].

Desenvolvemos esse terceiro capítulo no intuito de caracterizar seqüências de $R$-módulos tiltings (cotiltings) que podem gerar limites diretos (inversos) que ainda sejam $R$-módulo tiltings (cotiltings). Para isso, generalizamos conceitos e resultados conhecidos para módulos tiltings e cotiltings finitamente gerados para o contexto da categoria $\operatorname{Mod} R$ no capítulo anterior e agora estamos prontos para construir 
módulos tiltings e cotiltings não finitamente gerados. Algumas restrições no entanto aparecem quando tentamos fazer isso. Com a técnica aqui utilizada, os limites diretos (inversos) só são possíveis quando trabalhamos com famílias de módulos tiltings de tipo finito (cotiltings finitamente gerados). Tais limites diretos (inversos), em geral são módulos não finitamente gerados, mesmo que a família utilizada para obtê-lo seja formada por módulos $\mathrm{em} \mathrm{FP}_{\infty}(R)(\bmod R)$.

No caso cotilting os resultados estão demonstrados em [18] e [19], mas vamos apresentá-los novamente aqui para poder comparar propriedades que só funcionam no caso cotilting com as propriedades que utilizamos na construção do limite tilting.

\subsection{Construção de um sistema direto de módulos tiltings}

Nesta seção iremos construir um sistema direto de módulos tiltings de tipo finito com a propriedade de que cada morfismo do sistema dirigido de módulos seja um monomorfismo. Com isso em mente, construiremos um módulo tilting infinitamente gerado como limite direto desse sistema. Também apresentaremos a construção do sistema inverso para módulos cotiltings finitamente gerados [19].

Sejam $R$ um anel e $\left\{T^{0}, T^{1}, \ldots\right\}$ um seqüência de módulos tiltings de tipo finito tais que $\operatorname{Add} T^{i} \neq \operatorname{Add} T^{j}$ se $i \neq j$. Assuma que $T^{i+1} \in\left(T^{i}\right)^{\perp}$ e $\operatorname{pd} T^{i} \leq n \forall i \in \mathbb{N}$.

Pelo Lema 2.3.2 $\left(T^{i+1}\right)^{\perp} \subseteq\left(T^{i}\right)^{\perp}$.

Sendo cada $T^{i}$ um $R$-módulo tilting, usando indução e o Lema 2.3.4 conseguimos 
construir o diagrama

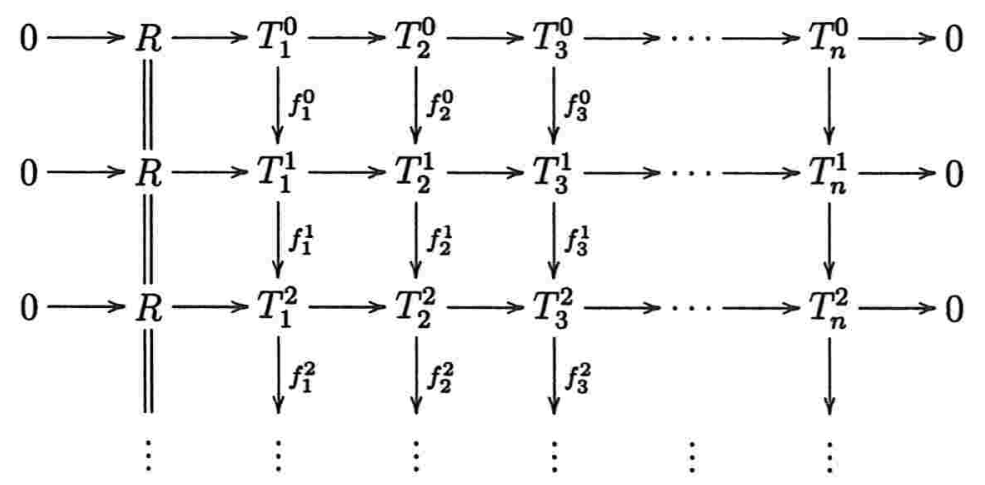

onde alguns $T_{j}^{i}$ podem ser nulos e para cada $i \geq 0$, o par $\left(T_{j}^{i+1}, f_{j}^{i}\right)$, com $f_{j}^{i}$ não nula, é uma $\left(T^{i+1}\right)^{\perp}$-pré-envolvente de $T_{j}^{i}$.

Definamos os morfismos $\phi_{j}^{i, s}=f_{j}^{s} \ldots \circ f_{j}^{i+1} \circ f_{j}^{i}$ se $i \leq s$ e $\phi_{j}^{i, i}=\mathrm{id}_{T_{j}^{i}}$ se $i=s$.

Com os morfismos definidos acima se tomarmos $\left\{T_{j}^{i}, \phi_{j}^{i, s}\right\}$ teremos, para cada $j$ fixado, um sistema direto de módulos com índices em $\mathbb{N}$.

Consideremos para cada $j=1,2, \ldots n$ o limite direto $T_{j}=\lim _{i \in \mathbb{N}}\left(T_{j}^{i}\right)$. Como $\mathbb{N}$ é um conjunto dirigido, então o limite direto é um funtor exato, e portanto teremos a seqüência exata

$$
0 \longrightarrow R \longrightarrow T_{1} \longrightarrow T_{2} \longrightarrow \cdots \longrightarrow T_{n} \longrightarrow 0
$$

Também podemos construir o sistema direto $\left\{\bar{T}^{i}, \phi^{i, s}\right\}_{i \in \mathbb{N}}$, onde cada $\phi^{i, s}=\coprod_{j=1}^{n} \phi_{j}^{i, s}$ e $\bar{T}^{i}=\coprod_{j=1}^{n} T_{j}^{i}$. Seja $T_{j}=\lim _{i \in \mathbb{N}} T_{j}^{i}$ e $T=\coprod_{j=1}^{n} T_{j}$, então $T=\coprod_{j=1}^{n}\left(\lim _{i \in \mathbb{N}}\left(T_{j}^{i}\right) \cong \lim _{i \in \mathbb{N}}\left(\coprod_{j=1}^{n} T_{j}^{i}\right)\right.$. Em outras palavras: se $\bar{T}^{i}=\coprod_{j=1}^{n}\left(T_{j}^{i}\right)$, então $T=\lim _{i \in \mathbb{N}}\left(\bar{T}^{i}\right)$.

Como $\left(T^{i}\right)^{\perp} \subseteq\left(T^{l}\right)^{\perp}$ se $i \geq l$, então $\operatorname{Ext}^{m}\left(\bar{T}^{l}, \bar{T}^{i}\right)=0$ para todo $m>0$ e $i \geq l$. Passaremos agora a construção do sistema inverso de módulos cotiltings ([19]).

Seja $\left\{C_{i}\right\}_{i \in \mathbb{N}}$ uma seqüência de módulos cotiltings finitamente gerados sobre uma álgebra de $\operatorname{Artin} \Lambda \operatorname{com} \operatorname{add} C_{i} \neq \operatorname{add} C_{j}$ se $i \neq j$. Assumiremos que $C_{i} \in{ }^{\perp} C_{i-1}$ para 
todo $i \in \mathbb{N}$. Observe que se a conjectura finitística for válida a última condição não será mais necessária ([27]). Através do Corolário 2.3.6 podemos eliminar a hipótese de $C_{i+1}$ gerar $C_{i}$, então pelo Lema 2.3.5 e pela observação feita no final da seção 2.3 é possível construir por indução um diagrama dual ao diagrama 3.1

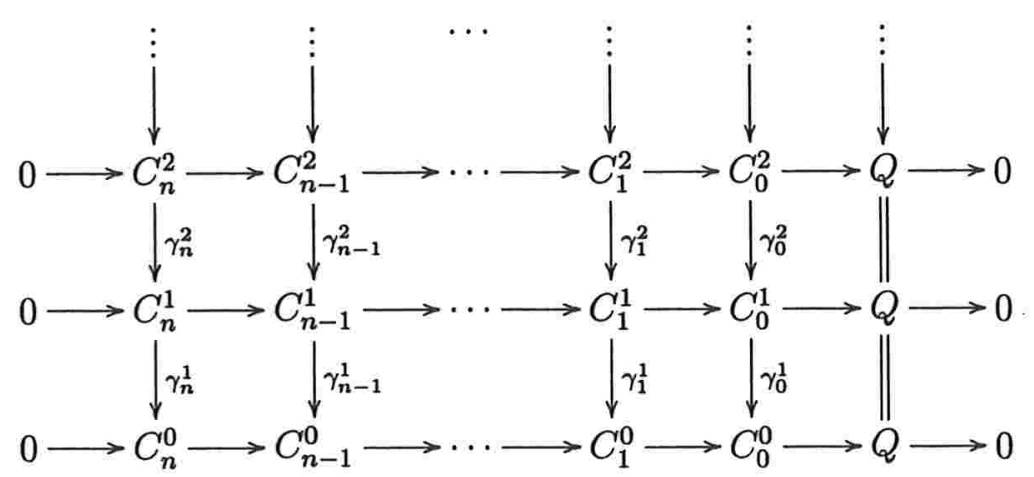

Onde alguns $C_{j}^{i}$ podem ser nulos e $Q$ é um cogerador injetivo finitamente gerado, geralmente usa-se $Q=D \Lambda^{o p}$. Seja $C_{i}^{\prime}=\coprod_{j=0}^{n} C_{j}^{i}$ e $\gamma_{i}$ o morfismo induzido entre $C_{i}^{\prime}$ e $C_{i-1}^{\prime}$ pelos morfismos $\gamma_{j}^{i}$,s.

Então

$$
\cdots \longrightarrow C_{2}^{\prime} \stackrel{\gamma_{2}}{\longrightarrow} C_{1}^{\prime} \stackrel{\gamma_{0}}{\longrightarrow} C_{0}^{\prime}
$$

é um sistema inverso, e $\left(C_{i}^{\prime}, \gamma_{i}\right)$ é uma $C_{i}$-pré-cobertura de $C_{i-1}$.

\subsection{Limites diretos tiltings e limites inversos co- tiltings}

Vamos mostrar agora que $\lim _{i \in \mathbb{N}} \bar{T}_{i}$, como construído na última seção é um módulo $(n+1)$-tilting infinitamente gerado.

Teorema 3.2.1 Sejam $R$ um anel e $\left\{T^{0}, T^{1}, \ldots,\right\}$ uma seqüência de módulos tiltings de tipo finito tais que $\operatorname{Add}^{i} \neq \operatorname{Add}^{j}$ se $i \neq j, T^{i+1} \in\left(T^{i}\right)^{\perp}$ e $\operatorname{pd} T^{i} \leq n$. Então existe uma outra familia $\left\{\bar{T}^{i}\right\}_{i \in \mathbb{N}}$ com $\operatorname{Add} \bar{T}^{i}=\operatorname{Add} T^{i}, \bar{T}^{i+1} \in\left(\bar{T}^{i}\right)^{\perp} e$ 
$\operatorname{pd} \bar{T}^{i} \leq n$ formando um sistema direto de monomorfismos com $T=\lim _{i \in \mathbb{N}} \bar{T}^{i} u m$ módulo tilting em $M o d R$ com $\operatorname{pd} T \leq n+1$.

Prova:

Considere a seqüência $\left\{\bar{T}^{i}\right\}_{i \in \mathbb{N}}$ obtida a partir da seqüência original $\left\{T^{i}\right\}_{i \in \mathbb{N}}$ somando os módulos de cada linha do Diagrama 3.1. Então, como vimos anteriormente, $T=\lim _{i \in \mathbb{N}}\left(\bar{T}^{i}\right)$ e os limites $T_{j}=\lim _{i \in \mathbb{N}} T_{j}^{i}$ das colunas do Diagrama 3.1 resultam na seqüência exata

$$
0 \longrightarrow R \longrightarrow T_{1} \longrightarrow T_{2} \longrightarrow \cdots \longrightarrow T_{n} \longrightarrow 0 .
$$

Então o $R$-módulo regular $R$ admite um corresolução em AddT. Observe que essa seqüência é de fato exata, pois o funtor limite direto é exato ([36]). Portanto $T$ satisfaz a condição $T_{3}$ ) da definição de módulo tilting.

Para verificar que $\operatorname{Ext}^{m}\left(T, T^{(I)}\right)=0$ é suficiente verificar que $T$ se enquadra nas hipóteses do Lema 1.5.7 e do Corolário 1.5.9.

Sabemos que cada $\bar{T}^{i}$ é um $R$-módulo tilting e que $\operatorname{Add}\left(\bar{T}^{i}\right) \subseteq \operatorname{Add}\left(T^{i}\right)$, então $\operatorname{Add}\left(\bar{T}^{i}\right)=\operatorname{Add}\left(T^{i}\right)$ pelo Lema 2.2.1 e, pelo Lema 2.2.3, $\left(T^{i}\right)^{\perp}=\left(\bar{T}^{i}\right)^{\perp}$.

Seja $l \in \mathbb{N}$, como $\left(T^{l}\right)^{\perp}$ é de tipo finito, então também $\left(\bar{T}^{l}\right)^{\perp}$ é de tipo finito. Portanto existe uma classe $\mathcal{S}_{l} \subseteq \mathrm{FP}_{\infty}(R)$ tal que $\left(\bar{T}^{l}\right)^{\perp}=\mathcal{S}_{l}^{\perp}$ pela Proposição 2.1.6. Então para cada $X \in \mathcal{S}_{l}$ temos,

$$
\operatorname{Ext}^{m}(X, T)=\operatorname{Ext}^{m}\left(X, \lim _{i \in \mathbb{N}} \bar{T}^{i}\right) \cong \lim _{i \in \mathbb{N}} \operatorname{Ext}^{m}\left(X, \bar{T}^{i}\right)=0
$$

para todo $m>0$ pelo Lema 1.5.10, pois $\operatorname{Ext}^{m}\left(X, \bar{T}^{i}\right)=0$ para todo $i \geq l$. Disso segue que $T \in\left(\bar{T}^{l}\right)^{\perp}$ para todo $l \in \mathbb{N}$ e como $\left(T^{l}\right)^{\perp}$ é fechada para coprodutos $T^{(I)} \in\left(T^{l}\right)^{\perp}$ para qualquer conjunto de índices $I$. Pela definição do sistema direto de módulos do Diagrama 3.1 acima, para cada $k$ fixado $\left(T_{k}^{l+1}, f_{k}^{l}\right)$ é uma $\left(T^{l+1}\right)^{\perp}$ pré-envolvente injetora de $T_{k}^{l}$.

Seja $C_{k}^{l+1}$ o conúcleo de $f_{k}^{l}$ para $l \geq 0 C_{k}^{0}=T_{k}^{0}$. Com isto, temos para cada $k \in\{1,2, \ldots, n\}$ uma seqüência exata

$$
0 \longrightarrow T_{k}^{l} \stackrel{f_{k}^{l}}{\longrightarrow} T_{k}^{l+1} \longrightarrow C_{k}^{l+1} \longrightarrow 0 .
$$


Somando essas seqüências obtemos

$$
0 \longrightarrow \bar{T}^{l} \stackrel{f^{l}}{\longrightarrow} \bar{T}^{l+1} \longrightarrow \coprod_{k=1}^{n} C_{k}^{l+1} \longrightarrow 0 .
$$

Como $T^{(I)} \in\left(T^{l}\right)^{\perp}$ para todo $l \leq 0$. Aplicando $\operatorname{Hom}\left(-, T^{(I)}\right)$ à seqüência acima tem-se

$\operatorname{Hom}\left(\bar{T}^{l+1}, T^{(I)}\right) \stackrel{\left(f^{l}\right)^{*}}{\longrightarrow} \operatorname{Hom}\left(\bar{T}^{l}, T^{(I)}\right) \longrightarrow \operatorname{Ext}^{1}\left(\coprod_{k=1}^{n} C_{k}^{l+1}, T^{(I)}\right) \longrightarrow \operatorname{Ext}^{1}\left(\bar{T}^{l+1}, T^{(I)}\right)$ $\operatorname{com}\left(f^{l}\right)^{*}$ sobrejetora, então $\operatorname{Ext}^{1}\left(\coprod_{k=1}^{n} C_{k}^{l+1}, T^{(I)}\right)=0$. Como $\left(\bar{T}^{l+1}\right)^{\perp} \subseteq\left(\bar{T}^{l}\right)^{\perp}$ segue que $\operatorname{Ext}^{m}\left(\bar{T}^{l}, T^{(I)}\right) \cong \operatorname{Ext}^{m+1}\left(\bar{T}^{l+1}, T^{(I)}\right)=0$ para todo $m \geq 2$. Então

$$
\operatorname{Ext}^{m}\left(\coprod_{k=1}^{n} C_{k}^{l+1}, T^{(I)}\right)=0
$$

para $m \geq 2$. Portanto

$$
\operatorname{Ext}^{m}\left(\coprod_{k=1}^{n} C_{k}^{l+1}, T^{(I)}\right)=0 \quad \text { para todo } m>0 .
$$

Seja $A_{0}=0, A_{l}=\bar{T}^{l-1}$ e $g_{l, l+1}: A_{l} \rightarrow A_{l+1}$, onde $g_{0,1} \cong 0$ e para todo $l>0$ $g_{l, l+1}=f_{l-1}$. Observe que $\frac{A_{1}}{g_{0,1}\left(A_{0}\right)}=T_{0}$ e $\frac{A_{l+1}}{g_{l, l+1}\left(A_{l}\right)}=\frac{\bar{T}^{l}}{f_{l-1}\left(\bar{T}^{l-1}\right)}=\coprod_{k=1}^{n} C_{k}^{i}$. Assim construímos um sistema direto contínuo nas hipóteses do Lema 1.5.7 e do Corolário 1.5.9. Portanto $\operatorname{Ext}^{n}\left(\lim _{i \in \mathbb{N}} A_{i}, T^{(I)}\right)=0$, mas $\lim _{i \in \mathbb{N}} A_{i}=\lim _{i \in \mathbb{N}} \bar{T}_{i}=T$ e assim

$$
\operatorname{Ext}^{m}\left(\lim _{i \in \mathbb{N}} \bar{T}^{i}, T^{(I)}\right)=0
$$

isto é $\operatorname{Ext}^{m}\left(T \cdot T^{(I)}\right)=0$.

Vejamos agora que $T$ tem dimensão projetiva limitada por $n+1$.

Seja $A \in \operatorname{Mod} R$ um módulo qualquer. Sabemos que $\bar{T}^{i} \in \in^{\perp}\left(\left(T^{i}\right)^{\perp}\right)$, portanto $\operatorname{pd}\left(\bar{T}^{i}\right) \leq n$, de onde $\operatorname{Ext}^{m}\left(\bar{T}^{i}, A\right)=0$ para todo $m \geq n+1$ e para todo $i \in \mathbb{N}$. 
Pela seqüência exata

$$
0 \longrightarrow \bar{T}^{l} \stackrel{f^{l}}{\longrightarrow} \bar{T}^{l+1} \longrightarrow \coprod_{k=1}^{n} C_{k}^{l+1} \longrightarrow 0
$$

$\operatorname{Ext}^{m}\left(\coprod_{k=1}^{n} C_{k}^{0}, A\right)=0$ e $\operatorname{Ext}^{m}\left(\coprod_{k=1}^{n} C_{k}^{l+1}, A\right)=0$ para todo $m>n+1$, portanto $\operatorname{pd}\left(\coprod_{k=1}^{n} C_{k}^{l+1}\right) \leq n+1$. Pelo Corolário 1.5.9, $\operatorname{Ext}^{m}(T, A)=0$ para todo $m>n+1$ e portanto $\operatorname{pd}(T) \leq n+1$.

Poderiamos tentar repetir aqui o argumento dual ao utilizado acima para tentar obter módulos cotiltings infinitos como limites inversos de seqüências de módulos cotiltings como a do Diagrama 3.2. Entretanto o método usado acima não pode ser dualizado, pois embora tenhamos o Lema 3.2.2 abaixo, nem sempre é possível comutar os funtores $\mathrm{Ext}^{m}(-, X)$ com limites inversos, ou seja, em geral

$$
\operatorname{Ext}^{m}\left(\lim _{\leftarrow} M_{i}, N\right) \not \lim _{\leftarrow} \operatorname{Ext}_{m}\left(M_{i}, N\right) .
$$

Lema 3.2.2 ([25] Proposição 18) Seja $\left(A_{\alpha} \mid \alpha \leq \mu\right)$ uma seqüência de módulos, $e$ seja $\left(g_{\beta \alpha} \mid \alpha \leq \beta \leq \mu\right)$ uma seqüência de epimorfismo tais que $\left\{\left(A_{\alpha}, g_{\beta \alpha}\right) \mid \alpha \leq \beta \leq \mu\right\}$ é um sistema inverso contínuo, isto é, $A_{0}=0$ e $A_{\alpha}=\lim _{\dot{\gamma}<\alpha} A_{\gamma}$ para todo ordinal limite $\alpha \leq \mu$. e seja $K_{\alpha}-=\operatorname{Ker}\left(g_{\alpha+1, \alpha}\right)$.

Suponha $C \in \operatorname{Mod} \Lambda$ tal que tal que $\operatorname{Ext}^{1}\left(C, K_{\alpha}\right)=0$ para todo $\alpha+1 \leq \mu$. Então $\operatorname{Ext}^{1}\left(C, A_{\mu}\right)=0$.

Devemos tomar cuidado também ao tentar aplicar a construção de módulos cotiltings de Buan e Solberg ([19]) no caso geral, pois o argumento da demonstração apóia-se no funtor de dualidade $D$ que não é reflexivo em geral. Veja a demonstração de 3.2.6.

Proposição 3.2.3 Considere $T=\lim _{i \in \mathbb{N}} \bar{T}^{i}$ obtido no Teorema 3.2.1. Então $T$ é um módulo tilting de tipo finito e $T=\lim _{\overline{\sigma \in}} C_{\sigma}$ com cada $C_{\sigma} \in \mathrm{FP}_{\infty}(R)$. 
Prova: Seja $T=\lim _{i \in \mathbb{N}}\left(\bar{T}^{i}\right)$. Como $T \in\left(\bar{T}^{i}\right)^{\perp}$ para todo $i \in \mathbb{N}$, então $T \in \bigcap_{i \in \mathbb{N}}\left(\bar{T}^{i}\right)^{\perp}$. Sendo que cada $\bar{T}^{i}$ é um $R$-módulo tilting, $T^{\perp} \subset \bigcap_{i \in \mathbb{N}}\left(\bar{T}^{i}\right)^{\perp}=\bigcap_{i \in \mathbb{N}}\left(T^{i}\right)^{\perp}$ pelos Lemas 2.2.1 e 2.2.3. Isso prova a primeira inclusão. Para a outra inclusão, seja $X \in$ $\bigcap_{i \in \mathbb{N}}\left(\bar{T}^{i}\right)^{\perp}$. Como $\operatorname{Ext}^{m}\left(\coprod_{k=1}^{n} C_{k}^{l}, X\right)=0$, para todo $l \geq 0$, então

$$
\operatorname{Ext}^{m}\left(\lim _{i \in \mathbb{N}} \bar{T}^{i}, X\right)=0
$$

pelos Lemas 1.5.7 e 1.5.9. Portanto $X \in T^{\perp}$. Logo

$$
T^{\perp}=\bigcap_{i \in \mathbb{N}}\left(\bar{T}^{i}\right)^{\perp} .
$$

Observe que $\left(\bar{T}^{i}\right)^{\perp}=S_{i}^{\perp}$ para $S_{i} \subset \mathrm{FP}_{\infty}(R)$, então $\bigcap_{i \in \mathbb{N}}\left(\bar{T}^{i}\right)^{\perp}=\bigcap_{i \in \mathbb{N}} S_{i}^{\perp}=\left(\bigcup_{i \in \mathbb{N}} S_{i}\right)^{\perp}$. Então $T$ é um $R$-módulo tilting de tipo finito.

Um fato importante é que, sendo $T$ de tipo finito, é que se fizermos $\mathcal{X}=T^{\perp}$, então $T=\lim _{\sigma \in I} C_{\sigma}$ com cada $C_{\sigma} \in \mathcal{X} \cap \mathrm{FP}_{\infty}(R)$ por [8] 2.61.

Apresentaremos a seguir, para completude do texto, a versão de Buan e Solberg ([18] ou [19]) do teorema de construção de módulos cotiltings a partir de um limite inverso de uma seqüência cotilting de módulos.

Lema 3.2.4 Sejam $\Lambda$ uma álgebra de Artin e

$$
\cdots \longrightarrow M_{2} \longrightarrow M_{1} \longrightarrow M_{0}
$$

um sistema inverso de módulos em $\bmod \Lambda$. Então $\underset{i \in \mathbb{N}}{\lim } M_{i}$ é um módulo injetivo puro e portanto um somando direto de $\prod_{i \in \mathbb{N}} M_{i}$.

Prova: Considere o sistema direto dual

$$
D M_{0} \longrightarrow D M_{1} \longrightarrow M_{2} \longrightarrow \cdots
$$


e considere o colimite $\lim _{i \in \mathbb{N}} D M_{i}$. Então $D \lim _{i \in \mathbb{N}} D M_{i} \cong \lim _{i \in \mathbb{N}} D D M_{i} \cong \lim _{i \in \mathbb{N}} M_{i}$ que é injetivo puro, pois o dual que qualquer módulo é injetivo puro [30]. Segue também de [30] que a seqüência exata

$$
0 \longrightarrow \lim _{i \in \mathbb{N}} M_{i} \stackrel{f}{\longrightarrow} \prod_{i \in \mathbb{N}} D M_{i} \longrightarrow \operatorname{Coker}(\mathrm{f}) \longrightarrow 0
$$

cinde. Portanto $\lim _{i \in \mathbb{N}} M_{i}$ é um somando de $\prod_{i \in \mathbb{N}} D M_{i}$.

Lema 3.2.5 Sejam $\Lambda$ uma álgebra de Artin e $N$ um $\Lambda$-módulo $n$-cotilting finitamente gerado, então $\mathcal{X}=\left({ }^{\perp} T\right) \cap \bmod \Lambda$ é uma classe de recobrimento em $\bmod \Lambda$.

Prova: Por [12] $5.5\left({ }^{\perp} T\right) \cap \bmod \Lambda$ é uma subcategoria contravariantemente finita resolvente de $\bmod \Lambda$. Se $C$ é um $\Lambda$-módulo e $(X, f)$ uma $\mathcal{X}$ - aproximação minimal à direita de $C$, como $\mathcal{X}$ contém todos os projetivos, então $f$ é sobrejetora. Considere a seqüência exata

$$
0 \longrightarrow K \longrightarrow X \stackrel{f}{\longrightarrow} C \longrightarrow 0 .
$$

Por [12] 3.5, $K \in \mathcal{X}^{\perp}$. Portanto $(X, f)$ é uma pré-cobertura especial. Logo $\mathcal{X}$ é uma classe de recobrimento.

Teorema 3.2.6 Seja $\left\{C_{i}\right\}_{i \in \mathbb{N}}$ uma familia infinita de módulos cotilting com $\operatorname{id} C_{i} \leq$ $n$ para algum $n>0$, tal que $C_{i} \in^{\perp} C_{i-1}$ para cada $i>0$. Então o limite inverso $X=\lim _{i \in \mathrm{N}} C_{i}^{\prime}$ do correspondente sistema inverso é um módulo cotilting infinitamente gerado $\operatorname{com}$ id $X \leq n$.

\section{Prova:}

Pela construção do Diagrama 3.2 podemos ver que cada coluna é constituída de funções sobrejetoras, portanto tomando-se o limite inverso $X_{i}=\lim _{i \in \mathbf{N}} C_{i}^{j}$ em cada coluna teremos uma seqüência exata

$$
0 \longrightarrow X_{n} \longrightarrow X_{n-1} \longrightarrow \cdots \longrightarrow X_{1} \longrightarrow X_{0} \longrightarrow Q \longrightarrow 0
$$


Veja [24] Proposição 1.5.13. Por outro lado, $\lim _{i \in \mathbb{N}} C^{\prime}=\coprod_{i \in \mathbb{N}} \lim _{i \in \mathbb{N}} C_{j}^{i}$, ou seja $X=\coprod_{i \in \mathbb{N}} X_{i}$. Portanto $\mathrm{X}$ satisfaz a condição $\mathrm{C}_{3}$ da definição de módulo cotilting.

Seja $J$ um conjunto qualquer e considere seqüência exata pura

$$
0 \longrightarrow \lim _{i \in \mathbb{N}} C_{i}^{\prime} \stackrel{f}{\longrightarrow} \prod_{i \in \mathbb{N}} C^{\prime} i \longrightarrow \operatorname{Coker} f \longrightarrow 0
$$

Sendo cada $C_{i}^{\prime}$ finitamente gerado, então $C_{i}^{\prime} \cong D D C_{i}^{\prime}$ e pelo Lema 3.2.4 existe sistema direto $\left\{D C_{i}^{\prime}, D\left(\gamma_{i}\right)\right\}$ com limite direto $T=\lim _{i \in \mathbb{N}} D C_{i}^{\prime}$. Portanto $\lim _{i \in \mathbb{N}} C_{i}^{\prime}=$ $D \lim _{i \in \mathbb{N}} D C_{i}^{\prime}$. Dessa forma $\lim _{i \in \mathbb{N}} C_{i}^{\prime}$ é injetivo puro e a seqüência exata acima cinde. Logo $\lim _{i \in \mathbb{N}} C_{i}^{\prime}$ é um somando de $\prod_{i \in \mathbb{N}} C_{i}^{\prime}$. Como $\operatorname{Ext}^{m}\left(\prod_{i \in \mathbb{N}} C_{i}^{\prime}, C_{j}\right)=0$, então $\operatorname{Ext}^{m}\left(X, C_{j}\right)=0$ e também para qualquer produto direto $X^{J}$ de $X$ temos que $\operatorname{Ext}^{m}\left(X^{J}, C_{j}\right)=0$. Sendo $X$ um somando direto de $\prod_{i \in \mathbb{N}} C_{i}^{\prime}$ e $\operatorname{Ext}^{m}\left(X^{J}, \coprod_{i \in \mathbb{N}} C_{i}\right) \cong \coprod_{i \in \mathbb{N}} \operatorname{Ext}^{m}\left(X^{J}, C_{i}\right)=0$, então $\operatorname{Ext}^{m}\left(X^{J}, X\right)=0$ e, portanto, $X$ satisfaz a condição $\mathrm{C}_{2}$ da definição de módulo cotilting. Novamente usando o fato de que $X$ é um somando direto de $\prod_{i \in \mathbb{N}} C^{\prime} i$ temos que $\operatorname{id} X \leq n$.

Na demonstração acima eliminamos a hipótese $C_{i} \in \operatorname{Gen}\left(C_{i-1}\right)$ do teorema original de [19] devido ao Lema 2.3.6.

Definição 3.2.7 1. Seja T um módulo tilting obtido como no Teorema 3.2.1. Dizemos que $T$ é um módulo tilting colimite da seqüência $\left\{T^{i}\right\}_{i \in \mathbb{N}}$ e vamos chamá-lo de t-colimite.

2. Seja $C$ um módulo cotilting obtido como no Teorema 3.2.6. Dizemos que $C$ é um módulo cotilting limite inverso da seqüência $\left\{C_{i}\right\}_{i \in \mathbb{N}}$ e vamos chamá-lo de ct-limite.

Abaixo ilustraremos um exemplo para mostrar como podemos obter um tilting infinito e também mostrar a existência de tais módulos sobre álgebras hereditárias. 
No capítulo 4 veremos mais detalhes de como conseguir módulos $t$-colimites e ctlimites sobre álgebras inclinadas.

Uma álgebra de artin $\Lambda$ é hereditária se todo submódulo de um $\Lambda$-módulo projetivo é também projetivo ou equivalentemente, se gldim $\Lambda \leq 1$.

Exemplo: Seja $\Lambda$ uma álgebra de Artin hereditária básica e indecomponível de tipo de representação infinita.

Considere a decomposição $T_{0}=\coprod_{i=1}^{n} P_{i}$ de $\Lambda$ em $\Lambda$-módulos projetivos indecomponíveis. Para $j \in \mathbb{N}$ defina $T_{j}=\coprod_{i=1}^{n} \tau^{-j} P_{i}$, onde $\tau$ é o transladado de Auslander Reiten. Então a seqüência $\left\{T_{i}\right\}_{\in \mathbb{N}}$ satisfaz as condições do Teorema 3.2.1.

De fato, como $\Lambda$ hereditária, então gldim $\Lambda \leq 1 . \operatorname{Logo}, \operatorname{pd} T_{i} \leq 1 \quad \forall i \in \mathbb{N}$.

Para cada par $(i, j)$,

$$
\operatorname{Ext}^{1}\left(T_{j}, T_{j+1}\right) \cong \coprod_{i, k} \operatorname{Ext}^{1}\left(\tau^{-j} P_{i}, \tau^{-j-1} P_{k}\right) \cong \coprod_{i, k} \operatorname{DHom}\left(\tau^{-j-1} P_{k}, \tau^{-j+1} P_{i}\right)=0,
$$

pela fórmula de Auslander Reiten 1.6.6 e $\operatorname{Ext}^{1}\left(T_{j}, T_{j}\right)=\coprod_{i, k} \operatorname{Ext}^{1}\left(\tau^{-j} P_{i}, \tau^{-j} P_{k}\right) \cong$ $\coprod_{i, k} \operatorname{DHom}\left(\tau^{-j} P_{k}, \tau^{-j+1} P_{i}\right)=0$ por [13] VIII 1.4. Portanto cada $T_{j}$ é autoortogonal e $T_{j} \in T_{j+1}^{\perp}$. Como cada $T_{j}$ tem o mesmo número de somandos que o número de $\Lambda$-módulos simples ${ }^{1}$, cada $T_{i}$ é um módulo tilting. Além disso add $T_{j+1} \neq \operatorname{add} T_{j}$ se $i \neq j$. Segue do teorema de decomposição de Azumaya ([26] 21.6) que $\operatorname{Add} T_{i} \neq$ $\operatorname{Add} T_{j}$ se $i \neq j$. Portanto existe um módulo $t$-colimite sobre álgebras hereditárias, obtido como limite direto de módulos de tipo finito, mais precisamente de módulos finitamente gerados.

\footnotetext{
${ }^{1}$ Veja, [1] para maiores detalhes e a prova de que número de somandos de um módulos tilting é o número de módulos simples da álgebra, esse fato é uma conseqüência do Lema de Bongartz [16] e da equivalência entre AddT e a subcategoria dos $B$-módulos projetivos.
} 
Veja abaixo dois exemplos de seqüências tiltings em componentes pós-projetivas do quiver de Auslander Reiten de duas álgebras de dimensão finita.

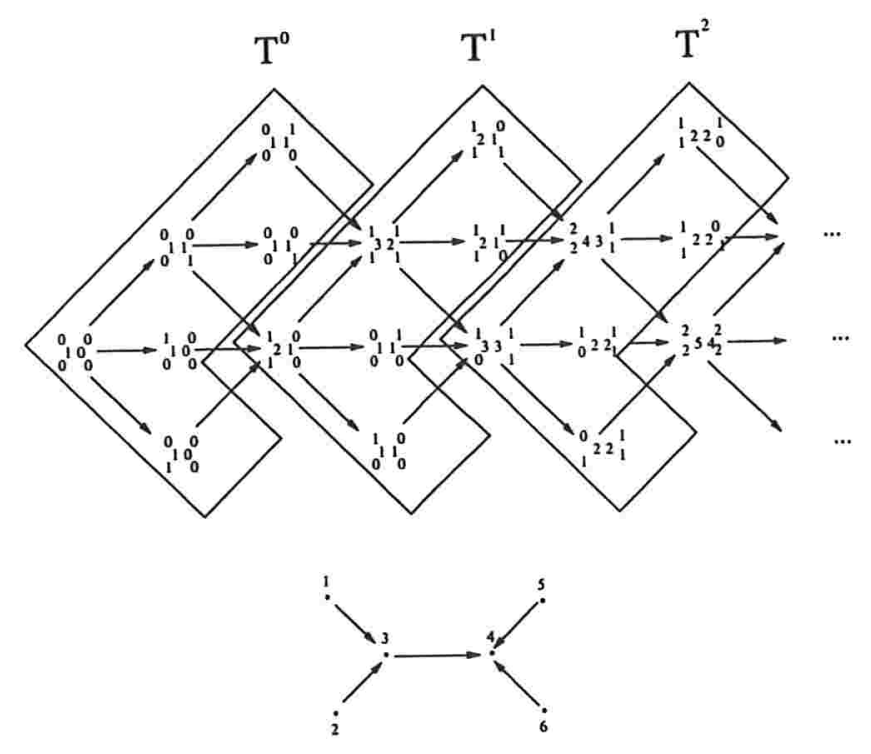

Figura 3.1: Seqüência tilting para uma álgebra hereditária 


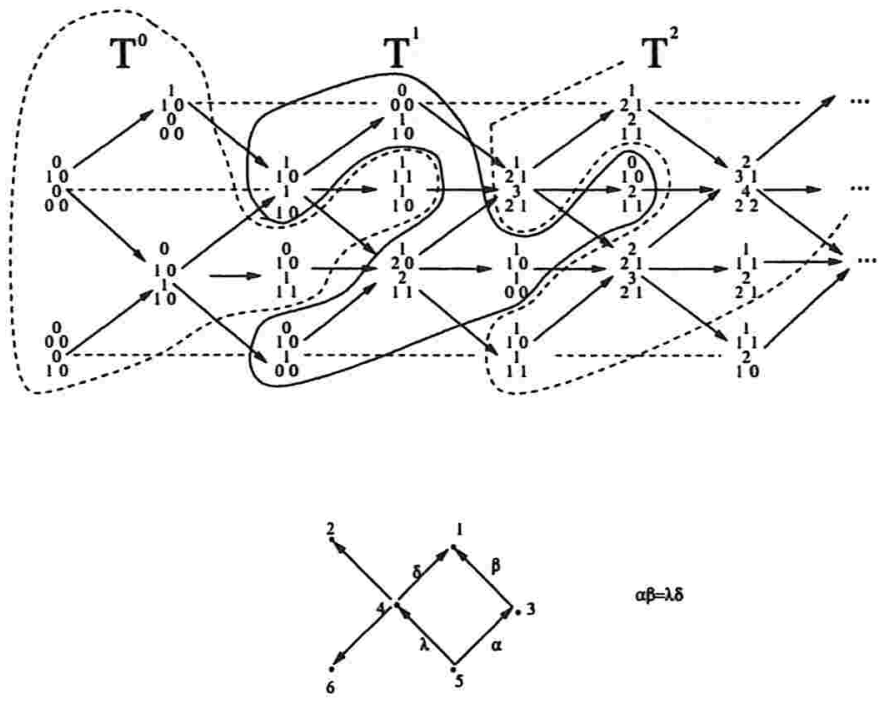

Figura 3.2: Seqüência tilting para uma álgebra inclinada 


\section{Capítulo 4}

\section{Álgebras com módulos tiltings infinitos}

Após termos estabelecido no Capítulo 3 um processo de construção de módulos tiltings não finitamente gerados e estudado a construção de módulos cotiltings não finitamente gerados, apresentaremos nesse capítulo algumas classes de álgebras onde é possível construir esses tipos de módulos. Para o caso cotilting usaremos o resultado de [19] para construir seqüências de módulos cotiltings na categoria de módulos de uma álgebra de dimensão finita, cujo limite inverso ainda seja um cotilting. Estudaremos os módulos tiltings infinitamente gerados sobre álgebras hereditárias de tipo de representação infinito, álgebras inclinadas (ver definição mais adiante) com componentes de conexão estáveis à direita e álgebras cujos quivers de Auslander Reiten contenham uma componente pós-projetiva standard generalizada estável à direita e contendo uma seção finita, Teorema (4.5.3). Apresentaremos também as versões para cotilting quando for possível. Os módulos que estudaremos aqui são os colimites tiltings e limites inversos cotiltings obtidos no Capítulo 3.

A idéia central é construir sobre uma componentes de conexão de uma $\mathbb{K}$-álgebra inclinada de dimensão finita uma seqüência tilting (cotilting) utilizando os funtores de translação de Auslander Reiten e um módulo tilting (cotilting) $T_{0}$ fixado. Esse módulo inicial será a soma direta dos módulos sobre um slice nessa componente de 
conexão. Além disso, vamos verificar que sobre componentes de conexão estáveis à direita, qualquer seqüência tilting crescente gera um sistema direto cujo limite direto é um módulo tilting. Também veremos que sobre essas componentes as seqüências tiltings geram limites diretos Add-equivalente e, portanto com os mesmos somando diretos indecomponíveis. Esse último fato, permite concluir também que a componente está associada a um único tilting maximal.

Em geral nesse capítulo estaremos trabalhando com categorias de módulos à esquerda, entretanto em alguns momentos necessitaremos de módulo à direita, quando for o caso, indicaremos por $X_{A}$ para dizer que $X$ é um $A$-módulo à direita.

\section{1 Álgebras inclinadas}

Nessa seção trabalharemos com uma classe de álgebras um pouco mais geral que álgebras hereditárias, as inclinadas (ou tilted em inglês) que são álgebras que possuem uma estrutura muito próxima das hereditárias e características muito semelhantes e a estrutura do quiver de Auslander Reiten dessas álgebras são bem conhecidas ([1],[29],[34],[35]).

Para o estudo desse capítulo vamos admitir que todas as álgebras utilizadas sejam de dimensão finita sobre um corpo $\mathbb{K}$.

Definição 4.1.1 Seja A uma álgebra. Dizemos que um A-módulo finitamente gerado $T$ é um tilting clássico se $T$ for 1-tilting.

Dizemos que uma álgebra $B$ é inclinada se existe uma $\mathbb{K}$-álgebra hereditária $A$ e um $A$-módulo tilting clássico $T$ tal que $B=\operatorname{End}_{A} T$.

Vamos supor que o módulo ${ }_{A} T$ seja livre de multiplicidade. ${ }^{1}$ Nesse caso $B$ será sempre uma álgebra básica e conexa.

Observação: Qualquer álgebra hereditária é inclinada: de fato, seja $T={ }_{A \text { op }} A$, então $A \cong \operatorname{End}_{A^{\text {op }}} A$.

\footnotetext{
${ }^{1}$ Livre de multipliciada significa que o módulo não possui somandos indecomponíveis repetidos quando escrito como soma direta de indecomponíveis.
} 
Um caminho de $X$ para $Y$ em $\bmod B$ é uma sucessão de morfismos não nulos

$$
X \longrightarrow X_{1} \longrightarrow X_{2} \longrightarrow \ldots X_{n}=Y
$$

Sejam $B$ uma álgebra inclinada e $\mathcal{C}$ uma subcategoria plena de $\bmod B$ fechada para isomorfismos e somandos diretos. A subcategoria $\mathcal{C}$ é fechada para predecessores se: sempre que $L \rightarrow \ldots \rightarrow M$ for um caminho em $\bmod B$, com $M \in \mathcal{C}$ então $L \in \mathcal{C}$. De forma análoga $\mathcal{C}$ é fechada para sucessores se: sempre que $L \rightarrow \ldots \rightarrow M$ for um caminho em $\bmod B \operatorname{com} L \in \mathcal{C}$, então $M \in \mathcal{C}$.

Definição 4.1.2 Seja A uma $\mathbb{K}$-álgebra. Um par de torção em $\bmod A$, é um par de subcategorias $(\mathcal{X}, \mathcal{Y})$ tais que:

a) $\operatorname{Hom}(X, Y)=0$ para todo $X \in \mathcal{X}$ eY $\in \mathcal{Y}$;

b) $\operatorname{Hom}\left(X,{ }_{-}\right)_{\mid} y=0$ implica que $X \in \mathcal{X}$;

c) $\operatorname{Hom}(-, Y)_{\mid \mathcal{X}}=0$ implica que $Y \in \mathcal{Y}$.

Observe que a definição de par de cotorção no Capítulo 1 e a definição acima são definições similares, apenas troca-se o funtor $\operatorname{Hom}(-,,-)$ pelo funtor $\operatorname{Ext}^{1}\left({ }_{-},-\right)$. A idéia da construção de pares de torção é bastante antiga e tem sua origem na teoria de grupos com as classes dos grupos de torção e grupos livres de torção. Trabalharemos apenas com classes de torção geradas por um módulo tilting clássico $T$, conforme definiremos abaixo.

Dada uma álgebra inclinada $B=\operatorname{End}_{A} T$, o módulo $T$ define pares de torção em $\bmod A$ e em $\bmod B$.

O par de torção definido por ${ }_{A} T$ em $\bmod A$ é o par $(\mathcal{F}, \mathcal{T})$, onde

$$
\mathcal{T}=\operatorname{Ker}\left(\operatorname{Ext}^{1}\left(T,{ }_{-}\right)\right)
$$

e

$$
\mathcal{F}=\left\{N \in \bmod A \text { tais que } \operatorname{Hom}_{\mid T}(-, N)=0\right\} .
$$

O par de torção definido por ${ }_{A} T$ e $\bmod B$ é $(\mathcal{Y}(T), \mathcal{X}(T))$, onde

$$
\mathcal{Y}(T)=\{Y \in \bmod B / \text { existe } X \in \mathcal{T} \operatorname{com} Y=\operatorname{Hom}(T, X)\}=\operatorname{Ker}\left(\operatorname{Tor}_{B}(T,-)\right)
$$




$$
\mathcal{X}(T)=\left\{Y \in \bmod B / \text { existe } X \in \mathcal{F} \operatorname{com} Y=\operatorname{Ext}^{1}(T, X)\right\}=\operatorname{Ker}\left(T_{B} \otimes_{-}\right)
$$

Para os pares de torção acima valem os seguintes fatos:

a) Qualquer seqüência exata está inteiramente em $\mathcal{X}(T)$ ou inteiramente em $\mathcal{Y}(T)$ ou é uma seqüência de conexão, isto é, o termo à esquerda está em $\mathcal{Y}(T)$ e o termo à direita em $\mathcal{X}(T)$. A componente do quiver de Auslander Reiten que contém as seqüências de conexão será chamada de componente de conexão. Uma álgebra inclinada pode ter uma ou duas componentes de conexão e terá duas se e somente se a álgebra for concealed, ou seja $B=\operatorname{End}_{A} T$ onde todos os somandos do $A$-módulo tilting $T$ são pós-projetivos (ou pré-injetivos).

b) Qualquer $B$-módulo indecomponível tem dimensão projetiva menor ou igual a 1 ou dimensão injetiva menor ou igual 1 e gldim $B \leq 2$.

Os pares de torção em $\bmod A$ e em $\bmod B$ estão associados pelo teorema de Brenner-Butler [17] como veremos abaixo.

Para que não haja confusão indicaremos sobre qual álgebra os funtores estão agindo.

Teorema 4.1.3 (Teorema de Brenner-Butler) Sejam A uma álgebra hereditária, $T$ um A-módulo tilting, $B=\operatorname{End} T,(\mathcal{T}(T), \mathcal{F}(T)),(\mathcal{X}(T), \mathcal{Y}(T))$ os pares de torção induzidos por $T \bmod A$ e $\bmod B$ respectivamente. Então

(a) $T_{B}$ é um módulo tilting, e o morfismo canônico $A \longrightarrow \mathrm{End}_{B}$ definido por $a \longmapsto(t \longmapsto a t)$ é um isomorfismo.

b) Os funtores $\operatorname{Hom}_{A}\left(T,{ }_{-}\right)$e $T_{B} \otimes_{-}$induzem equivalências quase inversas entre $\mathcal{T}(T)$ e $\mathcal{Y}(T))$

b) Os funtores $\operatorname{Ext}_{A}^{1}(T,-)$ e $\operatorname{Tor}_{1}^{B}\left(T_{B},-\right)$ induzem equivalências quase inversas entre $\mathcal{F}(T)$ e $\mathcal{X}(T))$

Encorajamos o leitor a consultar [1] ou [17] para a prova do teorema. 
As equivalências parciais dadas pelo Teorema de Brenner Butler permite transferir informações entre a álgebra hereditária $A$ e a álgebra inclinada $B$ e conhecer melhor a estrutura da categoria de módulos da álgebra inclinada $B$.

Definição 4.1.4 Seja $B$ uma $\mathbb{K}$-álgebra. Um par de torção $(\mathcal{X}, \mathcal{Y}) \bmod B$ é splitting se qualquer módulo indecomponivel está em uma das categorias $\mathcal{X}$ ou $\mathcal{Y}$.

Lema 4.1.5 Sejam A uma álgebra hereditária, $T$ um A-módulo tilting clássico e $B=$ End $T$. Então:

a) $O$ par de torção $(\mathcal{X}(T), \mathcal{Y}(T))$ em $\bmod B$ é splitting.

b) $\mathcal{Y}(T)$ é fechado para predecessores e $\mathcal{X}(T)$ é fechado para sucessores.

Prova: Veja [1] ou [34] para a demonstração.

\subsection{Tilting e cotiltings infinitos em álgebras incli- nadas}

Nessa seção veremos que sobre algumas álgebras inclinadas é possível obter módulos tiltings e cotiltings infinitos pelos métodos estudados no Capítulo 3 .

Iniciaremos a seção com as definições e os lemas que nos ajudarão no decorrer do capítulo.

Seja $Q=\left(Q_{0}, Q_{1}, s, t\right)$ um quiver finito, conexo e acíclico e seja $n=\left|Q_{0}\right|$. Para todo vértice $a \in Q_{0}$ definiremos um novo quiver $\sigma_{a} Q=\left(Q_{0}^{\prime}, Q_{1}^{\prime}, s^{\prime}, t^{\prime}\right)$ da seguinte forma: Todas as flechas que tem $a$ como vértice inicial ou final são revertidas e as demais permanecem inalteradas. Em outras palavras $Q_{0}^{\prime}=Q_{0}$ e existe uma bijeção $Q_{1} \rightarrow Q_{1}^{\prime}$ definida por $\alpha \mapsto \alpha^{\prime}$ tal que, se $\alpha \in Q_{1}$ então

(i) se $s(\alpha) \neq a$ e $t(\alpha) \neq a$, então $s^{\prime}\left(\alpha^{\prime}\right)=s(\alpha)$ e $t^{\prime}\left(\alpha^{\prime}\right)=t(\alpha)$; 
(ii) se $s(\alpha)=a$ ou $t(\alpha)=a$, então $s^{\prime}\left(\alpha^{\prime}\right)=t(\alpha)$ e $t^{\prime}\left(\alpha^{\prime}\right)=s(\alpha)$.

Seja $Q$ um quiver conexo e acíclico. Uma seqüência admissível de fontes em $Q$ é uma ordem total $\left(a_{1}, \ldots, a_{n}\right)$ dos vértices de $Q$ tal que:

(i) $a_{1}$ é uma fonte em $Q$

(ii) $a_{i}$ é uma fonte em $\sigma_{a_{i-1}} \ldots \sigma_{a_{1}} Q$ para todo $1 \leq i \leq n$

Dizemos que o quiver $\sigma_{a} Q$ foi obtido de $Q$ por uma reflexão e que $\sigma_{a_{i-1}} \ldots \sigma_{a_{1}} Q$ foi obtido de $Q$ por uma seqüência admissível de reflexões.

Lema 4.2.1 Sejam A uma álgebra de dimensão finita, $X$ e $Y$ A-módulos indecomponíveis e $f$ um morfismo não nulo em $\operatorname{rad}^{\infty}(X, Y)$. Então para cada $t>0$.

(a) Existe um caminho entre A-módulos indecomponíveis

$$
X=X_{0} \stackrel{f_{1}}{\rightarrow} X_{1} \stackrel{f_{2}}{\rightarrow} X_{2} \stackrel{f_{2}}{\rightarrow} \ldots \stackrel{f_{t}}{\rightarrow} X_{t} \stackrel{g_{t}}{\rightarrow} Y
$$

onde $f_{1}, f_{2}, \ldots, f_{t}$ são morfismos irredutíveis e $g_{t} \in \operatorname{rad}^{\infty}\left(\mathrm{X}_{\mathrm{t}}, \mathrm{Y}\right)$.

(b) Existe um caminho entre A-módulos indecomponiveis

$$
X \stackrel{g_{t}^{\prime}}{\rightarrow} Y_{t} \stackrel{f_{t}^{\prime}}{\rightarrow} \ldots \stackrel{f_{3}^{\prime}}{\rightarrow} Y_{2} \stackrel{f_{2}^{\prime}}{\rightarrow} Y_{1} \stackrel{f_{1}^{\prime}}{\rightarrow} Y_{0}=Y
$$

onde $f_{1}^{\prime}, f_{2}^{\prime}, \ldots, f_{t}^{\prime}$ são morfismos irredutiveis e $g_{t}^{\prime} \in \operatorname{rad}^{\infty}\left(\mathrm{X}, \mathrm{Y}_{\mathrm{t}}\right)$.

Prova: Para a prova do lema referenciamos o leitor a [31] Lema 1.2.

Lema 4.2.2 Sejam B uma álgebra, $\mathcal{C}$ uma componente de $\Gamma_{B}$ e $\Delta$ um subquiver pleno finito acíclico e conexo de $\mathcal{C}$.

(a) Assuma que, se $U \in \Delta$ e existe um morfismo irredutivel $V \rightarrow U$, então $V \in \Delta$ ou $V \in \tau \Delta$. Então qualquer morfismo $f: Y \rightarrow U$ entre $B$-módulos indecomponíveis com $Y \notin \Delta$ fatora-se através de uma soma direta de módulos em $\tau \Delta$. 
(b) Assuma que, se $U \in \Delta$ e existe um morfismo irredutivel $U \rightarrow V$, então $V \in \Delta$ ou $V \in \tau^{-} \Delta$. Então qualquer morfismo $g: U \rightarrow X$ com $X \notin \Delta$ fatora-se através de uma soma direta de módulos em $\tau^{-} \Delta$.

Prova: Faremos a prova de (a) visto que a prova de (b) é similar. Sendo $\Delta$ um quiver finito e acíclico, provaremos a afirmação por indução em uma seqüencia admissível de fontes em $\Delta$. Assuma primeiro que $U$ seja uma fonte em $\Delta$, e considere o morfismo minimal quase cindido à direita $u: E \rightarrow U$. Então todo somando indecomponível de $E$ pertence a $\tau \Delta$. Como $f$ não é um epimorfismo que cinde, $f$ fatora-se através de $u$ e a prova está completa. Assumimos agora que $U$ não seja uma fonte, e considere o morfismo minimal quase cindido à direita $u: E \rightarrow U$. Então $E=E^{\prime} \amalg E^{\prime \prime}$, onde todos os somandos indecomponíveis de $E^{\prime}$ pertencem a $\tau \Delta$, enquanto os somandos de $E^{\prime \prime}$ pertencem a $\Delta$ e, portanto, precedem $U$ na seqüencia admissível de fontes. Então $f$ fatora-se através de $u=E^{\prime} \amalg E^{\prime \prime} \rightarrow U$, pois o morfismo $Y \rightarrow E^{\prime \prime}$ fatora-se através de $\tau \Delta$ pela hipótese de indução, e assim completamos a prova.

Definição 4.2.3 Sejam C uma componente conexa do quiver de Auslander Reiten de uma $\mathbb{K}$-álgebra $A$, $\tau$ e $\tau^{-}$os funtores de translação de Auslander Reiten.

Dado um A-módulo indecomponível $X$, definimos a $\tau$-órbita de $X$ como sendo o conjunto formado pelos $Y \in \mathcal{C}$ tais que existe um $n \in \mathbb{Z}$ com $\tau^{n} Y=X$.

Definição 4.2.4 Sejam $A$ uma $\mathbb{K}$-álgebra e $\mathcal{C}$ uma componente do quiver de Auslander Reiten de $A$. Uma seção $\Delta$ em $\mathcal{C}$ é um subquiver de $\mathcal{C}$ satisfazendo:

1) $\Delta$ não contém ciclos orientados;

2) $\Delta$ encontra cada $\tau$-órbita exatamente uma vez;

3) $\mathrm{Se} X_{1} \longrightarrow X_{2} \longrightarrow \ldots \longrightarrow X_{n}$ é um caminho em $\mathcal{C} \operatorname{com} X_{1}$ e $X_{n} \in \Delta$, então $X_{i} \in \Delta$ para todo $1 \leq i \leq n$; 
4) Para cada flecha $X \longrightarrow Y$ em $\mathcal{C}$, se $X \in \Delta$, então $Y \in \Delta$ ou $\tau Y \in \Delta$; e se $Y \in \Delta$, então $X \in \Delta$ ou $\tau^{-} X \in \Delta$.

Definição 4.2.5 Sejam A uma $\mathbb{K}$-álgebra e $\mathcal{C}$ uma componente do quiver de Auslander Reiten de A. Uma subseção $\Sigma$ em $\mathcal{C}$ é um subquiver pleno e conexo de uma seção $\Delta$ de $\mathcal{C}$.

Dizemos que um caminho $X_{0} \rightarrow X_{1} \rightarrow \ldots \rightarrow X_{m}$ entre indecomponíveis em $\bmod A$ é um caminho seccional se cada morfismos for irredutível e $\tau X_{i+1} \neq X_{i-1}$ para $0<i<m$.

Observe que se tivermos um caminho contido em uma seção $\Delta$ de uma componente do quiver de Auslander Reiten de uma álgebra $A$, então esse caminho necessariamente é seccional, pois uma seção encontra cada $\tau$ órbita uma única vez. Logo qualquer subquiver conexo de $\Delta$ tem essa propriedade.

Definição 4.2.6 Uma classe $\mathcal{S}$ em $\bmod A$ é um slice se satisfaz:

1) $\mathcal{S}$ contém um módulo sincero;

2) $S$ e $X_{1} \longrightarrow X_{2} \longrightarrow \ldots \longrightarrow X_{n}$ é um caminho com $X_{1}$ e $X_{n}$ em $\mathcal{S}$, então $X_{i} \in \mathcal{S}$ para todo $1 \leq i \leq n$;

3) $S e M \in \operatorname{ind} A$, então no máximo um entre $M$ e $\tau M$ está em $\mathcal{S}$;

4) Se $f: M \longrightarrow S$ é um morfismo irredutivel em $\bmod A$, com $M$ e $S$ indecomponiveis e $S \in \mathcal{S}$, então $M$ ou $\tau^{-} M \in \mathcal{S}$.

Observação: Um slice em uma componente de conexão define sobre essa componente uma seção no sentido da Definição 4.2.4. Alguns autores usam a nomenclatura "slice completo" e seção plena para os conceitos definidos acima. Caso não haja confusão, usaremos a palavra slice para indicar a seção formada pela classe $\mathcal{S}$ em uma componente do quiver de Auslander Reiten de uma álgebra $B$. 
A seguir faremos uma analogia à teoria de representações de álgebras hereditárias e definiremos subcategorias na componente de conexão. Estas subcategorias terão comportamento similares às componentes pós-projetivas ou pré-injetivas.

Sejam $B=\operatorname{End}_{A} T$ uma álgebra inclinada de dimensão finita e $\mathcal{C}$ a componente de conexão de $\Gamma_{B}$. Seja $\Sigma$ o slice definido por $T$ na componente de conexão $\mathcal{C}$ do quiver de Auslander Reiten de uma álgebra inclinada $B^{2}$, então para um $B$ módulo indecomponível $X \in \mathcal{C}$ existe apenas um único $n \in \mathbb{Z}$ tal que $\tau^{n}(X) \in \Sigma$. Denotaremos esse número por $\nu(X)$ e chamaremos de índice de translação de $X$.

Definição 4.2.7 Sejam $\mathcal{C}$ uma componente de conexão do quiver de Auslander Reiten da álgebra inclinada $B$ e $\Sigma$ o slice definido por ${ }_{A} T$ em $\bmod B$ definimos:

1) $\Sigma^{+}=\left\{X \in \mathcal{C} / \exists n \in \mathbb{N} \operatorname{com} \tau^{n} X \in \Sigma\right\}$

2) $\Sigma^{-}=\left\{X \in \mathcal{C} / \exists n \in \mathbb{N} \operatorname{com} \tau^{-n} X \in \Sigma\right\}$.

Observe que se $X \in \Sigma^{+}$, então $\nu(X) \geq 0$, se $X \in \Sigma^{-}$, então $\nu(X) \leq 0$ e que se $X \in \Sigma^{+} \cap \Sigma^{-}$, então $X \in \Sigma$.

Definição 4.2.8 Seja $\mathcal{C}$ uma componente do quiver de Auslander Reiten de uma álgebra $B$.

1) Dizemos que uma componente $\mathcal{C}$ é estável à direita se não existem módulos injetivos em $\mathcal{C}$. Equivalentemente, $\tau^{-i} X$ existe para todo $i \geq 0$.

2) Dizemos que uma componente $\mathcal{C}$ é estável à esquerda se não existem módulos projetivos em $\mathcal{C}$, ou que $\tau^{i} X$ existe para todo $i \geq 0$.

Proposição 4.2.9 Sejam $B=\operatorname{End} T$ uma $\mathbb{K}$-álgebra inclinada, $\mathcal{C}$ a componente de conexão de $\Gamma_{B}$ que contém o slice $\Sigma$ definido por $T$. Sejam $X$ e $Y \in \mathcal{C}$ tais que $\operatorname{Hom}_{B}(Y, X) \neq 0$, então $\nu(Y) \leq \nu(X)$.

\footnotetext{
${ }^{2} \mathrm{O}$ slice definido por $T$ em $\bmod B$ é a seção formada pelos módulos da forma $\operatorname{Hom}_{A}(T, I) \operatorname{com}$ cada $I$ sendo um $A$-módulo injetivo indecomponível.
} 
Prova: Suponha por contradição que $\nu(X)<\nu(Y)$.

1) Em primeiro lugar, suponha que $\nu(Y) \leq 0$. Como $\tau^{\nu(Y)} Y \in \Sigma \mathrm{e}$

$$
\operatorname{Hom}_{B}(Y, X) \neq 0,
$$

então $\operatorname{Hom}_{B}\left(\tau^{\nu(Y)} Y, \tau^{\nu(Y)} X\right) \neq 0$. De fato, se $\operatorname{Hom}_{B}(Y, X) \neq 0$, então

$$
\overline{\operatorname{Hom}}_{B}(Y, X) \neq 0,
$$

pois caso contrário, toda $f: Y \rightarrow X$ fatora-se por um injetivo e, nesse caso, existe um morfismo não nulo de $I$ para $X$, para algum injetivo $I$. Mas $\nu(X)<0$ e então $X \in \mathcal{Y}(T)$, portanto $I \in \mathcal{Y}(T)$. Como todos os injetivos de $\mathcal{Y}(T)$ estão em $\Sigma$ e $X$ é um sucessor de $I$ em $\mathcal{Y}(T)$, então $X \in \Sigma$. Portanto $\nu(X)=0$ e isso é uma contradição com a nossa hipótese. Logo $\overline{\operatorname{Hom}}_{B}(Y, X) \neq 0$. Aplicando o funtor translação de Auslander Reiten $\tau^{-}$temos que $\operatorname{Hom}_{B}\left(\tau^{-} Y, \tau^{-} X\right) \neq 0$. Repetindo o argumento concluímos que $\operatorname{Hom}_{B}\left(\tau^{\nu(Y)} Y, \tau^{\nu(Y)} X\right) \neq 0$. Logo $\tau^{\nu(Y)} Y \in \Sigma$, existe um morfismo não nulo $f$ de $\tau^{\nu(Y)} Y$ para $\tau^{\nu(Y)} X$. Se $\left.\tau^{\nu(Y)} X\right) \notin \Sigma, f$ fatora-se por $\tau^{-} \Sigma$ pelo Lema 4.2.2, existe $h$ não nula em $\operatorname{Hom}_{B}(\mathcal{X}(T), \mathcal{Y}(T))$. Contradição! Portanto $\left.\tau^{\nu(Y)} X\right) \in \Sigma$ e dessa forma $\nu(Y)=\nu(X)$ o que é uma contradição com nossa hipótese inicial. Logo $\nu(Y) \leq \nu(X)$.

2) Se $\nu(X) \geq 0$, então $\tau^{\nu(X)} X \in \Sigma$ e $\tau^{\nu(X)} Y \in \mathcal{X}(T)$. Nesse caso

$$
\operatorname{Hom}_{B}\left(\tau^{\nu(X)} Y, \tau^{\nu(X)} X\right)=0 \mathrm{e},
$$

portanto $\operatorname{Hom}_{B}(Y, X)=0$, pelo mesmo argumento usado acima. Mas isso contradiz a hipótese da proposição. Portanto $\nu(Y) \leq \nu(X)$.

A proposição acima é equivalente a: se $\nu(X)<\nu(Y)$, então $\operatorname{Hom}_{B}(Y, X)=0$

Definição 4.2.10 Sejam $B=\operatorname{End} T$ uma $\mathbb{K}$-álgebra inclinada, $\mathcal{C}$ uma componente de conexão de $\Gamma_{B}$ e $\Sigma$ o slice definido por $T$ na componente $\mathcal{C}$. Considere $X=\coprod_{j=1}^{k} X_{j}$ com cada $X_{j} \in \mathcal{C}$, definimos $\nu_{d}\left(\coprod_{j=1}^{k} X_{j}\right)=\min \left\{\nu\left(X_{j}\right)\right\}$ e $\nu^{u}\left(\coprod_{j=1}^{k} X_{j}\right)=\max \left\{\nu\left(X_{j}\right)\right\}$. 
Proposição 4.2.11 Sejam $Y=\coprod_{i=1}^{k} Y_{i}$ e $X=\coprod_{j=1}^{m} X_{j} B$-módulos com todos os somandos indecomponíveis em $\mathcal{C}$. Se $\operatorname{Hom}_{B}(Y, X) \neq 0$, então $\nu_{d}(Y) \leq \nu^{u}(X)$.

Prova: Suponha $\operatorname{Hom}(Y, X) \neq 0$, então existem índices $i_{k}$ e $j_{s}$ tais que

$$
\operatorname{Hom}\left(Y_{j_{s}}, X_{i_{k}}\right) \neq 0
$$

Pelo Lema 4.2.9 $\nu\left(Y_{j_{s}}\right) \leq \nu\left(X_{i_{k}}\right)$, e então $\nu_{d}(Y) \leq \nu\left(Y_{j_{s}}\right) \leq \nu\left(X_{i k}\right) \leq \nu^{u}(X)$.

Equivalentemente $\nu^{u}(X)<\nu_{d}(Y) \Rightarrow \operatorname{Hom}(Y, X)=0$.

Teorema 4.2.12 Sejam $B=$ EndT uma álgebra inclinada e $\mathcal{C}$ uma componente de conexão do quiver de Auslander Reiten de $\bmod B$.

1) Suponha que $\mathcal{C}$ é estável à direita. Então existem módulos t-colimites em $\operatorname{Mod} B$.

2) Suponha que $\mathcal{C}$ é estável à esquerda. Então existem módulos ct-limites em $\operatorname{Mod} B$.

Prova: 1) Provaremos que se a componente de conexão $\mathcal{C}$ for estável à direita, então existe uma seqüência tilting constituída por módulos finitamente gerados em $\mathcal{C}$ e o resultado seguirá pelo Teorema 3.2.1.

Primeiro provaremos que $\operatorname{Hom}(D B, Y)=0$ para todo $Y \in \mathcal{C}$.

Como $\mathcal{C}$ estável à direita, não existem injetivos em $\mathcal{C}$. Considere a seção $\Sigma$ definida em $\mathcal{C}$ por ${ }_{A} T, I$ um $B$-módulo injetivo indecomponível e suponha que $\operatorname{Hom}(I, Y) \neq 0$ para algum $Y \in \mathcal{C}$. Como $(\mathcal{X}(T), \mathcal{Y}(T))$ é um par de torção splitting, temos que $Y \in \mathcal{Y}(T)$ ou $Y \in \mathcal{X}(T)$.

a) Se $Y \in \mathcal{Y}(T)$, então $I \in \mathcal{Y}(T)$, pois $\mathcal{Y}(T)$ é fechada para predecessores. Mas nesse caso $I \in \Sigma$, contrariando a hipótese de estabilidade à direita de $\mathcal{C}$.

b) Se $Y \in \mathcal{X}(T)$, como $I \notin \mathcal{C}$, então $f \in \operatorname{rad}^{\infty}(I, Y)$, e pelo Lema 4.2.1 ítem (b), para todo $t>0$ existe um caminho de morfismos

$$
I \stackrel{g_{t}}{\rightarrow} X_{t} \stackrel{f_{t}}{\rightarrow} X_{t-1} \stackrel{f_{t-1}}{\rightarrow} \ldots \stackrel{f_{1}}{\rightarrow} X_{0}=Y
$$


com $f_{i}$ irredutíveis e $g_{t} \in \operatorname{rad}^{\infty}\left(I, X_{t}\right)$. Logo para $t$ suficientemente grande, temos um $X_{i} \in \Sigma$ para algum $0<i \leq t$ e, portanto $X_{t} \in \mathcal{Y}(T)$. Como $\operatorname{Hom}\left(I, X_{t}\right) \neq 0$ segue que $I \in \mathcal{Y}(T)$, e nesse caso $I \in \Sigma$, o que contradiz novamente a hipótese de estabilidade à direita de $\mathcal{C}$.

Portanto $\operatorname{Hom}(D B, Y)=0$ qualquer que seja $Y \in \mathcal{C}$.

Seja $X \in \mathcal{C}$, pelo argumento acima $\operatorname{Hom}(D B, \tau X)=0$, então segue pela Proposição 1.6.5 que $\operatorname{pd} X \leq 1$.

Mostraremos agora a existência da seqüência tilting.

Considere seqüencia de $B$-módulos $\left\{M_{i}\right\}_{i \in \mathbb{N}}$, onde cada $M_{i}$ e a soma dos módulos em $\tau^{-i} \Sigma$. Então essa seqüência é uma seqüência tilting em $\mathcal{C}$.

De fato,

- $\operatorname{pd} M_{i} \leq 1$, pois cada somando de $M_{i}$ está em $\mathcal{C}$.

- Como $\mathcal{C}$ é estável à direita, cada $\tau$-órbita de $\mathcal{C}$ intercepta $\tau^{-i} \Sigma$ uma única vez, portanto $\left|\tau^{-i} \Sigma_{0}\right|=\mid \tau$-órbitas de $\mathcal{C}|=| n_{B} \mid$, onde $n_{B}$ é o número de $B$ módulos simples. Portanto $\tau^{-i} \Sigma_{0}$ é um módulo tilting (Veja [1]). ${ }^{3}$

- $\operatorname{Ext}^{1}\left(M_{i}, M_{j}\right)=0$ para $i \leq j$. De fato, como $\nu^{u}\left(M_{i}\right)<\nu_{d}\left(M_{j+1}\right)$, então $\operatorname{Hom}\left(M_{j+1}, M_{i}\right)=0$ para todo $i \geq 0$. Pela fórmula de Auslander Reiten 1.6.6 $\operatorname{Ext}^{1}\left(M_{i}, \tau M_{j+1}\right)=0$. Logo $\operatorname{Ext}^{1}\left(M_{i}, M_{j}\right)=0$. Observe que $M_{j+1}=\tau^{-} M_{j}$. Assim cada $M_{i+1} \subset M_{i}^{\perp}$.

Portanto cada $M_{i}$ é um módulo tilting e $\left\{M_{i}\right\}_{i \in \mathbb{N}}$ forma uma seqüência tilting em $\bmod B$.

2) Suponhamos $\mathcal{C}$ estável à esquerda, então $\operatorname{Hom}(X, B)=0$ para todo $X \in \mathcal{C}$. De fato, como $\mathcal{C}$ é estável à esquerda não existem projetivo em $\mathcal{C}$. Suponha por absurdo que existe um projetivo indecomponível $P$ e um $B$-módulo $X \in \mathcal{C}$ tal que

\footnotetext{
${ }^{3}$ Veja, [1] para maiores detalhes e a prova de que número de somandos de um módulos tilting é o número de módulos simples da álgebra, esse fato é uma conseqüência do Lema de Bongartz [16] e da equivalência entre Add $T$ e a subcategoria dos $B$-módulos projetivos.
} 
$\operatorname{Hom}(X, P) \neq 0$. Como $P \notin \mathcal{C}$ existe $f \in \operatorname{rad}^{\infty}(X, P)$. Se $\Sigma$ é a seção definida por $T$ em $\bmod B$, então $P \notin \Sigma$. Por outro lado $X \in \mathcal{Y}(T)$, pois se $X \in \mathcal{X}(T)$ teríamos $\operatorname{Hom}(\mathcal{X}, \mathcal{Y}) \neq 0$, sendo que $\mathcal{Y}(T)$ contém todos os projetivos indecomponíveis. Pelo Lema 4.2.2 a) para todo $t>0$ existe uma seqüência

$$
X \stackrel{f_{0}}{\longrightarrow} M_{1} \stackrel{f_{1}}{\longrightarrow} \cdots \longrightarrow M_{t} \stackrel{g_{t}}{\longrightarrow} P
$$

com $f_{i}$ irredutíveis e $g_{t} \in \operatorname{rad}^{\infty}\left(M_{t}, N\right)$. Logo existe um $t_{0}$ tal que $M_{t_{0}} \in \mathcal{Y}(T)$. Portanto $\operatorname{Hom}(\mathcal{X}(T), \mathcal{Y}(T)) \neq 0$, o que é uma contradição, pois o par $(\mathcal{X}(T), \mathcal{Y}(T))$ é um par de torção em $\bmod B$.

Logo $\operatorname{Hom}(X, P)=0$ para todo indecomponível $P$, donde $\operatorname{Hom}(X, B)=0$ para todo $X \in \mathcal{C}$. Conseqüentemente id $X \leq 1$.

- Seja $\left\{M_{i}\right\}$ o $B$-módulo formado pela soma dos $B$-módulos em $\tau^{i} \Sigma$, então id $X \leq$ 1.

- Como $\mathcal{C}$ é estável à esquerda cada $\tau$-órbita intercepta $\tau \Sigma$ uma única vez. Portanto

$\left|\tau^{i} \Sigma_{0}\right|=\mid \tau$-órbitas de $\mathcal{C}|=| n_{B} \mid$, onde $\left|n_{B}\right|$ é o número de $B$-módulos simples.

- $\operatorname{Ext}^{1}\left(M_{i}, M_{j}\right)=0$ se $j \leq i$.

De fato, sabemos que $\nu^{u}\left(M_{i+1}\right)<\nu_{d}\left(M_{j}\right)$ se $j \leq i$, então

$$
\operatorname{Hom}\left(M_{j}, M_{i+1}\right)=0
$$

pela Proposição 4.2.11. Mas, nesse caso, $\overline{\operatorname{Hom}}\left(M_{j}, M_{i+1}\right)=0$. Como

$$
\overline{\operatorname{Hom}}\left(M_{j}, M_{i+1}\right)=\overline{\operatorname{Hom}}\left(M_{j}, \tau M_{i}\right)
$$

e, $\operatorname{Ext}^{1}\left(M_{i}, M_{j}\right)=0$ se e somente se

$$
\overline{\operatorname{Hom}}\left(M_{j}, \tau M_{i}\right)=0
$$

então $\operatorname{Ext}^{1}\left(M_{i}, M_{j}\right)=0$.

Concluímos assim que cada $M_{i}$ é cotilting e $M_{i+1} \in^{\perp} M_{i}$, portanto a seqüência $\left\{M_{i}\right\}_{i \in \mathbb{N}}$ é uma seqüência cotilting em $\bmod B$. 


\subsection{Seqüências tiltings}

Analisaremos agora uma relação interessante entre duas seqüências tiltings em uma componente de conexão de uma álgebra inclinada $B$.

Definição 4.3.1 Sejam $R$ um anel, $T$ e $N$ dois A-módulos $n$-tiltings de tipo finito para algum $n \in \mathbb{N}$. Dizemos que $T$ e $N$ são Add-equivalentes se $\operatorname{Add} T=\operatorname{Add} N$.

Proposição 4.3.2 Sejam $\left\{T_{i}\right\}_{i \in \mathbb{N}} e\left\{H_{j}\right\}_{j \in \mathbb{N}}$ duas seqüências tiltings tais que:

a) Para cada $i \in \mathbb{N}$ existe um $j_{i}$ tal que $H_{j} \in T_{i}^{\perp}$ se $j>j_{i}$;

b) para cada $j \in \mathbb{N}$ existe $i_{j}$ tal que $T_{i} \in H_{j}^{\perp}$ para todo $i>i_{j}$;

Então $\left(\lim _{i \in \hat{\mathrm{N}}} T_{i}\right) e\left(\lim _{\overline{j \in \mathbb{N}}} N_{j}\right)$ são Add-equivalentes e, portanto geram tiltings maximais isomorfos.

Prova: Como sabemos que $\lim _{\bar{i} \in \mathrm{N}} T_{i}$ e $\lim _{\overline{j \in \mathrm{N}}} H_{j}$ são tiltings de tipo finito, basta mostrar que $\left(\lim _{i \in \mathbb{N}} T_{i}\right)^{\perp}=\left(\lim _{\bar{j} \in \mathbb{A}} H_{j}\right)^{\perp}$. De fato, para cada $T_{i}$ existe um índice $j_{i}$ tal que $\operatorname{Ext}^{m}\left(T_{i}, H_{j}\right)=0$ para todo $j \geq j_{i}$, então

$$
\operatorname{Ext}^{m}\left(T_{i}, \lim _{\overline{n \in \mathbb{N}}} N_{j}\right) \cong \lim _{i \in \mathbb{N}} \operatorname{Ext}^{m}\left(T_{i}, H_{j}\right)=0
$$

pelo Lema 1.5.10. Portanto $\lim _{\bar{j} \in \mathbb{N}} H_{j} \in T_{i}^{\perp}$ para todo $i \in \mathbb{N}$. Segue pelos Lemas 1.5.7 e 1.5.9 que $\operatorname{Ext}^{m}\left(\lim _{\bar{i} \in \hat{N}} T_{i}, \lim _{\overline{j \in \mathbb{N}}} H_{i}\right)=0$, ou seja $\lim _{\bar{j} \in \hat{N}} H_{j} \in\left(\lim _{i \in \mathbb{N}} T_{i}\right)^{\perp}$ e por 2.3.2 $\left(\lim _{\overline{j \in \mathbb{N}}} H_{j}\right)^{\perp} \subseteq\left(\lim _{i \in \mathbb{N}} T_{i}\right)^{\perp}$.

De forma análoga $\left(\lim _{i \in \mathbb{N}} T_{i}\right)^{\perp} \subseteq\left(\lim _{j \in \mathcal{N}} N_{j}\right)^{\perp}$. Concluímos então que

$$
\left(\lim _{\vec{i} \in \mathbb{N}} T_{i}\right)^{\perp}=\left(\lim _{\overrightarrow{j \in \mathbb{N}}} N_{j}\right)^{\perp},
$$

mas isso implica que

$$
\operatorname{Add}\left(\lim _{\bar{i} \in \mathbb{N}} T_{i}\right)=\operatorname{Add}\left(\lim _{\overline{j \in \hat{N}}} H_{j}\right)
$$


ou seja, $\lim _{i \in \mathbb{N}} T_{i}$ e $\lim _{\overline{j \in \mathbb{N}}} H_{j}$ são Add-equivalentes. Em outras palavras, esse módulos têm os mesmos somandos indecomponíveis. Isso nos diz que $\lim _{\bar{i} \in \mathbb{N}} T_{i}$ e $\lim _{\overline{j \in \mathbb{N}}} H_{j}$ geram módulos tiltings maximais isomorfos.

Lema 4.3.3 Sejam A uma álgebra hereditária, $T$ um A-módulo tilting clássico e $B=$ End $T$. Considere uma componente de conexão $\mathcal{C}$ de $\Gamma_{B}$ e $\Sigma$ o slice determinado por $T$ em $\mathcal{C}$. Suponha que $\mathcal{C}$ seja estável à direita. Sejam $T_{0}$ e $N_{0}$ dois módulos tiltings com todos os somandos indecomponíveis em $\Sigma^{+}$. Então existe um $n_{0} \in \mathbb{N}$ tal que $\tau^{-n_{0}} T_{0} \subseteq N_{0}^{\perp}$.

Prova: Seja $n_{0}$ tal que $\nu^{u}\left(N_{0}\right)<\nu_{d}\left(\tau^{-n_{0}-1} T_{0}\right)$, então $\operatorname{Hom}\left(\tau^{-n_{0}-1} T_{0}, N_{0}\right)=0$. Suponha por contradição que $\operatorname{Ext}^{1}\left(N_{0}, \tau^{-n_{0}} T_{0}\right) \neq 0$, então pela fórmula de Auslander Reiten 1.6.6 $\operatorname{Hom}\left(\tau^{-n_{0}-1} T_{0}, N_{0}\right) \neq 0$, o que é uma contradição. Portanto $\operatorname{Ext}^{1}\left(N_{0}, \tau^{-n_{0}} T_{0}\right)=0$ e como $\operatorname{pd} N_{0} \leq 1, \tau^{-n_{0}} T_{0} \in N_{0}^{\perp}$.

Naturalmente, repetindo o argumento do lema acima, existirá um $m_{0}>0$ tal que $\tau^{-m_{0}} N_{0} \subseteq T_{0}^{\perp}$. Isso nos diz que seqüências obtidas a partir de dois módulos tiltings em uma componente de conexão estável à direita de $\Gamma_{B}$ satisfazem as hipóteses da Proposição 4.3.2 e portanto os limites dessas seqüências serão Add-equivalentes.

Proposição 4.3.4 Sejam A uma álgebra hereditária, $T$ um A-módulo tilting clássico, $B=\operatorname{End} T$ e $\mathcal{C}$ uma componente de conexão de $\Gamma_{B}$. Suponha $\mathcal{C}$ estável à direita $e$ sejam $n \in \mathbb{N}$ e $M$ um módulo tilting com todos os somandos indecomponíveis $\Sigma^{+}$. Então para cada $n \in \mathbb{N} \quad \tau^{-n} M$ é um $B$-módulo tilting.

Prova: Como $M \in \operatorname{Add} \Sigma^{+}$, então cada somando de $M$ está em $\mathcal{C}$ e portanto cada somando de $\tau^{-n} M \in \mathcal{C}$, pois $\mathcal{C}$ é estável à direita. Disso segue que $\tau^{-n} M \in \operatorname{Add} \mathcal{C} \mathrm{e}$ $\operatorname{pd} \tau^{-n} M \leq 1$. Observe que o número de somandos de $\tau^{-n} M$ é exatamente o mesmo número de somandos de $M$. 
Suponha que $\operatorname{Ext}^{1}\left(\tau^{-n} M, \tau^{-n} M\right) \neq 0$, então $\operatorname{Hom}\left(\tau^{-n} M, \tau^{-n+1} M\right) \neq 0$. Se $\underline{\operatorname{Hom}}\left(\tau^{-n} M, \tau^{-n+1} M\right)=0$ existe um morfismo não nulo $f: \tau^{-n} M \longrightarrow P$ para algum projetivo indecomponível $P$. Como para $n>1$, todos os somandos de $\tau^{-n} M \in$ $\Sigma^{+}$, existirá um morfismo não nulo de $\mathcal{X}(T)$ para $\mathcal{Y}(T)$, pois qualquer $B$-módulo projetivo indecomponível pertence a $\mathcal{Y}(T)$, mas isso é uma contradição. Portanto $\underline{\operatorname{Hom}}\left(\tau^{-n} M, \tau^{-n+1} M\right) \neq 0$. Segue pela equivalência $\tau: \underline{\bmod } B \longrightarrow \overline{\bmod } B$ que

$$
\operatorname{Hom}\left(\tau^{-n+1} M, \tau^{-n+2} M\right) \neq 0 \text {. }
$$

Repetindo o argumento para $\operatorname{Hom}\left(\tau^{-n+i} M, \tau^{-n+i+1} M\right)$ enquanto $0<i \leq n-1$, temos ao fim do processo, $\underline{\operatorname{Hom}}\left(\tau^{-} M, M\right) \neq 0$. Mas nesse caso $\operatorname{Ext}^{1}(M, M) \neq 0$, o que é uma contradição. Portanto $\operatorname{Ext}^{i}\left(\tau^{-n} M, \tau^{-n} M\right)=0$. Logo $\tau^{-n} M$ é tilting.

Iremos agora obter uma seqüência tilting a partir dos transladados de um $B$ módulo tilting $M$ em $\operatorname{Add} \Sigma^{+}$.

Dizemos que uma seqüência $\left\{T_{i}\right\}_{i \in \mathbb{N}}$ de módulos tiltings em uma componente de conexão estável à direita é crescente se existir um $m_{0} \in \mathbb{N}$ tal que $\nu^{u}\left(T_{i}\right)<\nu^{u}\left(T_{j}\right)$ e $\nu^{u}\left(T_{i}\right) \leq \nu_{d}\left(T_{j}\right)+m_{0}$ para todo $i \leq j$.

Proposição 4.3.5 Sejam $\mathcal{C}$ uma componente de conexão estável à direita $e M$ um módulo tilting com todos os somandos em $\Sigma^{+}$. Então existe $m_{0} \in \mathbb{N}$ tal que os módulos da forma $T_{i}=\tau^{-i m_{0}} M$ constituem uma seqüência tilting crescente $\left\{T_{i}\right\}_{i \in \mathbb{N}}$.

Prova: Seja $m_{0}$ tal que $\nu^{u}(M)<\nu_{d}\left(\tau^{-m_{0}-1} M\right)$, então pela Proposição 4.2.11 $\operatorname{Hom}\left(\tau^{-m_{0}-1} M, M\right)=0$. Segue pela fórmula de Auslander Reiten 1.6.6 que

$$
\operatorname{Ext}^{1}\left(M, \tau^{-m_{0}} M\right)=0,
$$

portanto $\tau^{-m_{0}} M \in M^{\perp}$. Analogamente para qualquer $i>0$ temos que $\nu^{u}\left(\tau^{-i m_{0}} M\right)<$ $\nu_{d}\left(\tau^{-(i+1) m_{0}-1} M\right)$. Então $\operatorname{Ext}^{1}\left(\tau^{-i m_{0}} M, \tau^{-(i+1) m_{0}} M\right)=0$ pelo mesmo argumento utilizado acima. Logo $T_{i+1} \in T_{i}^{\perp}$. Como cada $T_{i}=\tau^{-i m_{0}} M$ é tilting pela Proposição 4.3.4 e $M$ é tilting por hipótese, a seqüência de módulos $\left\{T_{i}\right\}_{i \in \mathbb{N}}$ forma uma 
seqüência tilting em $\bmod B$. A seqüência é obviamente crescente pela forma com que foi construída.

Para finalizar veremos agora que álgebras hereditárias e álgebras concealed de tipo de representação infinito admitem seqüências tiltings, e portanto é possível obter módulos tiltings não finitamente gerados sobre essa álgebras.

Corolário 4.3.6 Se B é uma álgebra hereditária básica indecomponivel de tipo de representação infinito, ou se $B$ é concealed, então existem módulos tiltings e cotiltings infinitos em $\operatorname{Mod} B$.

Prova: Basta observar que a componente pós-projetiva de $B$ é uma componente de conexão estável à direita e que a componente pré-injetiva é também uma componente de conexão e é estável à esquerda. O resultado agora segue pelo Teorema 4.2.12.

Apresentamos o exemplo abaixo para mostrar que se no Teorema 3.2.1 não exigirmos que $\operatorname{Add} T_{i} \neq \operatorname{Add} T_{j}$ e não exigirmos seqüências tiltings crescentes então podemos obter seqüências tiltings "constantes" e com limites tendo somandos indecomponíveis distintos.

Exemplo: Sejam $A$ uma $\mathbb{K}$-Álgebra, $n \in \mathbb{N}, T$ e $N$ dois $A$-módulos $n$-tilting tais que $\operatorname{Add} T \neq \operatorname{Add} N$ e seqüências: $\left\{T_{i}\right\}_{i \in \mathbb{N}} \operatorname{com} T_{i}=T$ e $\left\{N_{j}\right\}_{j \in \mathbb{N}} \operatorname{com} N_{j}=N$. Então $\lim _{i \in \vec{N}} T_{i}=T$ e $\lim _{i \in \vec{N}} N_{j}=N, \operatorname{mas} \operatorname{Add} T \neq \operatorname{Add} N$.

Veja nas figuras 4.1 e 4.2 exemplos de configuração dos módulos nas componentes de conexão de uma álgebra hereditária. Essas figuras permitem visualizar como podemos conseguir as seqüências tiltings ou cotiltings em sobre essa componentes. $\mathrm{Na}$ figura 4.1 partimos da seção definida pelos projetivos na componente pós-projetiva e construímos uma seqüência tilting tomado os transladados de Auslander Reiten $\tau^{-n}$ dos módulos nessa seção. Na figura 4.2 tomamos a seção formada pelos módulos injetivos e construímos uma seqüência cotilting através dos transladados $\tau^{n}$. 


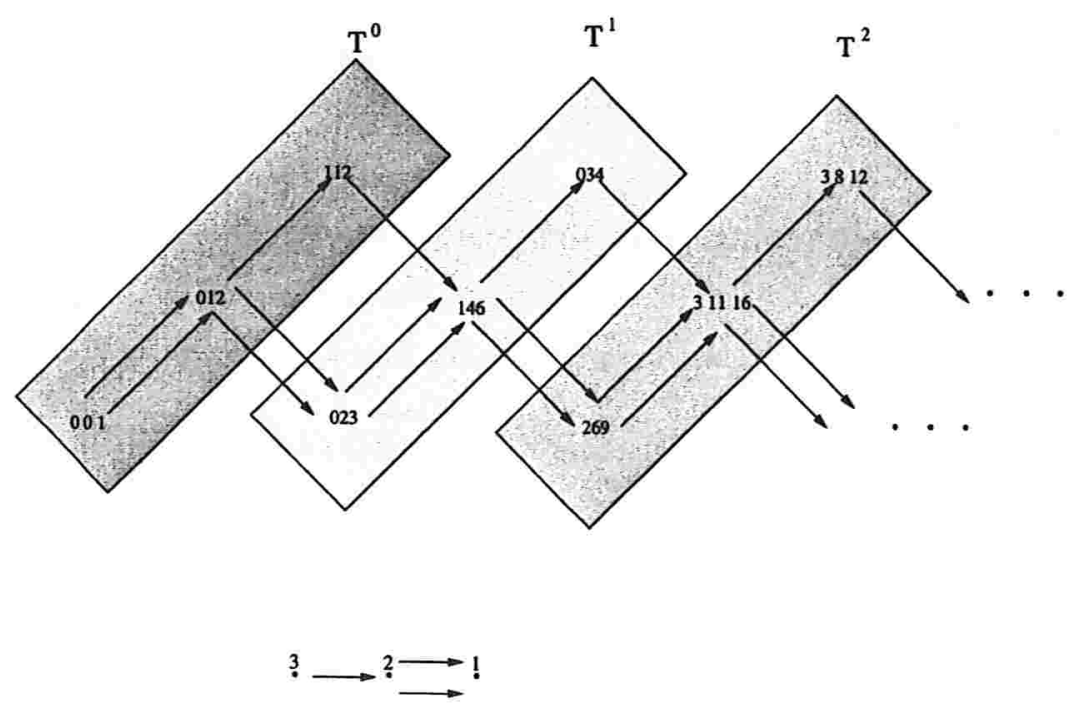

Figura 4.1: Seqüência tilting em uma componente de conexão estável à direita

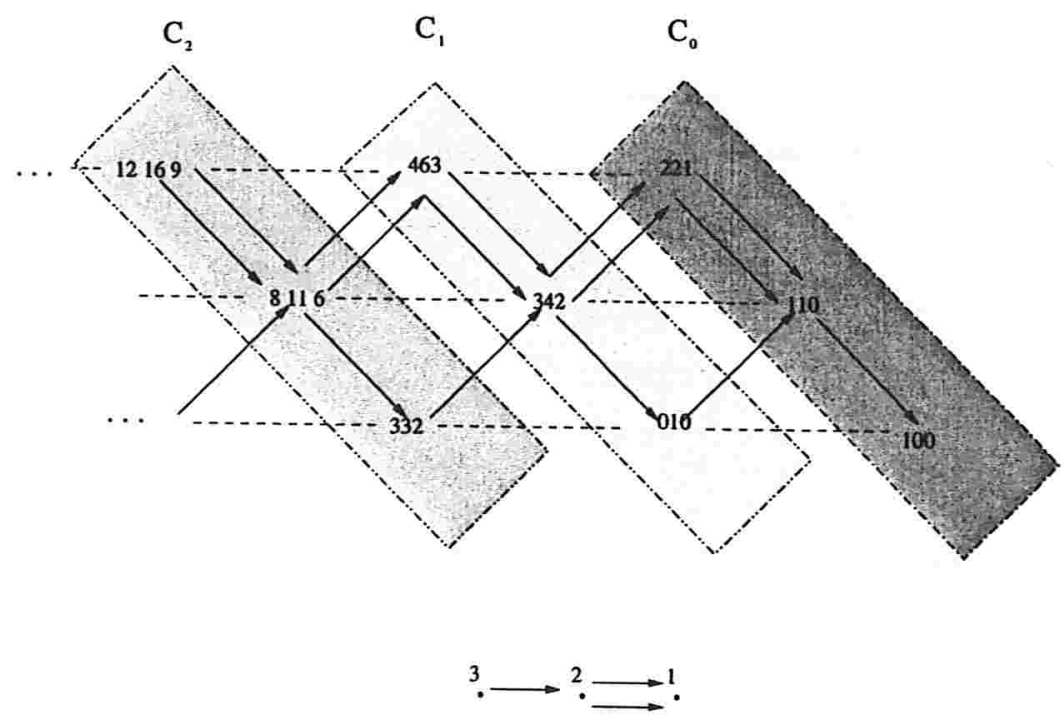

Figura 4.2: Seqüência cotilting em uma componente de conexão estável à esquerda 


\subsection{Módulos fg-colimites}

Apresentaremos nessa seção uma condição necessária à existência de uma certa classe de módulos t-colimites sobre álgebras inclinadas.

Vamos supor que todas as álgebras trabalhadas durante a seção sejam $\mathbb{K}$-álgebras sobre um corpo algebricamente fechado $\mathbb{K}$.

Sejam $A$ uma $\mathbb{K}$ - álgebra e $T \in \operatorname{Mod} A$ um módulo $n$-tilting, para algum natural $n$. Dizemos que $T$ é um tilting fg-colimite se existir uma família de módulos tiltings clássicos $\left\{T_{i}\right\}_{i \in \mathbb{N}}$ em $\bmod A$ tais que $\operatorname{Add} T_{i} \neq \operatorname{Add} T_{j}$ se $i \neq j, T_{i+1} \in\left(T_{i}\right)^{\perp}$ e $T=\lim _{i \in \mathbb{N}} T_{i}$. Analogamente um módulo é dito fg-limite se existe uma família de módulos cotiltings $C_{i} \in \mathbb{N}$ com dimensão projetiva inferior a 1 .

Pelo Teorema 3.2.1 se $T=\lim _{i \in \mathbb{N}} T_{i}$, então $\operatorname{pd} T \leq 2$ e portanto $T$ é 2-tilting. Sabemos também que se $B$ for uma álgebra inclinada que possui uma componente de conexão estável à direita, então existem módulos tiltings fg-colimites em $\bmod B$.

Poderíamos perguntar então se toda álgebra inclinada admite um módulo tilting fg-colimite. Nesse sentido, observa-se, em primeiro lugar, que em geral se existir um tal módulo, então existem infinitos módulos indecomponíveis finitamente gerados com dimensão projetiva 1 . Em particular $B$ tem que ser de tipo de representação infinito.

Exibiremos a seguir uma condição necessária para a existência de módulos fgcolimites em álgebras inclinadas. Para tanto vamos necessitar de uma série de novos conceitos. Seguiremos a ordem de apresentação e as definições de [3] para apresentar a noção de tipos reduzidos à direita e à esquerda de uma álgebra, bem como algumas proposições que serão usadas aqui.

Seja $B$ uma $\mathbb{K}$-álgebra inclinada conexa, então existe um quiver finito conexo e sem ciclos orientados $\Sigma$ e um módulo tilting $T$ sobre a $\mathbb{K}$-álgebra $\mathbb{K} \Sigma$ tais que $B=$ End $T$. Então o quiver de Auslander Reiten de $B$ possui um slice cujo quiver é isomorfo a $\Sigma^{o p}$. Esse quiver, em geral não é unicamente determinado por $B$, mas se

$\Sigma$ e $\Sigma^{\prime}$ são quivers obtidos da forma acima, então eles têm o mesmo grafo subjacente e podem ser obtidos um do outro por uma seqüência admissível de reflexões. O grafo 
subjacente $\bar{\Sigma}$ do quiver $\Sigma$ é denominado o tipo de $B$.

Definiremos o tipo à esquerda de $B$ da seguinte forma. Se $B$ possui um slice em uma componente pós-projetiva, o tipo à esquerda de $B$ é o grafo vazio. Se isso não ocorre, então $B$ possui uma única componente de conexão $\mathcal{C}$ que não é pós-projetiva. Se $\mathcal{C}$ não possui módulos projetivos, isto é, se $\mathcal{C}$ é estável à esquerda, então o tipo à esquerda de $B$ é o tipo de $B$. Suponha então que $\mathcal{C}$ possua módulos projetivos. Seja $\Sigma$ a subseção de $\mathcal{C}$ constituída pelos módulos estáveis à esquerda $M$ em $\mathcal{C}$ tais que existe um caminho em $\mathcal{C}$ de comprimento maior ou igual a 1 de $M$ para algum projetivo e tal que qualquer possível caminho entre $M$ e um projetivo seja seccional. A subseção $\Sigma$ em geral não é conexa, então $\Sigma=\Sigma_{1} \cup \Sigma_{2} \cup \ldots \cup \Sigma_{t}$, onde cada $\Sigma_{i}$ é uma das componente conexas de $\Sigma$. Chamaremos $\Sigma$ de subseção extrema à esquerda de $B$. O grafo subjacente a $\Sigma$ será chamado de tipo à esquerda de $B$.

Dualmente definiremos o tipo à direita de $B$ como segue. Se $B$ possui uma componente pré-injetiva, então o tipo à direita de $A$ é definido como sendo o grafo vazio. Caso contrário $B$ possui uma única componente de conexão $\mathcal{C}$ a qual não é pré-injetiva. Se $\mathcal{C}$ não possui módulos injetivos, então definimos o tipo à direita de $B$ como sendo o próprio tipo de $B$. Se $\mathcal{C}$ contém módulo injetivos, seja $\Sigma$ a subseção de $\mathcal{C}$ consistindo de todos os módulos estáveis à direita $M \in \mathcal{C}$ tais que exista um caminho de comprimento maior o igual a 1 de um injetivo para $M$ e qualquer desses caminhos seja seccional. Seja $\Sigma=\Sigma_{1} \cup \Sigma_{2} \cup \Sigma_{3} \cup \ldots \cup \Sigma_{t}$, onde cada $\Sigma_{i}$ é uma componente conexa de $\Sigma$. A subseção $\Sigma$ será chamada de subseção extrema à direita de $B$ e o grafo subjacente será denominado de tipo à direita de $B$.

Proposição 4.4.1 Seja B uma álgebra inclinada de tipo de representação infinito. $B$ é concealed se e somente se $\operatorname{pd} M=1$ e $\mathrm{id} M=1$ para quase todos os módulos, isto é, existe apenas um número finito de módulos para os quais $\operatorname{pd} M \neq 1$ ou $\operatorname{id} M \neq 1$.

Prova: Veja [3] Proposição 3.3.

Seja $B$ uma álgebra inclinada de tipo de representação infinito. Se $B$ tem uma componente pós-projetiva contendo um slice, ou se a única componente de conexão 
de $B$ não contém módulo projetivos, definimos tipo reduzido à esquerda. de $B$ como sendo o tipo à esquerda de $A$. Assuma que a única componente de conexão de $B$ não é pós-projetiva, mas contém projetivos, e seja $\Sigma=\Sigma_{1} \cup \Sigma_{2} \cup \Sigma_{3} \cup \ldots \cup \Sigma_{t}$ a subseção extrema à esquerda de $B$. Definimos a subseção extrema reduzida à esquerda de $B$ como sendo $\Sigma_{1}^{\prime} \cup \Sigma_{2}^{\prime} \cup \Sigma_{3}^{\prime} \cup \ldots \cup \Sigma_{t}^{\prime}$, onde para cada $i, \Sigma_{i}^{\prime}$ é o subquiver pleno de $\Sigma_{i}$ obtido removendo-se todos os vértices poços. $\mathrm{O}$ tipo reduzido de $B$ é o grafo subjacente à subseção extrema à esquerda reduzida de $B$.

De forma análoga definimos o tipo reduzido à direita de $B$. Se $B$ possui uma componente pré-injetiva contendo um slice ou se a única componente de conexão de $B$ não contém módulos injetivos, definimos o tipo reduzido à direita de $A$ como sendo o tipo à direta de $A$. Assuma que a única componente de conexão de $B$ não seja pré-injetiva mas contenha injetivos, e seja $\Sigma=\Sigma_{1} \cup \Sigma_{2} \cup \Sigma_{3} \cup \ldots \cup \Sigma_{t}$ uma subseção extrema à direta de $B$. Definiremos o a subseção extrema reduzida à direita de $A$ como sendo $\Sigma_{1}^{\prime} \cup \Sigma_{2}^{\prime} \cup \Sigma_{3}^{\prime} \cup \ldots \cup \Sigma_{t}^{\prime}$, onde para cada $i, \Sigma_{i}^{\prime}$ é o subquiver pleno de $\Sigma_{i}$ obtido removendo-se todos os vértices fontes. $\mathrm{O}$ tipo reduzido à direita de $B$ será definido como sendo o grafo subjacente à $\Sigma$. A proposição abaixo e o corolário que a segue são devidas a Assem e Coelho e recomendamos uma leitura de [3] para os detalhes da demonstração.

Proposição 4.4.2 Seja B uma álgebra inclinada de tipo de representação infinita que não seja concealed, e $\Sigma$ um slice em $\Gamma_{B}$.

a) $\operatorname{pd} M=2$ para quase todos os sucessores indecomponiveis $M$ de $\Sigma$ se e somente se o tipo reduzido a direta de $B$ é vazio ou uma união disjunta de grafos Dynkin.

b) $\operatorname{id} M=2$ para quase todos os predecessores indecomponiveis $M$ de $\Sigma$ se e somente se o tipo reduzido à esquerda de $B$ é vazio ou uma união disjunta de grafos Dynkin.

Corolário 4.4.3 Seja $B$ uma álgebra inclinada de tipo de representação infinita. 
a) Se A tem um slice em uma componente pós-projetiva, então $\operatorname{pd} M=2$ para quase todos os módulos indecomponiveis $M$ se e somente se o tipo reduzido à direta de $B$ é uma união disjunta de grafos Dynkin.

b) Se A tem um slice completo em uma componente pré-injetiva, então id $M=2$ para quase todos os módulos indecomponiveis $M$ se e somente se o tipo reduzido à esquerda de A for uma união disjunta de grafos Dynkin.

Vamos agora a proposição que fornece uma condição necessária à existência de módulos fg-colimites em álgebras inclinadas

Proposição 4.4.4 Seja B uma álgebra inclinada de tipo de representação infinito que não seja concealed e $\Sigma$ o slice na componente de conexão $\mathcal{C}$ de de $\Gamma_{B}$.

a) $\mathrm{Se} \mathcal{C}$ for pós-projetiva e $A$ admite um módulo tilting fg-colimite, então seu tipo reduzido à direita possui um subgrafo euclidiano.

b) $S e \mathcal{C}$ for pré-injetiva e $A$ admite um módulo tilting fg-colimite, então seu tipo reduzido à direita possui um subgrafo euclidiano.

Prova: Faremos somente a prova de a) sendo que b) á dual. Como existe pelo menos um módulo fg-limite em $\bmod B$, existirá infinitos módulos tiltings finitamente gerados com dimensão projetiva menor ou igual a 1. Segue pelo Corolário 4.4.3 a) que o tipo reduzido á direta de $B$ possui um subgrafo não Dynkin e conseqüentemente possui um subgrafo euclidiano.

Pode não existir uma componente de conexão pós-projetiva, ou uma componente pré injetiva, mas o resultado é válido caso exista.

A contrapositiva da proposição acima permite-nos afirmar que se $B$ for uma álgebra inclinada de tipo de representação infinito que não seja concealed e $\Sigma$ um slice na componente pós-projetiva de $\Gamma_{B}$, se o tipo reduzido à direita de $B$ é uma união de grafos Dynkin, então $B$ não admite um módulo fg-colimite. 


\subsection{Outras álgebras com módulos t-colimites}

Nesse seção veremos como obter módulos t-colimites em outro tipo álgebras, as que possuem componentes pós-projetivas standard generalizadas e estáveis à direita.

Definição 4.5.1 Sejam A uma $\mathbb{K}$-álgebra e $\mathcal{C}$ uma componente do quiver de Auslander Reiten $\Gamma_{A}$ de A. Dizemos que $\mathcal{C}$ é standard generalizada se $\operatorname{rad}^{\infty}(X, Y)=0$ para qualquer $X, Y \in \mathcal{C}$.

Lema 4.5.2 Seja A uma álgebra básica indecomponivel contendo uma componente pós-projetiva $\mathcal{C}$ e escreva $A \cong P_{1} \amalg P_{2}$. Suponha que $P_{1} \in$ add $\mathcal{C}$, nenhum somando de $P_{2}$ pertence a $\mathcal{C}$ e que $\operatorname{Hom}\left(P_{2}, P_{1}\right)=0$. Então annC $\cong P_{2}$.

Prova: Seja $A=A e_{1} \amalg A e_{2}$ uma decomposição do $A$-módulo regular $A$ tal que $A e_{1} \cong P_{1}$ e $A e_{2} \cong P_{2}$. Considere $x \in$ annC, então $x P_{1}=0$, logo $x A e_{1}=0$. Sendo $x=x e_{1}+x e_{2}$ e $x e_{1} \in A e_{1}$, então $x=x e_{2}, \operatorname{logo} x \in A e_{2}$. Portanto annC $\subseteq A e_{2}$. Considere agora $x \in A e_{2}$, então $x=\lambda e_{2}$ para algum $\lambda \in A$. Como $e_{2} A e_{1} \cong$ $\operatorname{Hom}\left(A e_{2}, A e_{1}\right) \cong \operatorname{Hom}\left(P_{2}, P_{1}\right)=0$ temos que $\lambda e_{2} P_{1}=0$. Portanto $\lambda e_{2} \in \operatorname{ann} P_{1}$. Como os somandos de $P_{1}$ formam uma seção de $\mathcal{C}$, então ann $P_{1}=$ annC por [31]. Portanto $x \in$ ann $\mathcal{C}$ e conseqüentemente annC $=A e_{2}$. Logo annC $\cong P_{2}$.

Teorema 4.5.3 Sejam A uma $\mathbb{K}$-álgebra de dimensão finita contendo uma componente pós-projetiva $\mathcal{C}$ e $B=\frac{A}{\operatorname{ann} \mathcal{C}}$. Suponhamos que $\mathcal{C}$ seja standard generalizada, estável à direita e contendo uma seção finita $\Delta$. Então existe um módulo $t$-colimite em $A$.

Prova: Seja $B=\frac{A}{\operatorname{ann\mathcal {C}}}$. Sabemos por [31] que $B$ é inclinada e $\mathcal{C}$ é a componente de conexão de $\Gamma_{B}$. Então os módulos de $\Delta$ formam um slice em $\Gamma_{B}$ e $M=\coprod_{M_{i} \in \Delta_{0}} M_{i}$ é um $B$-módulo tilting. Como $\mathcal{C}$ é estável à direita $T_{i}=\tau^{-i} M$ forma uma seqüencia tilting em $\Gamma_{B}$, pois para um A-módulo $M$ com todos os somandos em $\mathcal{C}$ ser $B$-módulo é equivalente a ser $A$-módulo, portanto $\operatorname{Ext}\left(T_{i}, T_{j}\right)=0$ se $i<j$ e $\operatorname{pd}_{A}\left(T_{k}\right) \leq 1$ para 
todo $k \in \mathbb{N}$, pois todos os $B$-módulos projetivos indecomponíveis estão em $\mathcal{C}$ e são também projetivos em $A$. Logo $\left\{T_{i}\right\}_{i \in \mathbb{N}}$ formam uma seqüência tilting em $\bmod B$. Então cada $T_{i}$ é um $A$-módulo tilting parcial, portanto pela Proposição 2.4.2 cada $T_{i}$ pode ser completado a um $A$-módulo tilting digamos $T_{i} \amalg M_{i}=H_{i} \operatorname{com} H_{i}^{\perp}=T_{i}^{\perp}$ em $\operatorname{Mod} A$, Então $\operatorname{pd} H_{i} \leq \operatorname{pd} T_{i}<n$. Logo $\left\{H_{i}\right\}$ é uma seqüencia tilting em $\operatorname{Mod} A$. $\mathrm{O}$ resultado agora segue pelo Teorema $\mathbf{3 . 2 . 1}$.

A prova que apresentamos acima utiliza do teorema de completamento 2.4.2 para completar cada módulo tilting parcial e portanto sabemos que existe o complemento mas não sabemos quem é esse módulo. Vamos sugerir uma outra prova que deixará mais explícito onde podemos encontrar um complemento para esses módulos.

Outra prova: Seja $B=\frac{A}{\operatorname{ann\mathcal {C}}}$. Sabemos por [31] que $B$ é inclinada e $\mathcal{C}$ é a componente de conexão de $\Gamma_{B}$. Então os módulos de $\Delta$ constituem um slice em $\Gamma_{B}$ e $M=\coprod_{M_{i} \in \Delta_{0}} M_{i}$ é um $B$-módulo tilting. Como $\mathcal{C}$ é estável à direita $T_{i}=\tau^{-i} M$ forma uma seqüência tilting em $\Gamma_{B}$, pois um A-módulo $M$ com todos os somandos em $\mathcal{C}$ é $B$-módulo e todo $B$-módulos de $\mathcal{C}$ é também um $A$-módulo, portanto $\operatorname{Ext}\left(T_{i}, T_{j}\right)=0$ se $i<j$ e $\operatorname{pd}_{A}\left(T_{k}\right) \leq 1$ para todo $k \in \mathbb{N}$, pois todos os $B$-módulos projetivos indecomponíveis estão em $\mathcal{C}$ e são também projetivos em $A$. Logo $\left\{T_{i}\right\}_{i \in \mathbb{N}}$ é uma seqüência tilting em $\bmod B$. Como cada $T_{i}$ é um $B$-módulo tilting, temos um diagrama

$$
0 \longrightarrow B \longrightarrow T_{i}^{0} \longrightarrow T_{i}^{1} \longrightarrow 0
$$

para cada $i \in \mathbb{N} \operatorname{com} T_{i}^{0}$ e $T_{i}^{1} \in \operatorname{add} T_{i}$. Olhando em $\bmod A$, temos que $A \cong B \amalg P$, onde os somandos de $P$ não pertencem a $\mathcal{C}$. Logo obtemos a seqüência

$$
0 \longrightarrow A \longrightarrow T_{i}^{0} \coprod P \longrightarrow T_{i}^{1} \longrightarrow 0
$$

que é uma seqüencia exata em $\bmod A \operatorname{com} T_{i}^{0} \amalg P$ e $T_{i}^{1} \in \operatorname{add}\left(T_{i}^{0} \amalg P\right)$. Portanto $T_{i} \amalg P$ é um módulo tilting. Nesse caso temos uma seqüência tilting $\left\{T_{i} \amalg P\right\}_{i \in \mathbb{N}}$ em $\bmod A$ e o resultado segue pelo Teorema 3.2.1. Portanto existe um módulo 
$t$-colimite $\operatorname{em} \operatorname{Mod} A$.

Observe que $H_{i}^{\perp}=T_{i}^{\perp}=T_{i} \amalg P$ e portanto $\operatorname{Add} H_{i}=\operatorname{Add}\left(T_{i} \amalg P\right)$. Então os módulos $H_{i}$ e $T_{i}$ são Add-equivalentes e não faz diferença o modo com que completamos os módulos tiltings parciais que sempre teremos limites Add-equivalentes.

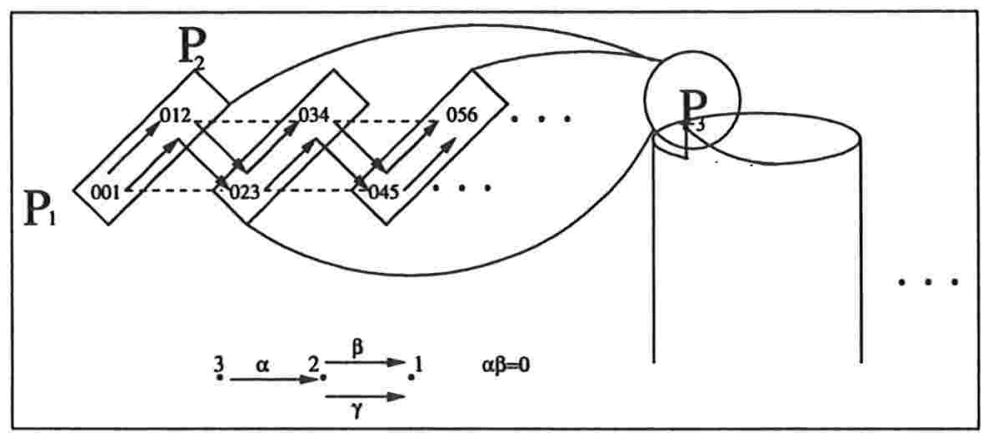

Figura 4.3: Seqüência tilting obtida em uma componente standard generalizada estável à direita

Na figura 4.3 mostramos um exemplo de como construir seqüência tilting em componentes pós-projetivas que satisfaçam a hipóteses do Teorema 4.5 .3 e cuja álgebra satisfaçam as hipóteses do Lema 4.5.2. 


\section{Referências Bibliográficas}

[1] Assem I., Tilting Theory - An introduction, In Topics in algebra, Banach Center Publication 26 (1990), 127-180.

[2] Auslander M. and Buchweitz R., The homological theory of maximal CohenMacaulay approximations, Soc Math de France, Mémoire, 38 (1989), 15-37.

[3] Assem I. and Coelho F., Complete slices and homological properties of tilted algebras, Glasgow Math J. 36 (1994), 347-354.

[4] Assem I., Simson D. and Skowroński Elements of Representation Theory of Associative Algebras, London Mathematical Society Student Texts 65.

[5] Anderson F. Fuller K., Ring and categories of modules, Graduate Texts in Mathematics 13 Springer (1991).

[6] Angeleri-Hügel L. and Coelho F., Infinitely generated tilting modules of finite projective dimension, Forum Math., 13 (2001) n. 2, 239-250.

[7] Angeleri-Hügel L. and Coelho F., Infinitely generated complements to partial tilting modules, Math. Proc. Cambridge Phil. Soc. 132 (2002) n 1, 89-96.

[8] Angeleri-Hügel L., Herbera D. and Trlifaj J., Tilting modules and Gorenstein Rings, to appear in Forum Mathematicum.

[9] Angeleri-Hügel L., Tonolo A. and Trlifaj J., Tilting preenvelops and cotilting precovers, Math Pura ed Appl. Universidade de Pandova 
[10] Angeleri-Hügel L. and Trlifaj J., Tilting theory and the finitistic dimension conjectures,Trans. Amer. Math. Soc. 354 (2002), 4345-4358.

[11] Angeleri-Hügel L., Tonolo L. and Trlifaj J., Tilting preenvelopes and cotilting precovers, Algebras and Repres. Theory 4 (2001), 155-170.

[12] Auslander M. and Reiten I., Applications of contravariantly finite subcategories, Adv. Math. 86 (1991), 111-152.

[13] Auslander M., Reiten I. and SmaløS., Representation theory of artin algebras, Cambridge Studies in Advanced Mathematics 36 (1995), Cambridge Univ. Press.

[14] Auslander M. and Smalø S., Preprojective modules over artin algebras, Journal of Algebra 66 (1980), 61-122.

[15] Auslander M. and Smalø S., Almost split sequences in subcategries, Journal of Algebra 69 (1981), 426-454; Addendum J. Algebra 71 (1981), 592-594 .

[16] Bongartz K., Tilted Algebras, Proc. ICRA III (Puebla) in Lectures Notes in Math. 903 Springer-Verlag (1981), 26-38.

[17] Brenner S. and Butler M., Generalization of the Bernstein-Gelfand-Panomarev reflections functor, Proc. of ICRA II Spring Lectures Notes in Mathematics 832 (1980), 103-169.

[18] Buan A. and Solberg O., Systems and limits of finitely generated cotilting modules, to appear.

[19] Buan A. and Solberg O., Limites of Pure-Injective Cotilting Modules, Journal of algebras and Representations Theory, to appear

[20] Buan A., Relative cotilting theory and partial cotilting modules, to appear.

[21] Coelho F., Happel D. and Unger L., Complements to partial tilting modules, Journal of Algebra, 170 (1994), 184-205. 
[22] Colpi R. and Trlifaj J., Tilting modules and tilting torsion theories, J. Algebra 178 (1995), 614-634.

[23] Enochs., Injetive a flat cover, envelopes a resolvents, Israel J. Math. 39 (1981), 189-209.

[24] Enochs E. and Jenda O., Relative Homological Algebra, De Gruyter Expositions in Mathematics 30.

[25] Eklof P. and Trlifaj J., How to make Ext Vanish, Bull. London Math. Soc. 33 (2001), 41-51.

[26] Faith C., Algebra II - Ring Theory, Springer Verlag (1976).

[27] Fuller K. and Saorin M., On the finitistic dimension conjecture for artinian rings, Man. Math. 74 (1992), 117-132.

[28] Gabriel P., Unzerlegbare Darstellugen I, Manuscripta Math. 6 (1972), 71-103

[29] Happel D. and Ringel C., Tilted algebras, Trans. Amer. Math. Soc. 274 (1982), 399-443.

[30] Jensen C. and Lenzing H., Model-Theorectic algebra with particular emphasis on fields, rings, modules, Algebra, Logic and applications 2, Gordon and Breach Science Publication, New York (1989).

[31] Liu X., Tilted algebras and generalized standard Auslander-Reiten components, Arch Math. 12 (1993), 12-19.

[32] Miyashita Y., Tilting modules of finite projective dimension, Math Z. 193 (1986), 113-146.

[33] Rickard J. and Schofield A., Cocovers and Tilting modules, Math. Proceedings of the Cambridge Philosophical Societ. 106 (1989), 1-5. 
[34] Ringel C. M., Tame algebras e integral quadratic forms, Lectures Notes in Mathematics 1099 Springer (1984).

[35] Ringel C. M., The regular componetes of the Auslander-Reiten quiver of a tilted algebra, Chinese Ann. Math. Ser. B 9 (1988), 1-18.

[36] Rotman J., An Introduction to Homological Algebra, Academic Press.

[37] Salce L., Cotorsion theories for abelian groups, Symposia Math. XXIII (1979), 11-32.

[38] Xu J., Flat covers of Modules, Lectures Notes in Mathematics 1634 (1991). 


\title{
Índice Remissivo
}

\author{
álgebra, 6 \\ concealed, 69 \\ de dimensão finita, 6 \\ hereditária, 63 \\ inclinada, 67 \\ índice de translação, 74 \\ Add-equivalentes, 79 \\ anel \\ coerente, 17 \\ caminho \\ seccional, 73 \\ categoria, 8 \\ categorias \\ dualidade de, 10 \\ equivalência de, 10 \\ classe \\ $n$-cotilting, 30 \\ pré-envolvente, 14 \\ recobrimento, 14 \\ classes \\ $n$-tilting, 29 \\ $n$-tilting de tipo finito, 29 \\ s-fechadas, 13 \\ classes c-fechadas, 13 \\ componente \\ estável \\ à direita, 74 \\ à esquerda, 74 \\ pós-projetiva, 26 \\ pré-injetiva, 26 \\ standard generalizada, 88 \\ conjunto \\ quase ordenado, 15 \\ conjunto dirigido, 15 \\ cotorção \\ par \\ completo, 14 \\ de tipo finito, 14 \\ par de, 14 \\ fechada \\ para predecessores, 68 \\ para sucessores, 68 \\ fg-colimite, 84 \\ Five Lemma, 44 \\ funtor, 9 \\ contravariante, 9 \\ covariante, 9
}


funtores

isomorfos, 10

quase inversos, 10

lema

Dimension shifting, 20

Lema do cinco, 44

módulo

ct-limite, 62

$n$-cotilting, 29

$n$-tilting, 29

$t$-colimite, 62

fiel, 29

sincero, 29

tilting parcial, 29

morfismo

irredutível, 25

minimal à esquerda, 24

minimal quase cindido à direita, 25

minimal quase cindido à esquerda, 25

quase cindido à direita, 24

quase cindido à esquerda, 24

morfismo retração, 24

morfismo seção, 24

objetos, 9

par de torção, 68

splitting, 70

pré-cobertura, 14 especial, 14

pré-envolvente, 13

especial, 14

quiver, 7

acíclico, 7

conexo, 7

finito, 7

seção, 72

seqüência

de Auslander Reiten, 25

quase cindida, 25

crescente, 81

seqüencia exata

de conexão, 69

sistema direto, 15

contínuo, 18

slice, 73

subcategoria, 9

plena, 9

subquiver, 7

pleno, 7

subseção, 73

subseção extrema reduzida, 86

T-corresolução, 37

Teorema

Brenner-Butler, 69

tilting

clássico, 67

maximal, 51 
tipo

à direita de uma álgebra, 85

à esquerda de uma álgebra, 85

de uma álgebra, 85

reduzido à direita, 86

reduzido à esquerda, 86

transformação natural, 9 